\title{
Knot Floer homology of Whitehead doubles
}

\author{
MatThew HedDEN
}

In this paper we study the knot Floer homology invariants of the twisted and untwisted Whitehead doubles of an arbitrary knot, $K$. A formula is presented for the filtered chain homotopy type of $\widehat{H F K}\left(D_{ \pm}(K, t)\right)$ in terms of the invariants for $K$, where $D_{ \pm}(K, t)$ denotes the $t$-twisted positive (resp. negative)-clasped Whitehead double of $K$. In particular, the formula can be used iteratively and can be used to compute the Floer homology of manifolds obtained by surgery on Whitehead doubles. An immediate corollary is that $\tau\left(D_{+}(K, t)\right)=1$ if $t<2 \tau(K)$ and zero otherwise, where $\tau$ is the Ozsváth-Szabó concordance invariant. It follows that the iterated untwisted Whitehead doubles of a knot satisfying $\tau(K)>0$ are not smoothly slice. Another corollary is a closed formula for the Floer homology of the three-manifold obtained by gluing the complement of an arbitrary knot, $K$, to the complement of the trefoil.

57M27; 57R58

\section{Introduction}

Satellite knots are frequently studied objects in the world of low-dimensional topology. Among the most famous satellite knots are the Whitehead doubles, which have been at the heart of many beautiful constructions (Akbulut-Matveyev [1;2], Freedman-Quinn [8] and Cochran-Gompf [4]). As discussed below, the untwisted double of an arbitrary knot has classical invariants such as the Alexander polynomial and signature identical to those of the unknot. Thus computing values for Whitehead doubles provides an interesting test of any new knot invariant's strength. Perhaps the Whitehead doubles have shone most brightly in the study of knot concordance, where they provide examples of knots which are topologically slice yet not smoothly slice. In this way the Whitehead doubles showcase the remarkable distinction between the smooth and topological categories in dimension four.

In recent years Ozsváth and Szabó have constructed a comprehensive and powerful set of invariants for low-dimensional topological and geometric objects using the Floer homology theory of pseudo-holomorphic curves. The purpose of this paper is to study the knot invariants introduced in Ozsváth-Szabó [27] and Rasmussen [33] in the context of Whitehead doubling. Our motivation is twofold: (1) to obtain a better 
understanding of the $(2+1)$ dimensional topological quantum field theoretic properties of the Ozsváth-Szabó invariants and (2) to exploit the power of the invariants to answer topological questions, particularly questions related to smooth knot concordance.
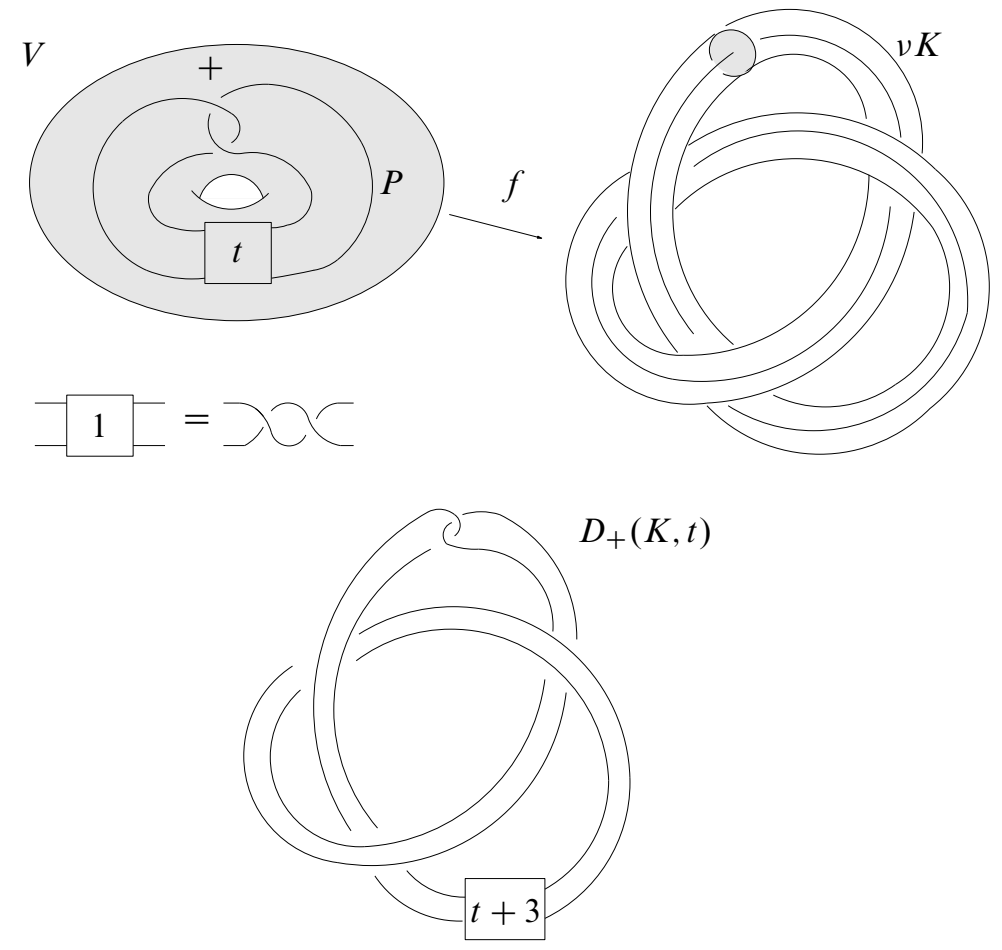

Figure 1: The positive $t$-twisted Whitehead double, $D_{+}(K, t)$, of the lefthanded trefoil. Start with a twist knot, $P$, with $t$ full twists embedded in a solid torus, $V$. The "+" indicates the parity of the clasp of $P . f$ identifies $V$ with the neighborhood of $K, v K$, in such a way that the longitude for $V$ is identified with the Seifert framing of $K$. The image of $P$ under this identification is $D_{+}(K, t)$. The 3 extra full twists in the projection of $D_{+}(K, t)$ shown arise from the writhe of the trefoil, -3 .

Suppose we have a knot $P$ embedded in a solid torus, $V$. Letting $K$ be an arbitrary knot, we can identify a tubular neighborhood of $K$ with $V$ in such a way that the longitude of $V$ (the curve on $\partial V$ generating $\left.H_{1}(V, \mathbb{Z}) \cong \mathbb{Z}\right)$ is identified with a longitude of $K$ coming from a Seifert surface. The image of $P$ under this identification is a knot, $S$, called a satellite of $K$. The knot $P$ is called the pattern for $S$, while $K$ is referred to as the companion. In this language, the positive $t$-twisted Whitehead double of a knot $K$-denoted $D_{+}(K, t)$ - is a satellite of $K$, where the pattern is a 
positive-clasped twist knot with $t$ twists. See Figure 1 for an illustration. Whitehead doubling in the context of Ozsváth-Szabó homology was first studied by Eftekhary in [6]. For other results regarding knot Floer homology and satellite knots, see Hedden [10; 11], Hedden-Ording [12], Ni [22] and Ording [23]. Before stating the main theorem, recall that associated to an integer homology three-sphere, $Y^{3}$, is the OzsváthSzabó chain complex, denoted $\widehat{C F}\left(Y^{3}\right)$ (see [29] for definitions and generalizations). Ozsváth and Szabó showed that the homology of this chain complex is an invariant of the smooth three-manifold. In [27;33], it was shown that a knot $K \subset Y^{3}$ induces a filtration of $\widehat{C F}\left(Y^{3}\right)$, and that the filtered chain homotopy type of the resulting filtered chain complex is an invariant of the knot $(Y, K)$. In the case where $Y^{3}=S^{3}$, the three-dimensional sphere, $K$ is a knot in the classical sense and the filtration of $\widehat{C F}\left(S^{3}\right)$ is denoted $\mathcal{F}(K)$. More explicitly, we have the following increasing sequence of subcomplexes:

$$
0=\mathcal{F}(K,-i) \subseteq \mathcal{F}(K,-i+1) \subseteq \ldots \subseteq \mathcal{F}(K, n)=\widehat{C F}\left(S^{3}\right) .
$$

We denote the quotient complexes $\frac{\mathcal{F}(K, j)}{\mathcal{F}(K, j-1)}:=\widehat{C F K}(K, j)$, and the homology of these quotients, denoted $\widehat{H F K}(K, j)$, are commonly referred to as the knot Floer homology groups of $K$. It follows from the fact that the filtered chain homotopy type of $\mathcal{F}(K)$ is an invariant of $K$ that the knot Floer homology groups are also knot invariants. The following theorem suggests that the knot Floer homology groups can be viewed as a "categorification" of the symmetrized Alexander-Conway polynomial, in the same spirit that the Khovanov homology groups [13] are a categorification of the Jones polynomial.

Theorem 1.1 (Ozsváth-Szabó [27], Rasmussen [33]) Let $K \subset S^{3}$ be a knot and $\Delta_{K}(T)$ its Alexander-Conway polynomial. Then

$$
\sum_{i} \chi(\widehat{H F K}(K, i)) \cdot T^{i}=\Delta_{K}(T)
$$

Suppose we look at a satellite $S$ of a knot, $K$, where the pattern $P$ in the construction represents $p$ times a generator of $H_{1}(V, \mathbb{Z})$. We have the following classical formula for the Alexander polynomial of $S$ (see [15]),

$$
\Delta_{S}(T)=\Delta_{P}(T) \cdot \Delta_{K}\left(T^{p}\right) .
$$

Since the twist knots used as pattern for the Whitehead double construction represent zero in the first homology of the solid torus, we see that the Alexander polynomial forgets the knot which we are doubling. Indeed, the Alexander polynomial of $D_{+}(K, t)$ is given by

$$
\Delta_{D_{+}(K, t)}(T)=-t \cdot T+(2 t+1)-t \cdot T^{-1},
$$


independent of $K$. In particular, the Alexander polynomial of the 0 -twisted Whitehead double of $K$ is trivial. It is thus an interesting question to ask how, if at all, the knot Floer homology of $D_{+}(K, t)$ remembers the knot $K$. In order to state our theorem, we remark that the knot Floer homology groups $\bigoplus \widehat{H F K}(K, i)$ themselves have the structure of a filtered chain complex, endowed with a differential induced from the differential on $\widehat{C F K}$. For a knot of Seifert genus one, $\widehat{H F K}(K, i)=0$ if $|i|>1$, and so this induced differential decomposes as a sum of three homomorphisms:

$$
\begin{aligned}
& d_{1}^{1}: \widehat{H F K}_{*}(K, 1) \longrightarrow \widehat{H F K}_{*-1}(K, 0), \\
& d_{1}^{0}: \widehat{H F K}_{*}(K, 0) \longrightarrow \widehat{H F K}_{*-1}(K,-1), \\
& d_{2}: \widehat{H F K}_{*}(K, 1) \longrightarrow \widehat{H F K}_{*-1}(K,-1) .
\end{aligned}
$$

(See Section 5 and Section 6 for more details.) In the following formulas and throughout the paper, we let $\mathbb{E}$ denote the field with two elements ie we use $\mathbb{Z} / 2 \mathbb{Z}$ coefficients. The term $\tau(K)$ is the Ozsváth-Szabó concordance invariant (Ozsváth-Szabó [26] and Rasmussen [33]) discussed below.

Theorem 1.2 Let $K \subset S^{3}$ be a knot with Seifert genus $g(K)=g$. Then for $t \geq 2 \tau(K)$ we have:

$$
\widehat{H F K}{ }_{*}\left(D_{+}(K, t), i\right) \cong \begin{cases}\mathbb{F}_{(1)}^{t-2 g-2} \bigoplus_{j=-g}^{g}\left[H_{*-1}(\mathcal{F}(K, j))\right]^{2} & i=1 \\ \mathbb{F}_{(0)}^{2 t-4 g-3} \bigoplus_{j=-g}^{g}\left[H_{*}(\mathcal{F}(K, j))\right]^{4} & i=0 \\ \mathbb{F}_{(-1)}^{t-2 g-2} \bigoplus_{j=-g}^{g}\left[H_{*+1}(\mathcal{F}(K, j))\right]^{2} & i=-1 .\end{cases}
$$

Whereas for $t<2 \tau(K)$ the following holds:

$$
\begin{aligned}
& \widehat{H F K}_{*}\left(D_{+}(K, t), i\right) \cong \\
& \begin{cases}\mathbb{F}_{(1)}^{2 \tau(K)-2 g-2} \oplus \mathbb{F}_{(0)}^{2 \tau(K)-t} \bigoplus_{j=-g}^{g}\left[H_{*-1}(\mathcal{F}(K, j))\right]^{2} & i=1 \\
\mathbb{F}_{(0)}^{4 \tau(K)-4 g-4} \oplus \mathbb{F}_{(-1)-2 t-1}^{4 \tau(K)} \bigoplus_{j=-g}^{g}\left[H_{*}(\mathcal{F}(K, j))\right]^{4} & i=0 \\
\mathbb{F}_{(-1)}^{2 \tau(K)-2 g-2} \oplus \mathbb{F}_{(-2)}^{2 \tau(K)-t} \bigoplus_{j=-g}^{g}\left[H_{*+1}(\mathcal{F}(K, j))\right]^{2} & i=-1 .\end{cases}
\end{aligned}
$$

Furthermore, $d_{2}=0$, regardless of $t$, and this together with the formulas above determine the filtered chain homotopy type of $\mathcal{F}\left(D_{+}(K, t)\right)$.

Remark 1.3 The precise way that $d_{2}=0$ and our formula determine $\mathcal{F}\left(D_{+}(K, t)\right)$ is discussed in Section 6. Since $\tau(K) \leq g(K)$, the reader may question what is meant by a term such as $\mathbb{F}_{(1)}^{2 \tau(K)-2 g-2}$ with negative exponent. By $\mathbb{F}_{(1)}^{-n}$, for instance, we mean the quotient of the remaining group by a subgroup of dimension $n$, supported in homological grading 1 . 
Letting $\bar{K}$ denote the reflection of a knot $K$ (ie in a given projection for $K, \bar{K}$ is obtained from $K$ by changing each over-crossing to an under-crossing), we have the following formula for the knot Floer homology (Ozsváth-Szabó [27, Proposition 3.7]),

$$
\widehat{H F K}_{*}(\bar{K}, i) \cong \widehat{H F K}_{-*}(K,-i) \text {. }
$$

In light of the equality,

$$
\overline{D_{+}(\bar{K},-t)}=D_{-}(K, t)
$$

we see that Theorem 1.2 yields the complete answer for the negative-clasped doubles as well.

One should compare Theorem 1.2 with the results of [12] and [6]. [12, Proposition 2.6] computes the Floer homology of a specific Whitehead double of the $(2, n)$ torus knot while [6] equates a particular knot Floer homology group of the 0-twisted Whitehead double with another invariant, the longitude Floer homology. Theorem 1.2 is a significant improvement over either of these results and over any other results concerning the Floer homology of satellite knots. In fact, the above theorem is a complete answer to the question of the knot Floer homology of Whitehead doubles: it handles all values of the twisting parameter, $t$, and all the Floer homology groups. Moreover, we are able to use our formula iteratively.

\subsection{Concordance invariants}

Whitehead doubles have played an interesting role in the study of knot concordance, where they highlight the distinction between the smooth and topological four-ball genus. Moreover, several fundamental open questions in the field of four-dimensional topological surgery are equivalent to questions related to Whitehead doubling (see Freedman-Quinn [8] and Freedman [7]).

Before going further, recall that the smooth four-ball genus, denoted $g_{4}(K)$, of a knot $K$ is the minimum genus of any smoothly properly embedded surface, $(F, \partial F)$, in the four-dimensional ball whose restriction to $\partial F$ is the given knot in $S^{3}$. A knot is said to be smoothly slice if its smooth four-ball genus is zero. Two knots, $K_{1}, K_{2}$ are said to be smoothly concordant if $K_{1} \#-\bar{K}_{2}$ is smoothly slice. Here $-K$ denotes the knot $K$ with reversed orientation. It can be shown that concordance is an equivalence relation on the set of knots and that under this equivalence the set of knots has the structure of an abelian group, the group operation being connected sum, $K_{1} \# K_{2}$. We denote this group, the smooth concordance group of knots, by $\mathcal{C}$. We can repeat all the above definitions in the topological category, where we require surfaces to be topologically locally flatly embedded. In this case we denote the (topological) four-ball genus and concordance group by $g_{4}^{\text {top }}(K)$ and $\mathcal{C}_{\text {top }}$, respectively. 
Whitehead doubling is an easy way to produce non-trivial topologically slice knots, as indicated by the following theorem.

Theorem 1.4 (Freedman [8]) Let $K \subset S^{3}$ be knot which satisfies $\Delta_{K}(T)=1$. Then $K$ is topologically slice. That is, $g_{4}^{\text {top }}(K)=0$.

As mentioned, the 0 -twisted Whitehead double of any knot satisfies $\Delta_{D_{ \pm}(K, 0)}(T)=1$, and hence these knots are topologically slice. While it is easy to see that the Whitehead double of a smoothly slice knot is also smoothly slice, it was shown by several authors that many Whitehead doubles are not smoothly slice (Akbulut-Matveyev [1], GompfStipsicz [4] and Rudolph [35]). The existence of a topologically slice knot which is not smoothly slice is interesting in its own right, as it implies the existence of an exotic smooth structure on $\mathbb{R}^{4}$ (see [9] for a proof). It is an open question (see Kirby's list [14, Problem 1.38]) whether the Whitehead double of $K$ is smoothly slice only when $K$ is smoothly slice.

From the knot Floer homology filtration, we can produce an integer-valued knot invariant $\tau(K)$ useful for the study of smooth knot concordance. To define it, recall that the Floer homology of the three-sphere is isomorphic to $\mathbb{F}$, supported in homological grading zero. Thus, one can define the following:

$$
\tau(K)=\min \left\{j \in \mathbb{Z} \mid i_{*}: H_{*}(\mathcal{F}(K, j)) \longrightarrow H_{*}\left(\widehat{C F}\left(S^{3}\right)\right) \text { is non-trivial }\right\} .
$$

Ozsváth and Szabó [26] and Rasmussen [33] showed that $\tau(K)$ is an invariant of the smooth concordance class of $K$, and that it provides a bound for the smooth 4-genus of $K$ :

$$
|\tau(K)| \leq g_{4}(K) .
$$

Moreover, $\tau(K)$ is additive under connected sum of knots, and hence provides a homomorphism $\mathcal{C} \rightarrow \mathbb{Z}$. It is important to note that the knot Floer homology groups of $K$ are in general not sufficient to determine $\tau(K)$ since its definition relies on a more detailed knowledge of the knot filtration $\mathcal{F}(K)$.

Theorem 1.2 indicates that the dependence of the Floer homology of $D_{+}(K, t)$ on the twisting parameter is determined by $\tau(K)$. In fact, a key ingredient used to determine the filtered chain homotopy type of $\mathcal{F}\left(D_{+}(K, t)\right)$ is the following Theorem.

\section{Theorem 1.5}

$$
\tau\left(D_{+}(K, t)\right)= \begin{cases}0 & \text { for } t \geq 2 \tau(K) \\ 1 & \text { for } t<2 \tau(K)\end{cases}
$$


As a corollary, we can determine the iterated 0 -twisted Whitehead doubles which $\tau$ can be used to show are not smoothly slice. We let $D_{+}^{i}(K)$ denote the $i$ th iterated $0-$ twisted Whitehead double of $K$ ie $D_{+}^{1}(K)=D_{+}(K, 0)$ and $D_{+}^{i}(K)=D_{+}\left(D_{+}^{i-1}(K), 0\right)$.

Corollary 1.6 $\tau\left(D_{+}^{i}(K)\right) \neq 0$ if, and only if, $\tau(K)>0$. Hence, if $\tau(K)>0$ then $D_{+}^{i}(K)$ is not smoothly slice for every $i$.

The above theorem and corollary should be compared with results of Livingston-Naik [19] which determine $\tau\left(D_{+}(K, t)\right)$ for all $t$ outside a finite interval. Using the Floer homology of the branched double cover of $D_{+}(K, 0)$, Manolescu-Owens [20] are able to show that $D_{+}(K, 0)$ is not slice if $\tau(K)>0$. However, they were unable to determine whether iterated doubles were slice since $\tau$ of these knots was unknown except in the cases computed by Livingston and Naik.

We should also remark that in the case where the companion knot is the $(2, n)$ torus knot, the above result follows from Hedden-Ording [12]. Indeed the main purpose of [12] was to show that $\tau(K)$ does not equal half the Rasmussen concordance invariant, $s(K)$ [34]. We believe that Whitehead doubles of knots with $\tau(K) \neq 0$ will provide further examples of knots with $2 \tau(K) \neq s(K)$.

In a related direction, the results of [19] and [12] indicate that there are two invariants associated to a knot:

$$
\begin{aligned}
& t_{s}(K)=\min \left\{t \in \mathbb{Z} \mid s\left(D_{+}(K, t)=0\right\} .\right. \\
& t_{\tau}(K)=\min \left\{t \in \mathbb{Z} \mid \tau\left(D_{+}(K, t)=0\right\} .\right.
\end{aligned}
$$

It follows from the fact that $s$ and $\tau$ are smooth concordance invariants that $t_{s}, t_{\tau}$ are also invariants of the smooth concordance class of $K$. However, Theorem 1.5 shows that $t_{\tau}(K)=2 \tau(K)$, and hence provides no new information. Preliminary calculations indicate that this is not the case with $t_{s}$ and we consider the question of the behavior of $t_{s}$ interesting.

\subsection{Applications and Examples: Gluing knot complements}

In the final section of the paper we use our formula for a few simple applications. For both its own interest, and to illuminate our theorem, we first present a closed formula for the Floer homology of the iterated 0 -twisted doubles of the figure-eight knot.

Perhaps more interesting, we use our formula in conjunction with a theorem of Ozsváth and Szabó to determine the Floer homology, $\widehat{H F}\left(S_{+1}^{3}\left(D_{+}(K, t)\right)\right.$, where $S_{+1}^{3}\left(D_{+}(K, t)\right)$ is the three-manifold obtained by +1 Dehn surgery on $D_{+}(K, t)$. 
These manifolds are of particular interest, since some can also be obtained by a gluing of knot complements. Indeed, if we let $T$ denote the right-handed trefoil, then +1 surgery on $D_{+}(K,-1)$ is the same manifold as that obtained from $S^{3}-K$ and $S^{3}-T$ by identifying their boundary tori via the identification:

$$
\mu_{K} \leftrightarrow \lambda_{T} \quad \lambda_{K} \leftrightarrow \mu_{T}
$$

where $\mu_{K}, \lambda_{K}$ (resp. $\mu_{T}, \lambda_{T}$ ) are the meridian and longitude for $K$ (resp. $T$ ). Our result provides the first closed formula for the Ozsváth-Szabó Floer homology of any family of manifolds obtained from this type of gluing operation (though see Eftekhary [5] for some partial results).

Organization The next section of the paper is devoted to finding an efficient Heegaard diagram for Whitehead doubles. In Section 3 we analyze this diagram and prove that a particular Floer homology group of the Whitehead double is isomorphic to the Floer homology of the meridian of $K$, viewed as a knot in the manifold obtained by $t$-surgery on $K$. Section 4 computes these groups for sufficiently large values of the twisting parameter, determining $\widehat{H F K}\left(D_{+}(K, t), 1\right)$ for large $|t|$. We then use the skein exact sequence for knot Floer homology to calculate $\widehat{H F K}\left(D_{+}(K, t), 1\right)$ for the remaining $t$. In the course of applying the skein sequence, we will determine $\tau\left(D_{+}(K, t)\right)$. Section 6 studies the remaining Floer homology group, and the "higher differentials" involved in determining the filtered chain homotopy type of $\mathcal{F}\left(D_{+}(K, t)\right)$, thus proving Theorem 1.2. The final section of the paper is dedicated to examples and applications of the main theorem.

Acknowledgments I wish to thank Eaman Eftekhary, Philip Ording, Peter Ozsváth, Jacob Rasmussen, and Zoltan Szabó for interesting conversations. I am also deeply indebted to the referee for a careful reading and many useful suggestions and comments.

\section{A Heegaard diagram for Whitehead doubles}

In this section we recall the definition of a compatible Heegaard and introduce an efficient Heegaard diagram for the Whitehead doubles. We do not review the basics of knot Floer homology (in particular we assume familiarity with the boundary operator, the definition of the knot filtration, etc). For an introduction to Heegaard diagrams for knots and computing knot Floer homology from Heegaard diagrams, see Hedden [11, Chapter 2]. 
Definition 2.1 A compatible doubly-pointed Heegaard diagram for a knot $\left(Y^{3}, K\right)$ (or simply a Heegaard diagram for $\left(Y^{3}, K\right)$ ) is a collection of data

$$
\left(\Sigma,\left\{\alpha_{1}, \ldots, \alpha_{g}\right\},\left\{\beta_{1}, \ldots, \beta_{g}\right\}, w, z\right),
$$

where

- $\Sigma$ is an oriented surface of genus $g$, the Heegaard surface,

- $\left\{\alpha_{1}, \ldots, \alpha_{g}\right\}$ are pairwise disjoint, linearly independent embedded circles (the $\alpha$ attaching circles) which specify a handlebody, $U_{\alpha}$, bounded by $\Sigma$,

- $\left\{\beta_{1}, \ldots, \beta_{g}\right\}$ are pairwise disjoint, linearly independent embedded circles which specify a handlebody, $U_{\beta}$, bounded by $\Sigma$ such that $U_{\alpha} \cup_{\Sigma} U_{\beta}$ is diffeomorphic to $Y^{3}$,

- $K$ is isotopic to the union of two arcs joined along their common endpoints $w$ and $z$. These arcs, $t_{\alpha}$ and $t_{\beta}$, are properly embedded and parallel to $\Sigma$ in the $\alpha$ and $\beta$-handlebodies, respectively.

Remark 2.2 This definition is slightly different than what was originally given in Ozsváth-Szabó [27]. For a leisurely discussion of Heegaard diagrams, the two definitions and their equivalence, see Hedden [11]. Note, too, that we are thinking of knots which may not be embedded in the three-sphere, $S^{3}$. If we refer to a knot in $S^{3}$ we will drop $Y^{3}$ from the notation.

\subsection{A diagram for Whitehead doubles}

Knot Floer homology is defined in terms of the doubly-pointed Heegaard diagram described above. Thus, in order to study Whitehead doubles, we first find a compatible diagram.

We begin by outlining our strategy for producing a diagram for Whitehead doubles (or more general satellites). This construction will be similar to that used by Eftekhary in [6]. We begin with two Heegaard diagrams, one corresponding to the pattern knot and one to the companion, see Figure 2 and Figure 4. On the genus 2 diagram for the pattern, we have $3 \alpha$ attaching curves, chosen so that the diagram with only $\alpha_{1}$ present specifies a solid torus, $V$. The remaining two $\alpha$ curves (labeled $\mu_{P}$ and $\lambda_{P}$ in Figure 2) specify a meridian/longitude framing of the boundary of $V$. On the genus $g$ diagram for the companion knot, $K$, we have $g+1 \alpha$ attaching curves. These $\alpha$ curves are chosen so that the diagram with $\alpha_{1}^{\prime}, \ldots, \alpha_{g-1}^{\prime}$ specifies $S^{3}-K$. The remaining two $\alpha$ curves (labeled $\mu_{K}$ and $\lambda_{K}$ in Figure 4) specify a meridian/longitude framing for $K$. As the pairs $\left(\mu_{P}, \lambda_{P}\right)$ and $\left(\mu_{K}, \lambda_{K}\right)$ are framings for the boundary 
tori of $V$ and $S^{3}-K$, respectively, they necessarily intersect in a single point, ie $\mu_{P} \cap \lambda_{P}=\{1$ point $\}, \mu_{K} \cap \lambda_{K}=\{1$ point $\}$. We form the connected sum of the two Heegaard diagrams near these points, in the sense that we take the connected sum of both the surfaces and the framing curves, see Figure 5. The connected sum of diagrams corresponds to identifying the boundary tori of the two three-manifolds. The homeomorphism identifying the tori depends both on the $\alpha$ curves used in the framing, and how they are connected. For the calculations of knot Floer homology found later in the text, it will be beneficial to treat the Heegaard diagrams discussed in this section as specifying two different manifolds each. This can be done by deleting one or the other of the $\alpha$ curves parameterizing each boundary torus. The resulting diagram can then be viewed as a Heegaard diagram for a closed three-manifold. The chain complex for the Floer homology of the Whitehead double will decompose nicely along the chain complexes associated to the Heegaard diagrams obtained by deleting the various framing curves $\mu_{P}, \lambda_{P}, \mu_{K}, \lambda_{K}$. With the idea in place, we begin.

Figure 2 depicts the Heegaard diagram associated to the pattern knot, $P$. As mentioned above we can interpret the diagram as two diagrams:

$$
\begin{aligned}
\operatorname{hd}(P) & =\left(\Sigma_{2},\left\{\alpha_{1}, \alpha_{2}=\lambda_{P}\right\},\left\{\beta_{1}, \beta_{2}=\mu\right\}, w, z\right) \\
\operatorname{hd}(\operatorname{Hopf}) & =\left(\Sigma_{2},\left\{\alpha_{1}, \alpha_{2}=\mu_{P}\right\},\left\{\beta_{1}, \mu\right\}, w, z\right) .
\end{aligned}
$$

Note that hd $(P)$ specifies the pattern knot in $S^{3}$, while the diagram hd(Hopf) specifies the knot in $S^{1} \times S^{2}$ shown in Figure 3. Note also that $\lambda_{P} \cap \mu_{P}=\{1$ point $\}$, which we draw on the diagram as a black hole. (The terminology hd(Hopf) is explained as follows: In [27], Ozsváth and Szabó describe a way to associate a null-homologous knot $\left(\#|L|-1 S^{1} \times S^{2}, k(L)\right)$ to a link $\left(S^{3}, L\right)$ of $|L|$ components. Our notation is explained by the fact that the Heegaard diagram specifies the knot $\left(S^{1} \times S^{2}, k(L)\right)$ associated to the two-component Hopf link.)

We now consider a Heegaard diagram for the companion knot, $K$. See Figure 4. In addition to the requirements of Definition 2.1, for this diagram we require that one of the $\alpha$ attaching curves, $\mu_{K}$, is a meridian for $K$ (so that the diagram without $\mu_{K}$ specifies the knot complement $S^{3}-K$ ). This added requirement allows us to draw a framed longitude for $K$ embedded on the Heegaard surface as follows: connect $z^{\prime}$ to $w^{\prime}$ by a small arc, $t_{\alpha}$, which intersects only $\mu_{K}$ and an arc $t_{\beta}$ which only intersects the $\alpha$ curves. The union $\lambda_{K}=t_{\alpha} \cup_{\left\{z^{\prime}, w^{\prime}\right\}} t_{\beta}$ is a longitude for the companion knot. It is clearly embedded on the surface and hence we view it as an attaching curve for the $\alpha$-handlebody. With this extra curve, the genus $g$ diagram has too many $\alpha$ curves, since $\lambda_{K}$ and $\mu_{K}$ are really a meridian/longitude framing of the boundary of $S^{3}-K$. 


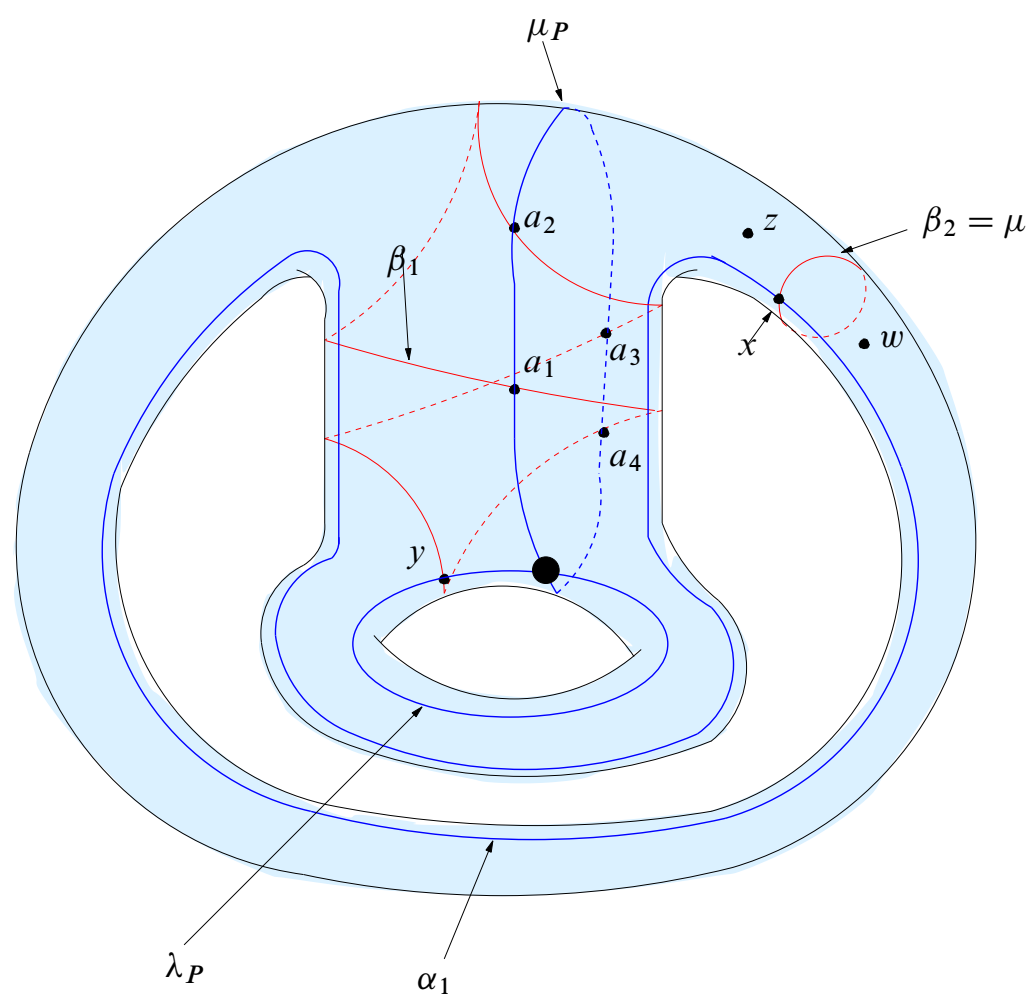

Figure 2: Genus 2 Heegaard diagram for the pattern knot in the Whitehead double construction. It can be viewed as two Heegaard diagrams, as described in the text, depending on whether we use the $\alpha$ curve $\lambda_{P}$ or $\mu_{P}$. Note the large black disc where $\lambda_{P}$ intersects $\mu_{P}$ - the Heegaard diagram for the companion knot will be connected to this diagram along the black disc.

As above, we view the diagram as two diagrams:

$$
\begin{array}{r}
\operatorname{hd}(K)=\left(\Sigma_{g},\left\{\alpha_{1}^{\prime}, \ldots, \alpha_{g}^{\prime}=\mu_{K}\right\},\left\{\beta_{1}^{\prime}, \ldots, \beta_{g}^{\prime}\right\}, w^{\prime}, z^{\prime}\right) \\
\operatorname{hd}\left(S_{t}^{3}(K), \mu_{K}\right)=\left(\Sigma_{g},\left\{\alpha_{1}^{\prime}, \ldots, \alpha_{g}^{\prime}=\lambda_{K}\right\},\left\{\beta_{1}^{\prime}, \ldots, \beta_{g}^{\prime}\right\}, w^{\prime}, z^{\prime \prime}\right) .
\end{array}
$$

hd $(K)$ is simply a diagram for the companion knot in $S^{3}$ with the last $\alpha$ curve a meridian for $K$. The second diagram no longer specifies $S^{3}$, but instead the manifold obtained by $t$-surgery on the companion knot, $S_{t}^{3}(K)$, where $t$ is the framing of the longitude $\lambda_{K}$, see Figure 3 . We can vary the framing by letting $\lambda_{K}$ circle more or fewer times around the meridian, but $\lambda_{K} \cap \mu_{K}=\{1$ point $\}$, regardless of the framing. Note, however, that in order to specify $t$ as an integer, we must decide which longitude 

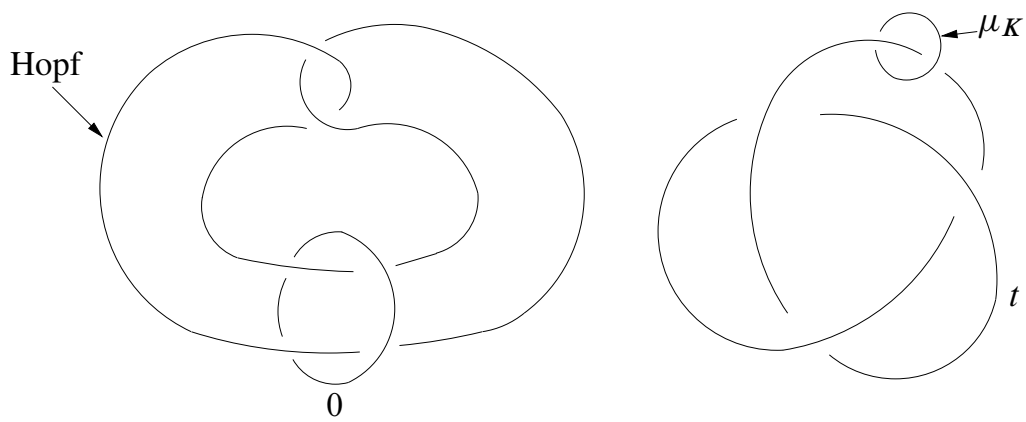

Figure 3: The "knotification" of the two-component positive Hopf link, and the knot $\left(S_{t}^{3}(K), \mu_{K}\right)$.

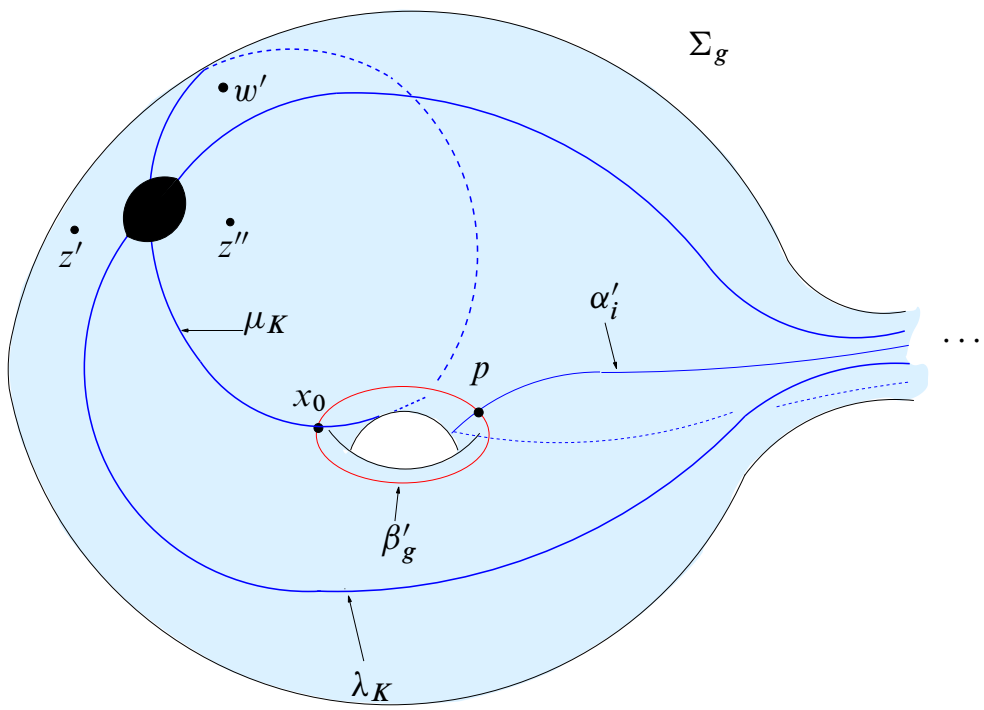

Figure 4: Heegaard diagram for an arbitrary companion knot, $K$, in the Whitehead double construction. We show only the last segment of the diagram which includes a meridian for $K$. As before, it is actually two Heegaard diagrams depending on whether we use the $\alpha$ curve $\lambda_{K}$ or $\mu_{K}$. Again we denote with a large black disc the intersection of $\lambda_{K}$ and $\mu_{K}$.

should be called the 0 -framed longitude. We adopt the standard convention that the $0-$ framing is the one specified by a Seifert surface.

The notation $\operatorname{hd}\left(S_{t}^{3}(K), \mu_{K}\right)$ is explained by the fact that the knot in $S_{t}^{3}(K)$ determined by $w^{\prime}$ and $z^{\prime \prime}$ is the meridian of $K$. Again, see Figure 3. To see this, 
simply connect $w^{\prime}$ to $z^{\prime \prime}$ by arcs in the $\alpha$ and $\beta$ handlebodies for $\operatorname{hd}\left(S_{t}^{3}(K), \mu_{K}\right)$ to recover a knot isotopic to $\mu_{K}$. It should be noted that $\left(S_{t}^{3}(K), \mu_{K}\right)$ is not a nullhomologous knot. However, when $t \neq 0,\left(S_{t}^{3}(K), \mu\right)$ is rationally null-homologous ie $[\mu]=0 \in H_{1}\left(S_{t}^{3}(K), \mathbb{Q}\right)$. In this case Ozsváth and Szabó have defined knot Floer homology groups associated to $\left(S_{t}^{3}(K), \mu_{K}\right)$, see Ozsváth-Szabó [31].

Out of the four Heegaard diagrams $\operatorname{hd}(P), \operatorname{hd}(\operatorname{Hopf}), \operatorname{hd}(K), \operatorname{hd}\left(S_{t}^{3}(K), \mu\right)$ we form a single diagram for the $t$-twisted Whitehead double $D_{+}(K, t)$ by the following construction, which can be found in Eftekhary [6].

We first describe the surface. Start by embedding the genus two surface for $P$ inside the $\alpha$ handlebody specified by $\mathrm{hd}(K)$. This is shown in Figure 5.

Next form the connected sum of the outside surface with the inside surface. We form the connected sum in a neighborhood of the intersection points $\lambda_{P} \cap \mu_{P}$ and $\lambda_{K} \cap \mu_{K}$, respectively. The resulting surface has genus $g+2$, where $g$ is the genus of the diagram for $K$.

Remark 2.3 In order to form the connected sum of surfaces in this way, we must be careful to discuss the orientations on the inner and outer surfaces. In particular, since one surface is embedded inside the other, the orientations of the two surfaces with respect to the page will be different. That is, the orientation for the outer (companion knot) Heegaard surface is such that it agrees with the standard orientation of the page in Figure 4. The orientation for the inner (pattern knot) surface is such that it is opposite the standard orientation of the page in Figure 2. This orientation convention in turn forces the convention on multiplicities for domains of Whitney disks, as shown in Figure 6.

Let us now specify the attaching curves. The $\beta$ attaching curves will be exactly the $\beta$ curves present on the original diagrams. The $\alpha$ curves will also be those present on the original diagrams, except that we connect the longitude and meridian curves, ie $\lambda_{P} \# \lambda_{K}$ and $\mu_{P} \# \mu_{K}$. We do this so that the attaching disks for $\lambda_{P} \# \lambda_{K}$ and $\mu_{P} \# \mu_{K}$ "fill in" the space between the boundary tori associated to the solid torus $V$ and to $S^{3}-K$ which is left after forming the connect sum of $\Sigma_{g}$ and $\Sigma_{2}$. Finally, for the basepoints we use the points $z$ and $w$ from the pattern. Summarizing, we have a diagram:

$$
\begin{aligned}
& \operatorname{hd}\left(D_{+}(K, t)\right)= \\
& \quad\left(\Sigma_{g+2},\left\{\alpha_{1}, \alpha_{1}^{\prime} \ldots, \alpha_{g-1}^{\prime}, \lambda_{P} \# \lambda_{K}, \mu_{P} \# \mu_{K}\right\},\left\{\beta_{1}, \mu, \beta_{1}^{\prime} \ldots, \beta_{g}^{\prime}\right\}, w, z\right) .
\end{aligned}
$$

It must be verified that the diagram is compatible with $D_{+}(K, t)$. We first observe that the three-manifold specified by the diagram is $S^{3}$. This is proved by the sequence 


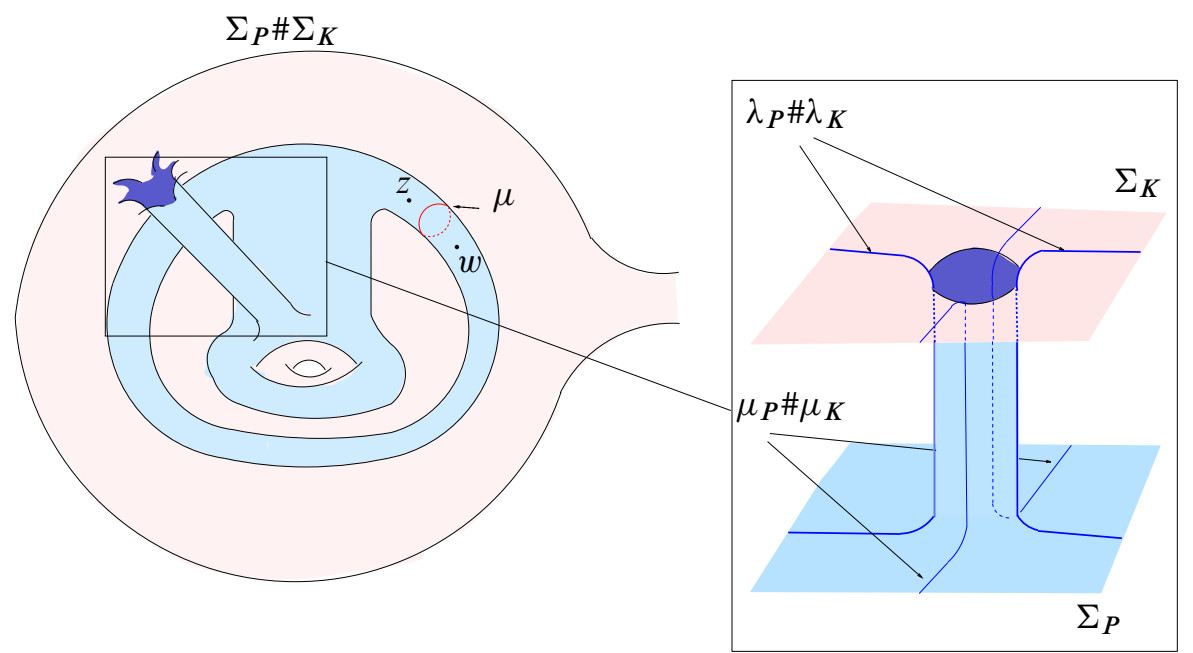

Figure 5: Depiction of the connected sum of diagrams used to obtain a Heegaard diagram for $D_{+}(K, t)$. The left side of the figure shows the connected sum of the surfaces for $P$ and $K$ along the black discs, with the diagram for $P$ (Figure 2) embedded in the $\alpha$ handlebody for $K$ (Figure 4). All curves have been omitted except the meridian for the Whitehead double, $\mu$. The right side of the figure is a closer look at the connect sum tube, and illustrates how the meridian/longitude $\alpha$-framing curves for each diagram are connected. All other $\alpha$ and $\beta$ curves are omitted, as they are inherited without modification from the diagrams for $P$ and $K$.

of Heegaard moves shown in Figure 7. Indeed, by removing the basepoint $z$, we are free to perform the sequence of isotopies, handleslides and destabilizations shown in the figure which take us from hd $\left(D_{+}(K, t)\right.$ ) (without $z$ ) to hd $(K)$ (with a single basepoint, $w$ ). Removing the $z$ basepoint is justified since here we focus solely on the three-manifold, and $z$ is relevant only for specifying the knot. Since $h d(K)$ is a diagram for a knot in $S^{3}$, by assumption, this demonstrates that the three-manifold specified by hd $\left(D_{+}(K, t)\right)$ is $S^{3}$.

It remains to see that hd $\left(D_{+}(K, t)\right)$ specifies the Whitehead double. However, this can be easily verified by drawing a longitude for the knot specified by hd $\left(D_{+}(K, t)\right)$ in the same way longitudes were drawn for the various diagrams used in the construction. Indeed, the longitude $\lambda_{P}$ for the solid torus $V$ in which $P$ was embedded is now isotopic to a $t$-framed longitude for the companion $\lambda_{K}$ via an isotopy along the attaching disk for $\lambda_{K} \# \lambda_{P}$. It follows that the knot is isotopic to the $t$-twisted Whitehead double of $K$. This completes the construction of the Heegaard diagram for $D_{+}(K, t)$. 
Companion

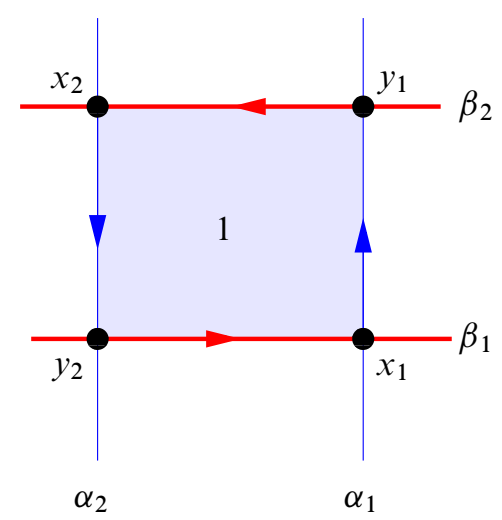

Pattern

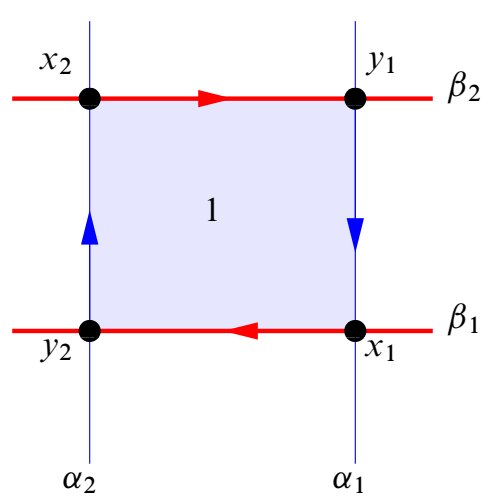

Figure 6: Orientation conventions for domains of Whitney disks. Shown is the domain of a Whitney disk with multiplicity one. If this domain were present on the subsurface of the Heegaard diagram coming from the companion knot, the induced orientation on the boundary of the domain would be the standard counterclockwise orientation. This in turn would force the corresponding Whitney disk to connect $\mathbf{x}=\left\{x_{1}, x_{2}\right\}$ to $\mathbf{y}=\left\{y_{1}, y_{2}\right\}$. If, on the other hand, the domain were present on the pattern knot subsurface, its boundary would have the clockwise orientation. This, in turn, would force the disk to connect $\mathbf{y}=\left\{y_{1}, y_{2}\right\}$ to $\mathbf{x}=\left\{x_{1}, x_{2}\right\}$.

Remark 2.4 Lipshitz's [17] thesis develops a Heegaard Floer invariant for threemanifolds with parameterized boundary. We can understand the diagrams presented here from his perspective as follows. By removing a disc from each Heegaard surface in a neighborhood of the intersection point between the final two $\alpha$ curves (the black hole in Figure 2 and Figure 4) we obtain a Heegaard surface with boundary. The final two $\alpha$ curves parameterizing our boundary then become properly embedded essential arcs on the punctured Heegaard surface. This is the diagram used by Lipshitz to define his invariant.

\section{Identification of $\widehat{H F K}\left(D_{+}(K, t), 1\right)$ with $\widehat{H F K}\left(S_{t}^{3}(K), \mu_{K}\right)$}

In this section we examine the Heegaard diagram for the Whitehead doubles constructed in the previous section. The discussion begins by examining the generators of the knot Floer homology chain complex and separating them into their respective filtration 
A

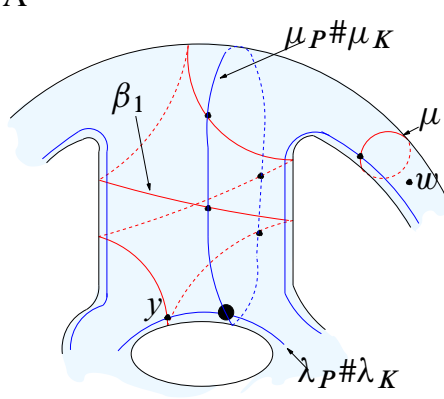

$\mathrm{C}$

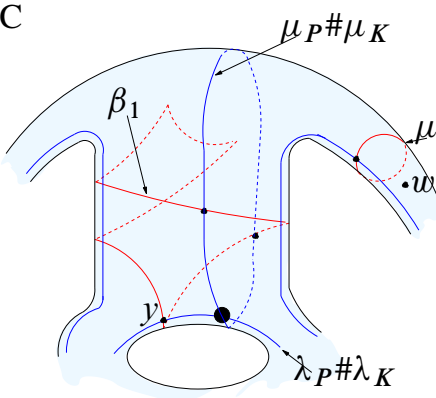

$\mathrm{E}$

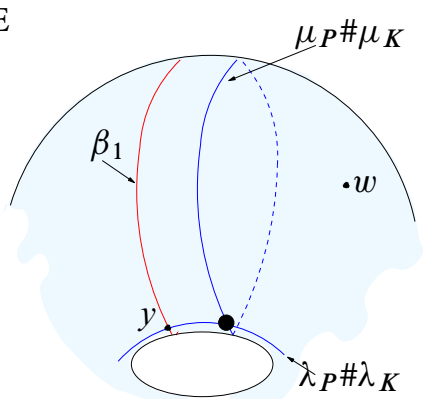

B

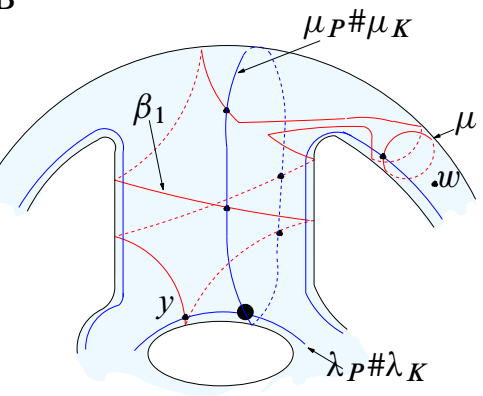

$\mathrm{D}$

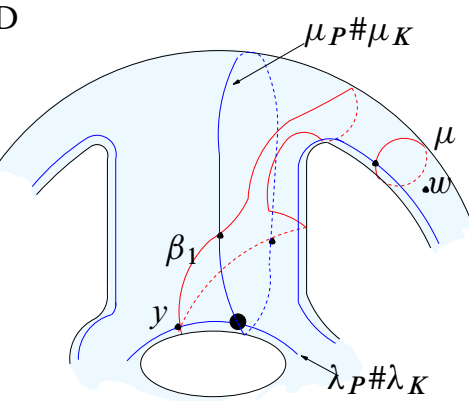

F

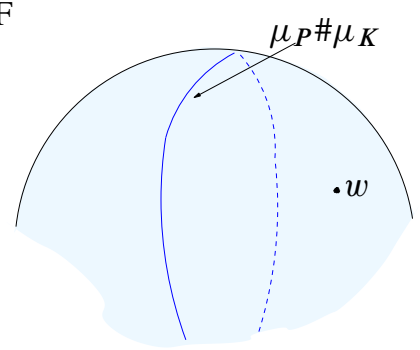

Figure 7: Heegaard moves demonstrating that hd $\left(D_{+}(K, t)\right)$ specifies $S^{3}$ (note that we have omitted the basepoint, $z$, as discussed in the text). (A) Begin with hd $\left(D_{+}(K, t)\right)$. (B) Handleslide $\beta_{1}$ over $\mu$. (C) Perform an isotopy. (D) Another handleslide of $\beta_{1}$ over $\mu$ and isotopy of $\beta_{1}$. (E) Destabilization (handle-cancellation) of $\mu$ and $\alpha_{1}$. (F) Destabilization of $\beta_{1}$ with $\lambda_{P} \# \lambda_{K}$. All Heegaard moves occur in the complement of the basepoint $w$, useful for determining gradings in Section 4.

levels. Focusing attention on the top filtration level, there will be a natural identification 
of the chain complexes:

$$
\widehat{C F K}\left(D_{+}(K, t), 1\right)=\underset{\left\{\mathfrak{s}_{i} \in \operatorname{Spin}^{c}\left(S_{t}^{3}(K)\right\}\right.}{ } \widehat{C F K}\left(S_{t}^{3}(K), \mu_{K}, \mathfrak{s}_{i}\right),
$$

where the second chain complex is the direct sum of the knot Floer homology chain complexes associated to the meridian of the companion knot, $\mu_{K}$, viewed as a knot in the manifold obtained by $t$-framed Dehn surgery on $K, S_{t}^{3}(K)$. For our purposes, it will be sufficient to examine the cases $t \neq 0$, since the Floer homology of the 0 -twisted Whitehead double will be obtained from the skein exact sequence in Section 5. Note that the direct sum above is taken over $\operatorname{Spin}^{c}$ structures on $S_{t}^{3}(K)$ and over filtration levels induced by relative $\operatorname{Spin}^{c}$ structures on $S_{t}^{3}(K)-\mu_{K}$. Let us begin.

Our first observation is that the generators of the chain complex naturally split into two types, where the splitting is in terms of the four diagrams used to construct $\operatorname{hd}\left(D_{+}(K, t)\right)$. The types are of the form:

(1) $\{x, y\} \times \mathbf{p} \in \widehat{C F K}(P) \times \widehat{C F K}(K)$

(2) $\left\{x, a_{i}\right\} \times \mathbf{q} \in \widehat{C F K}\left(S^{1} \times S^{2}, \mathrm{Hopf}\right) \times \widehat{C F}\left(S_{t}^{3}(K)\right), i=1,2,3,4$.

The splitting occurs because the generators correspond to $(g+2)$-tuples of intersection points between the $\alpha$ and $\beta$ attaching curves, with each $\alpha$ and $\beta$ curve used exactly once. Since $\lambda_{P} \# \lambda_{K}$ and $\mu_{P} \# \mu_{K}$ are the only two attaching curves which pass through the connect sum region, the splitting is determined by the surface, $\Sigma_{2}$ or $\Sigma_{g}$, where the $\lambda_{P} \# \lambda_{K}$ component of the $(g+2)$-tuple lies.

Note Since the intersection point $\{x\}$ occurs as part of the 2-tuple corresponding to any generator of $\widehat{C F K}(P)$ or $\widehat{C F K}\left(S^{1} \times S^{2}\right.$, Hopf $)$, it will subsequently be dropped from the notation eg $\{y\}:=\{x, y\}$.

We turn our attention to understanding the relative filtration difference between pairs of generators. To do this, we identify Whitney disks connecting pairs of generators in the $(g+2)$-fold symmetric product of the Heegaard surface (recall the Heegaard surface has genus $g+2$, where $g$ is the genus of the diagram for the companion knot). In fact, it will be more convenient to identify the domains of Whitney disks, by which we mean 2-chains lying in $\Sigma_{g+2}$ with boundary in the attaching curves, and corner points contained in the $(g+2)$-tuple of intersection points representing the generators. For the equivalence of these methods, see Hedden [11], Lipshitz [16] and Rasmussen [33]. Before beginning, recall the following definition, found in Ozsváth-Szabó [29]. 
Definition 3.1 A periodic domain is a 2-chain, $\mathcal{P} \subset \Sigma$, such that the boundary of $\mathcal{P}$ consists of a linear combination of attaching circles and so that the local multiplicity at $w$ is zero i.e $\partial \mathcal{P}=\Sigma_{i=1}^{g}\left[n_{i} \alpha_{i}+m_{i} \beta_{i}\right], n_{i}, m_{i} \in \mathbb{Z}$ and $n_{w}(\mathcal{P})=0$.

If $H_{1}(Y, \mathbb{Q})=0$, it follows that there will not exist any periodic domains. Indeed, since $\operatorname{hd}\left(D_{+}(K, t)\right)$ is a diagram for $S^{3}$ it will not contain any periodic domains. However, the diagram hd(Hopf) which went into the construction of hd $\left(D_{+}(K, t)\right)$ represented $S^{1} \times S^{2}$, and this diagram contains periodic domains. A generator for the space of periodic domains on hd(Hopf) is shown in Figure 8. We will subsequently refer to this generator as $\mathcal{P}$.
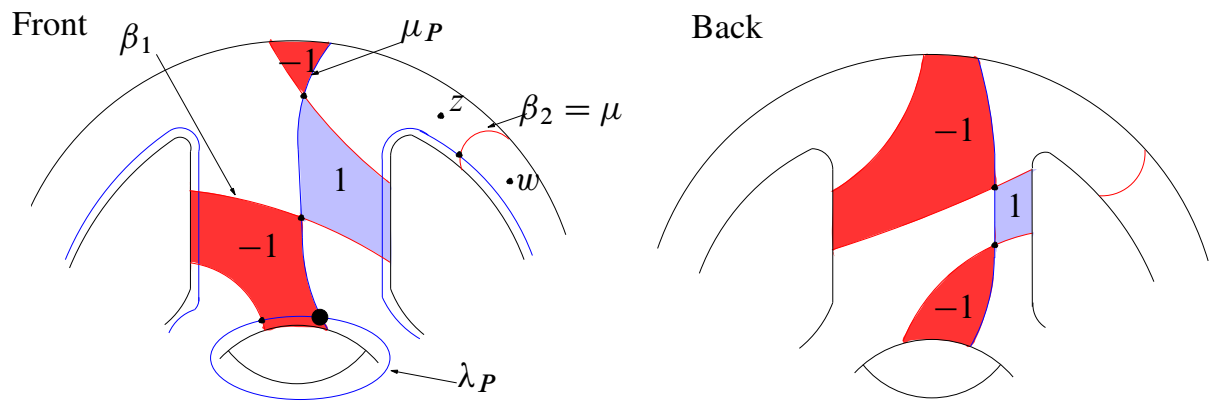

Figure 8: Illustration of the generator for the space of periodic domains for the diagram hd(Hopf). Non-zero multiplicities of the two-chain are indicated with shading. Dark (red) shading indicates multiplicity -1 , while light (blue) shading indicates multiplicity 1 .

To begin, we determine the filtration difference between pairs of points of Type (1).

Lemma 3.2 Suppose $\{y\} \times \mathbf{p}_{i},\{y\} \times \mathbf{p}_{k}, \in \widehat{C F K}(P) \times \widehat{C F K}(K)$. Then

$$
\mathcal{F}\left(\{y\} \times \mathbf{p}_{i}\right)-\mathcal{F}\left(\{y\} \times \mathbf{p}_{k}\right)=0 .
$$

Proof We first note that since $\mathbf{p}_{i}$ and $\mathbf{p}_{k}$ can be viewed as generators of $\widehat{C F K}(K)$, they can be connected by a Whitney disk, $\phi$, with domain contained in hd $(K)$. However, if $\mathbf{p}_{i}$ and $\mathbf{p}_{k}$ (viewed as generators of $\widehat{C F K}(K)$ ) lie in different filtration levels, then the boundary of $\phi$ must contain the meridian $\mu_{K}$ with non-zero multiplicity (this follows from the definition of the filtration). We can complete the domain of such a Whitney disk for $\mathbf{p}_{i}, \mathbf{p}_{k} \in \widehat{C F K}(K)$ to the domain of a disk connecting $\{y\} \times \mathbf{p}_{i}$ to $\{y\} \times \mathbf{p}_{k}$ in $\operatorname{hd}\left(D_{+}(K, t)\right)$ by forming the boundary sum of $\phi$ with $n \cdot \mathcal{P}$, where $n$ is the filtration difference of $\mathbf{p}_{i}$ and $\mathbf{p}_{k}$. The lemma follows because $n_{z}(\mathcal{P})=n_{w}(\mathcal{P})=0$.

Next, we handle the filtration difference of pairs of points of Type (2). 
Lemma 3.3 Suppose $\left\{a_{i}\right\} \times \mathbf{q}_{j},\left\{a_{i}\right\} \times \mathbf{q}_{k}, \in \widehat{C F K}\left(S^{1} \times S^{2}, \operatorname{Hopf}\right) \times \widehat{C F}\left(S_{t}^{3}(K)\right)$ Then

$$
\mathcal{F}\left(\left\{a_{i}\right\} \times \mathbf{q}_{j}\right)-\mathcal{F}\left(\left\{a_{i}\right\} \times \mathbf{q}_{k}\right)=0 .
$$

Proof At first sight it appears the method used in the proof of the preceding lemma is hopeless. Since $H^{2}\left(S_{t}^{3}(K), \mathbb{Z}\right) \neq 0$, arbitrary pairs of generators for $\widehat{C F}\left(S_{t}^{3}(K)\right)$ cannot be connected by a Whitney disk: $\mathbf{q}_{j}$ and $\mathbf{q}_{k}$ could represent different $\operatorname{Spin}^{c}$ structures on $S_{t}^{3}(K)$. However, the obstruction to finding a Whitney disk connecting $\mathbf{q}_{j}$ to $\mathbf{q}_{k}$ lies in $H^{2}\left(S_{t}^{3}(K), \mathbb{Z}\right) \cong H_{1}\left(S_{t}^{3}(K), \mathbb{Z}\right)$ which is generated by the meridian of $K, \mu_{K}$. Thus, if Whitney disks are allowed whose boundary contains $\mu_{K}$ (not an attaching curve for $\mathrm{hd}\left(S_{t}^{3}(K)\right)$, but present on the diagram for $\left.D_{+}(K, t)\right)$ then $\mathbf{q}_{j}$ and $\mathbf{q}_{k}$ can be connected regardless of their $\operatorname{Spin}^{c}$ structure. The lemma is proved by completing the domains of these Whitney disks with periodic domains, as in the proof of the previous lemma.

Now we examine the effect of varying the generator on the diagram, hd(Hopf).

Lemma 3.4 For all $\mathbf{p} \in \widehat{C F}\left(S_{t}^{3}(K)\right)$ we have:

$$
\begin{aligned}
& \mathcal{F}\left(\left\{a_{1}\right\} \times \mathbf{p}\right)-\mathcal{F}\left(\left\{a_{4}\right\} \times \mathbf{p}\right)=1, \\
& \mathcal{F}\left(\left\{a_{2}\right\} \times \mathbf{p}\right)-\mathcal{F}\left(\left\{a_{1}\right\} \times \mathbf{p}\right)=-1, \\
& \mathcal{F}\left(\left\{a_{3}\right\} \times \mathbf{p}\right)-\mathcal{F}\left(\left\{a_{4}\right\} \times \mathbf{p}\right)=-1, \\
& \mathcal{F}\left(\left\{a_{2}\right\} \times \mathbf{p}\right)-\mathcal{F}\left(\left\{a_{3}\right\} \times \mathbf{p}\right)=1 .
\end{aligned}
$$

Furthermore, there exists a pair $\mathbf{q} \in \widehat{C F K}(K)$ and $\mathbf{p} \in \widehat{C F}\left(S_{t}^{3}(K)\right)$ so that:

$$
\mathcal{F}(\{y\} \times \mathbf{q})-\mathcal{F}\left(\left\{a_{4}\right\} \times \mathbf{p}\right)=0 .
$$

Proof We prove the lemma by explicitly identifying the domains of Whitney disks connecting generators of the above form. The discussion will be aided by Figures 9, 10,11 and 12 .

Restricting attention to the Heegaard diagram hd(Hopf), pairs of generators $\left\{a_{i}\right\}$ and $\left\{a_{j}\right\}$ can be connected by Whitney disks whose domains are topologically annuli. These annuli can be completed to domains on $\operatorname{hd}\left(D_{+}(K, t)\right)$ by extending the annulus on hd(Hopf) by the domain on hd $\left(S_{t}^{3}(K)\right)$ which has multiplicity zero everywhere. See Figure 9 and Figure 10 for illustrations. The first four rows in the table below describe the annuli. The fourth column lists the Maslov index of the disk, which will be needed when gradings are discussed in the next section. The last two columns indicate the 

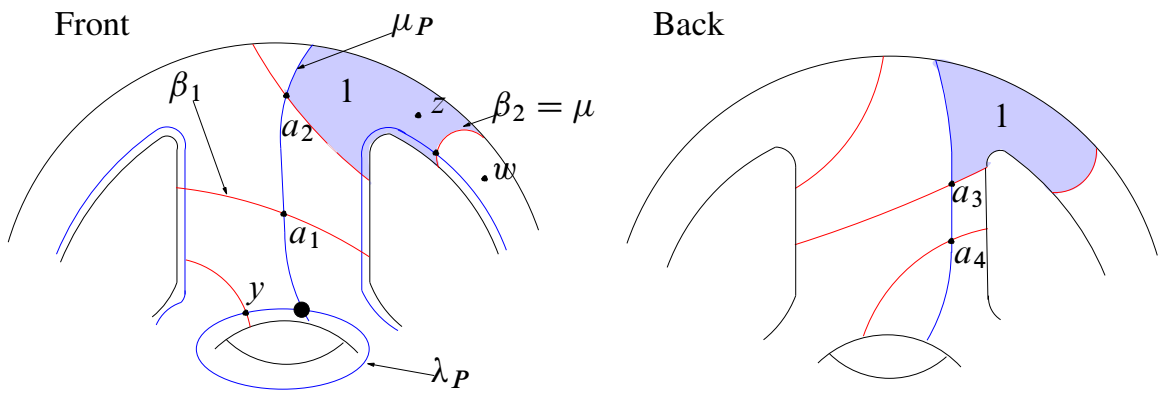

Figure 9: Illustration of the domain of a Whitney disk connecting $a_{2}$ to $a_{3}$ satisfying $n_{w}(\phi)=0, n_{z}(\phi)=1$. It is topologically an annulus, and can be shown to admit a unique holomorphic representative. This fact will be used in Section 6. Recall from Remark 2.3 that the orientation of this part of the Heegaard surface is opposite the standard orientation of the plane of the page. However, since the "back" side of the diagram displays the mirror image, the orientation of this side agrees the usual right-handed orientation of the page.

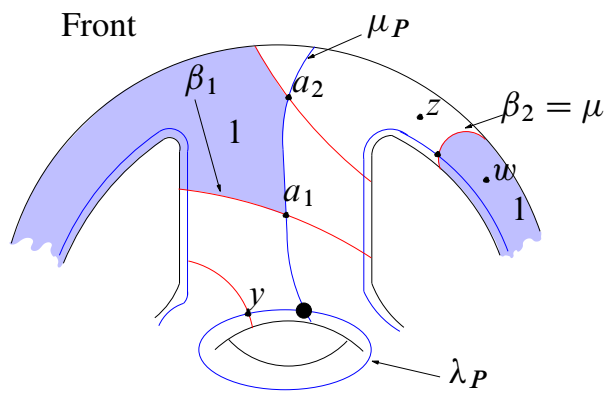

Back

Figure 10: Illustration of the domain of a Whitney disk connecting $a_{2}$ to $a_{1}$ satisfying $n_{w}(\phi)=1, n_{z}(\phi)=0$. It is topologically an annulus.

multiplicities of the domains at the points $z$ and $w$. The existence and topology of the annuli completes the first four parts of the lemma.

For the last, it is possible to connect $\{y\} \times \mathbf{q}$ to $\left\{a_{4}\right\} \times \mathbf{p}$ by a domain whose multiplicities are shown in the table, provided that $\mathbf{q}$ and $\mathbf{p}$ are chosen carefully. Indeed, the topology of the domain depends in this case on the framing, $t$. More precisely, any intersection point in $\widehat{C F K}(K)$ is of the form $\mathbf{q}=\left\{x_{0}, \mathbf{x}\right\}$, where $x_{0} \in \mu_{K} \cap \beta_{g}^{\prime}$ is the unique intersection point in $\mu_{K}$ lying on the diagram for the companion, and $\mathbf{x}$ is some $(g-1)$-tuple of intersection points on the diagram for the companion, see 


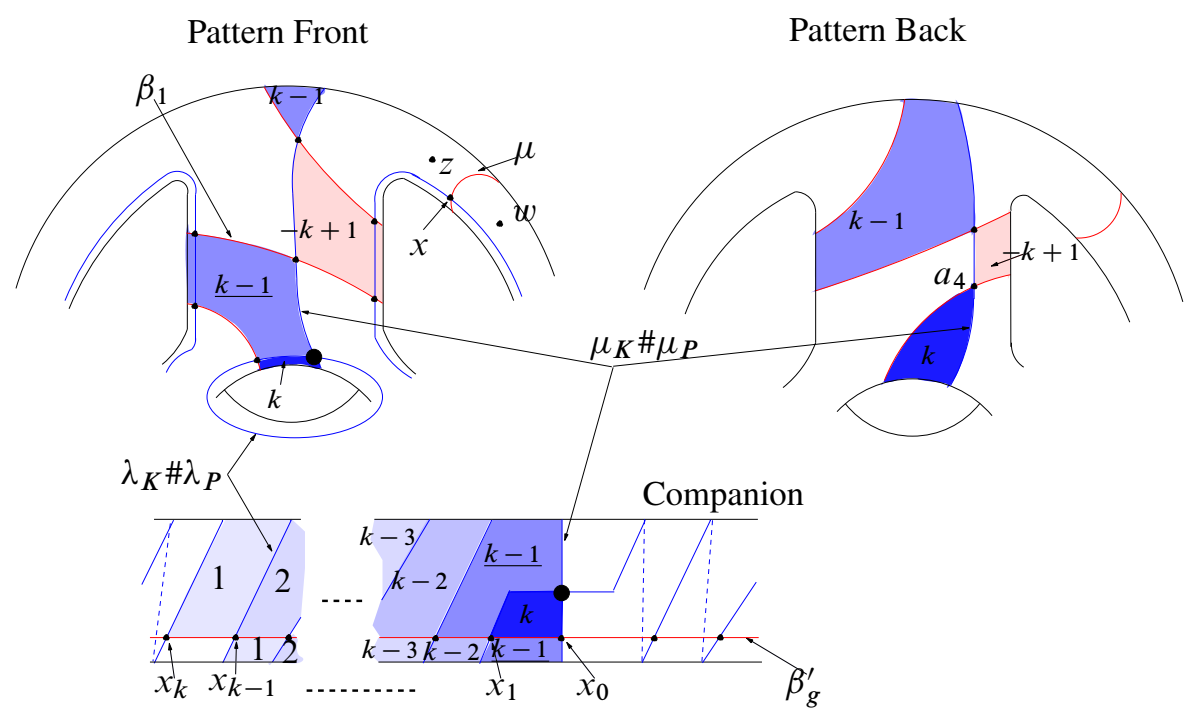

Figure 11: Illustration of domain of disk connecting $\{y\} \times\left\{x_{0}, \mathbf{x}\right\}$ to $\left\{a_{4}\right\} \times$ $\left\{x_{k}, \mathbf{x}\right\}$ for negative twisting, $t<0$. Shown are sections of the Heegaard diagram coming from the pattern and companion knots. The piece from the companion knot is the cylindrical region where the connect sum of diagrams was performed. Note that while the slope of the longitude $\lambda_{K}$ appears positive, it is part of an $\alpha$ curve and hence negative. Here $x_{0}$ is the unique intersection point in $\mu_{K} \# \mu_{P} \cap \beta_{g}^{\prime}$, and $x_{1}, \ldots, x_{k}$ are intersection points in $\lambda_{K} \# \lambda_{P} \cap \beta_{g}^{\prime}$. The small triangle with vertices $x_{0}, x_{k}$ and the connect sum disk is completed to the domain of a Whitney disk, $\phi_{y, a_{4}}$. This disk satisfies $n_{z}\left(\phi_{y, a_{4}}\right)=n_{w}\left(\phi_{y, a_{4}}\right)=0$. Note that each region which comprises the domain of $\phi_{y, a_{4}}$ is a disk with 4 corners, with the exception of the region with multiplicity marked $\mathrm{k}-1$, which is a disk with 8 corners. This fact is used in calculating that the Maslov index of $\phi_{y, a_{4}}$ is equal to 1 .

Figure 11. Furthermore, since we have assumed $t \neq 0$, there are intersection points $x_{1}, \ldots, x_{k} \in \lambda_{K} \cap \beta_{g}^{\prime}$ and small triangles connecting $x_{0}$ to $x_{k}$. Let $\mathbf{p}=\left\{x_{k}, \mathbf{x}\right\}$. Extending the domain of the triangle by zero to the rest of the diagram for the companion, we can complete the triangle to the domain of a Whitney disk, $\phi_{y, a_{4}}$ connecting $\{y\} \times \mathbf{q}$ to $\left\{a_{4}\right\} \times \mathbf{p}$. This is shown for $t<0$ in Figure 11. Note that $n_{z}\left(\phi_{y, a_{4}}\right)=n_{w}\left(\phi_{y, a_{4}}\right)=0$. For $t>0$, the same procedure produces a Whitney disk $\phi_{a_{1}, y}$ connecting $\left\{a_{1}\right\} \times \mathbf{p}$ to $\{y\} \times \mathbf{q}$ with $n_{z}\left(\phi_{a_{1}, y}\right)=1, n_{w}\left(\phi_{a_{1}, y}\right)=0$, see Figure 12 . By additivity of the filtration under concatenation of Whitney disks we have in either event that

$$
\mathcal{F}(\{y\} \times \mathbf{q})-\mathcal{F}\left(\left\{a_{4}\right\} \times \mathbf{p}\right)=0,
$$




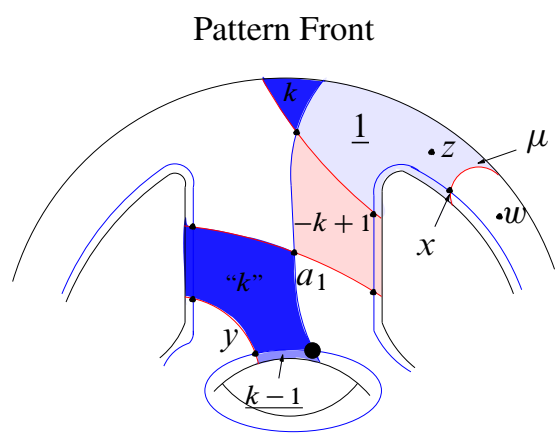

Pattern Back

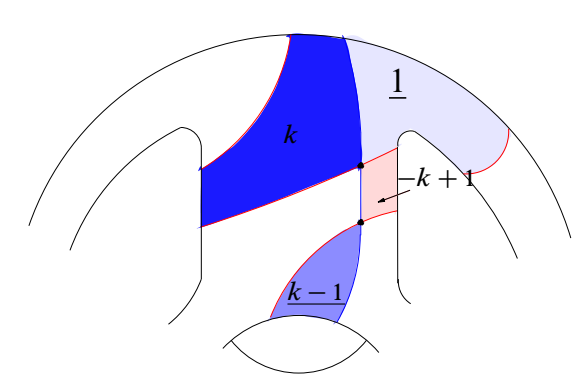

Companion

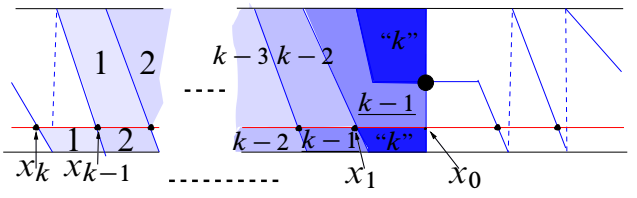

Figure 12: Illustration of the domain of a disk, $\phi_{a_{1}, y}$, connecting $\left\{a_{1}\right\} \times$ $\left\{x_{k}, \mathbf{x}\right\}$ to $\{y\} \times\left\{x_{0}, \mathbf{x}\right\}$ for $t>0$. All regions comprising the domain of $\phi_{a_{1}, y}$ are disks with 4 corners, except for the regions whose multiplicities are marked by $\underline{\mathrm{k}-1}, \underline{1}$, or " $\mathrm{k}$ ". Each of these regions is a disk with 6 corners.

as claimed.

\begin{tabular}{|c|c||c|c|c|c|}
\hline Start Pt & End Pt & $t$ & $\mu(\phi)$ & $n_{z}(\phi)$ & $n_{w}(\phi)$ \\
\hline$\left\{a_{1}\right\}$ & $\left\{a_{4}\right\}$ & all & 1 & 1 & 0 \\
\hline$\left\{a_{2}\right\}$ & $\left\{a_{1}\right\}$ & all & 1 & 0 & 1 \\
\hline$\left\{a_{3}\right\}$ & $\left\{a_{4}\right\}$ & all & 1 & 0 & 1 \\
\hline$\left\{a_{2}\right\}$ & $\left\{a_{3}\right\}$ & all & 1 & 1 & 0 \\
\hline$\{y\}$ & $\left\{a_{4}\right\}$ & $t<0$ & 1 & 0 & 0 \\
\hline$\left\{a_{1}\right\}$ & $\{y\}$ & $t>0$ & 1 & 1 & 0 \\
\hline
\end{tabular}

Thus the lemma is proved.

\subsection{Maslov Indices}

For the purposes of separating the generators of the chain complex coming from hd $\left(D_{+}(K, t)\right)$ into their respective filtration levels, the preceding lemmas provide sufficient information. However, to establish the homological gradings of the generators, we will require the Maslov indices of the disks constructed in Lemma 3.4. For this, 
it will be useful to call upon Lipshitz [16, Corollary 4.3 and Proposition 4.8], which together present a formula for the Maslov index of a Whitney disk in terms of the geometry of its domain. To state the formula we must develop some terminology. First, let us call each connected component of $\Sigma-\alpha_{1}-\cdots-\beta_{g}$ a region. Topologically, each region is a surface with boundary, and we denote regions by $\mathcal{D}$. Given a Whitney disk, $\phi$, we can express its domain, which here we will denote $\mathcal{D}_{\phi}$, as a weighted sum of the different regions $\mathcal{D}_{\phi}=\sum_{i=0}^{m} n_{i} \mathcal{D}_{i}$. In terms of this data, we can define the Euler measure of the domain of the Whitney disk, denoted $\widehat{\chi}$, by

$$
\widehat{\chi}\left(\mathcal{D}_{\phi}\right)=\sum_{i=0}^{m} n_{i}\left(\chi\left(\operatorname{int} \mathcal{D}_{i}\right)-\frac{1}{4}\left(\# \text { corner points of } \mathcal{D}_{i}\right)\right),
$$

where $\chi$ is the ordinary Euler characteristic, and where the corner points of each region $\mathcal{D}_{i}$ are counted with multiplicity. By multiplicity we mean the following: as we make a full traversal of each boundary component of $\mathcal{D}_{i}$, points are encountered where we switch from being on an $\alpha$ curve to being on a $\beta$ curve. Each time such points are encountered we add one to the corner point count, even if we encounter a given point multiple times throughout the traversal.

Given a point $p \in \alpha_{i} \cap \beta_{j}$, define the local multiplicity of $\mathcal{D}_{\phi}$ at $p$, denoted $\bar{n}_{p}\left(\mathcal{D}_{\phi}\right)$, to be the sum of the multiplicities of $\mathcal{D}_{\phi}$ in the four regions surrounding $p$. That is to say, for any intersection point $p \in \alpha_{i} \cap \beta_{j}$ there exist four (not necessarily distinct) $\mathcal{D}$ which contain $p$. If $p \in\left\{\mathcal{D}_{i} \cap \mathcal{D}_{j} \cap \mathcal{D}_{k} \cap \mathcal{D}_{l}\right\}$ then the local multiplicity $\bar{n}_{p}\left(\mathcal{D}_{\phi}\right)$ is the sum of the multiplicities, $n_{i}$, of $\mathcal{D}_{\phi}$ in these $\mathcal{D}$ :

$$
\bar{n}_{p}\left(\mathcal{D}_{\phi}\right)=n_{i}+n_{j}+n_{k}+n_{l} .
$$

The point-measure, denoted $\mu_{\mathbf{x}}$, of $\mathcal{D}_{\phi}$ at the intersection point $\mathbf{x}=\left\{x_{1}, \ldots, x_{g}\right\}$ is defined to be the weighted sum of the local multiplicities of each $x_{i} \in \mathbf{x}$ :

$$
\mu_{\mathbf{x}}\left(\mathcal{D}_{\phi}\right)=\frac{1}{4} \sum_{x_{i} \in \mathbf{x}} \bar{n}_{x_{i}}\left(\mathcal{D}_{\phi}\right)
$$

In terms of these quantities, the following formula determines the Maslov index of a Whitney disk, $\phi$, connecting $\mathbf{x}$ to $\mathbf{y}$ (Lipshitz [16, Corollary 4.3 and Proposition 4.8]):

$$
\mu(\phi)=\widehat{\chi}\left(\mathcal{D}_{\phi}\right)+\mu_{\mathbf{x}}\left(\mathcal{D}_{\phi}\right)+\mu_{\mathbf{y}}\left(\mathcal{D}_{\phi}\right) .
$$

Using this formula, we can easily establish the following Proposition.

Proposition 3.5 For the disks $\phi_{y, a_{4}}, \phi_{a_{1}, y}$ of Lemma 3.4,

$$
\mu\left(\phi_{y, a_{4}}\right)=\mu\left(\phi_{a_{1}, y}\right)=1,
$$


independent of which intersection points $\mathbf{p}=\left\{x_{k}, \mathbf{x}\right\}, \mathbf{q}=\left\{x_{0}, \mathbf{x}\right\}$ are chosen in the construction.

Proof First note that $\mathcal{D}$ which are disks with four corners contribute nothing to $\widehat{\chi}\left(\mathcal{D}_{\phi}\right)$. For $\phi_{y, a_{4}}$, the only region which is not a disk with four corners is the region whose multiplicity is marked by $\mathrm{k}-1$. This region is a disk with 8 corners, and thus

$$
\widehat{\chi}\left(\mathcal{D}_{\phi_{y, a_{4}}}\right)=(k-1) \cdot\left(1-\frac{8}{4}\right)=-k+1 .
$$

The point measure of $\phi_{y, a_{4}}$ at $\{x, y\} \times\left\{x_{0}, \mathbf{x}\right\}$ is given by

$$
\frac{1}{4}\left(\bar{n}_{y}+\bar{n}_{x_{0}}\right)=\frac{k+k-1}{4}+\frac{k+k-1}{4}=\frac{2 k-1}{2} .
$$

Note that for the point measure, we must include the point $x \in \alpha_{1} \cap \mu$ which we have been excluding from the notation. However, the local multiplicities of the domain of $\phi_{y, a_{4}}$ at $x$, like those at the points in the $(g-1)$-tuple, $\mathbf{x}$, are 0 . The point measure at $\left\{x, a_{4}\right\} \times\left\{x_{k}, \mathbf{x}\right\}$ is

$$
\frac{1}{4}\left(\bar{n}_{a_{4}}+\bar{n}_{x_{k}}\right)=\frac{k-k+1}{4}+\frac{1}{4}=\frac{1}{2} .
$$

Applying Formula (2) immediately yields $\mu\left(\phi_{y, a_{4}}\right)=1$. The case of $\phi_{a_{1}, y}$ follows similarly.

Having established the Maslov indices of all relevant Whitney disks, let us return to the filtration. Lemmas 3.2-3.4 are enough to determine the relative filtration difference between any two generators. It follows that the chain complex for $D_{+}(K, t)$ splits into three distinct filtration levels of the following form:

$$
\begin{aligned}
& \widehat{C F K}\left(D_{+}(K, t), 1\right) \sim\left\{a_{1}\right\} \times \widehat{C F}\left(S_{t}^{3}(K)\right) \widehat{C F K}\left(D_{+}(K, t), 0\right) \sim\left[\left\{a_{2}\right\} \times \widehat{C F}\left(S_{t}^{3}(K)\right)\right] \oplus \\
& {\left[\left\{a_{4}\right\} \times \widehat{C F}\left(S_{t}^{3}(K)\right)\right] \oplus[\{y\} \times \widehat{C F K}(K)] } \\
& \widehat{C F K}\left(D_{+}(K, t),-1\right) \sim\left\{a_{3}\right\} \times \widehat{C F}\left(S_{t}^{3}(K)\right),
\end{aligned}
$$

where the symbol $\sim$ means that there is a bijection between the generators of eg $\widehat{C F K}\left(D_{+}(K, t), 1\right)$ and $\left\{a_{1}\right\} \times \widehat{C F}\left(S_{t}^{3}(K)\right)$. It remains to understand the boundary operator for the chain complexes. Let us direct attention to the top filtration level for $D_{+}(K, t)$. We claim that, while a priori the chain complex $\widehat{C F K}\left(D_{+}(K, t), 1\right)$ looks 
like $\widehat{C F}\left(S_{t}^{3}(K)\right)$ as stated above, it is in fact chain homotopy equivalent to the chain complex

$$
\bigoplus_{\left\{\mathfrak{s}_{i} \in \operatorname{Spin}^{c}\left(S_{t}^{3}(K)\right\}\right.} \widehat{C F K}\left(S_{t}^{3}(K), \mu_{K}, \mathfrak{s}_{i}\right) .
$$

In other words, we have the following Theorem.

Theorem 3.6 Let $K$ be a knot in $S^{3}$. Then

$$
\widehat{H F K}\left(D_{+}(K, t), 1\right) \cong \bigoplus_{\left\{\mathfrak{s}_{i} \in \operatorname{Spin}^{c}\left(S_{t}^{3}(K)\right\}\right.} \widehat{H F K}\left(S_{t}^{3}(K), \mu_{K}, \mathfrak{s}_{i}\right),
$$

Where the latter summation is taken over $\operatorname{Spin}^{c}$ structures on $S_{t}^{3}(K) \cong \mathbb{Z} / t \mathbb{Z}$ and over filtration levels induced by $\left(S_{t}^{3}(K), \mu_{K}\right)$.

Remark 3.7 The groups $\bigoplus \widehat{H F K}\left(S_{t}^{3}(K), \mu_{K}, \mathfrak{s}_{i}\right)$ have a well-defined absolute $\mathbb{Q}$ grading which is a lift of a relative $\mathbb{Z}$-grading. The isomorphism stated above does not, in general, hold on the level of graded abelian groups. The graded statement is obtained in the next section.

Proof As previously noted, the generators of $\widehat{C F K}\left(D_{+}(K, t), 1\right)$ are of the form $\left\{a_{1}\right\} \times \widehat{C F}\left(S_{t}^{3}(K)\right)$. By definition of the knot filtration, this implies $\left\{a_{1}\right\} \times \mathbf{p}$ to $\left\{a_{1}\right\} \times \mathbf{q}$ can be connected by a Whitney disk, $\phi$, for any $\mathbf{p}, \mathbf{q} \in \widehat{C F}\left(S_{t}^{3}(K)\right)$. If we additionally require that $n_{z}(\phi)=n_{w}(\phi)=0$, then $\phi$ will be unique. Recall that the Heegaard diagram hd $\left(\left(S_{t}^{3}(K), \mu_{K}\right)\right.$ which went into the construction of hd $\left(D_{+}(K, t)\right)$ came equipped with two basepoints, $z^{\prime \prime}, w^{\prime}$. As described in Ozsváth-Szabó [29], the point $w^{\prime}$ induces a map

$$
\mathfrak{s}_{w^{\prime}}: \widehat{C F}\left(S_{t}^{3}(K)\right) \rightarrow \operatorname{Spin}^{c}\left(S_{t}^{3}(K)\right),
$$

which assigns to each generator in the chain complex a $\operatorname{Spin}^{c}$ structure. We first claim that if $\mathfrak{s}_{w^{\prime}}(\mathbf{p}) \neq \mathfrak{s}_{w^{\prime}}(\mathbf{q})$ then the Whitney disk connecting $\left\{a_{1}\right\} \times \mathbf{p}$ to $\left\{a_{1}\right\} \times \mathbf{q}$ will not admit any pseudo-holomorphic representatives. This follows from the discussion in the proof of Lemma 3.3: since $\mathfrak{s}_{w^{\prime}}(\mathbf{p}) \neq \mathfrak{s}_{w^{\prime}}(\mathbf{q})$, the domain of the Whitney disk connecting $\left\{a_{1}\right\} \times \mathbf{p}$ to $\left\{a_{1}\right\} \times \mathbf{q}$ must contain the meridian $\mu_{K}$ in its boundary a non-zero number of times. Since there are no corner points for the Whitney disk on the Heegaard diagram for the pattern (the disk has only degenerate corners at $\left\{x, a_{1}\right\}$ on hd(Hopf)), the domain of $\phi$ restricted to hd(Hopf) is simply $n \cdot \mathcal{P}, n \neq 0$ (where, as above $\mathcal{P}$ indicates the periodic domain for hd(Hopf)). Since $\mathcal{P}$ has both positive and negative multiplicities (Figure 8) the disk cannot admit any pseudo-holomorphic representatives. 
It follows that the boundary operator on $\widehat{C F K}\left(D_{+}(K, t), 1\right)$ respects the splitting along $\operatorname{Spin}^{c}$ structures that it inherits as a set from $\widehat{C F}\left(S_{t}^{3}(K)\right)$. It remains to understand the boundary operator for each $\operatorname{Spin}^{c}$ structure. Under the bijection between generators of $\widehat{C F K}\left(D_{+}(K, t), 1\right)$ and $\widehat{C F}\left(S_{t}^{3}(K)\right)$, we claim that boundary operator on $\widehat{C F K}\left(D_{+}(K, t), 1\right)$ is precisely the operator obtained from $\widehat{C F}\left(S_{t}^{3}(K)\right)$ by requiring $n_{z^{\prime \prime}}(\phi)=0$, in addition to $n_{w^{\prime}}(\phi)=0$. Since the Heegaard diagram for $S_{t}^{3}(K)$ with both basepoints $z^{\prime \prime}, w^{\prime}$ is a compatible diagram for the knot $\left(S_{t}^{3}(K), \mu_{K}\right)$, the theorem will follow from our claim and the definition of $\bigoplus \widehat{\operatorname{HFK}}\left(S_{t}^{3}(K), \mu_{K}, \mathfrak{s}_{i}\right)$. $\left\{\mathfrak{s}_{i} \in \operatorname{Spin}^{c}\left(S_{t}^{3}(K)\right\}\right.$

To prove the claim, examine the unique Whitney disk satisfying $n_{z}(\phi)=n_{w}(\phi)=0$ connecting $\left\{a_{1}\right\} \times \mathbf{p}$ to $\left\{a_{1}\right\} \times \mathbf{q}$ for any $\mathbf{p}, \mathbf{q}$ with $\mathfrak{s}_{w^{\prime}}(\mathbf{p})=\mathfrak{s}_{w^{\prime}}(\mathbf{q})$. Since the disk has no corner points on hd(Hopf), it restricts to $n \cdot \mathcal{P}$ on hd(Hopf). However, in order for $\phi$ to admit a holomorphic representative, $n=0$ since $\mathcal{P}$ has positive and negative multiplicities. Thus, the multiplicities of $\phi$, like those of $\mathcal{P}$, are zero in a neighborhood of the region where we formed the connected sum in our construction of hd $\left(D_{+}(K, t)\right)$. In particular, $n_{z^{\prime \prime}}(\phi)=n_{w^{\prime}}(\phi)=0$. Conversely, any holomorphic disk connecting $\mathbf{p}$ to $\mathbf{q}$ in $\widehat{C F}\left(S_{t}^{3}(K), \mathfrak{s}_{w^{\prime}}(\mathbf{p})\right)$ which satisfies $n_{z^{\prime \prime}}(\phi)=n_{w^{\prime}}(\phi)=0$ can be extended to a disk connecting $\left\{a_{1}\right\} \times \mathbf{p}$ to $\left\{a_{1}\right\} \times \mathbf{q}$. Thus the holomorphic disks that connect $\left\{a_{1}\right\} \times \mathbf{p}$ to $\left\{a_{1}\right\} \times \mathbf{q}$ for $\mathbf{p}, \mathbf{q}$ with $\mathfrak{s}_{w^{\prime}}(\mathbf{p})=\mathfrak{s}_{w^{\prime}}(\mathbf{q})$ correspond to holomorphic disks in $\widehat{C F}\left(S_{t}^{3}(K), \mathfrak{s}_{w^{\prime}}(\mathbf{p})\right)$ with $n_{z^{\prime \prime}}(\phi)=n_{w^{\prime}}(\phi)=0$ as claimed. (Here, the gluing theorem for pseudo-holomorphic disks used to prove stabilization invariance of Heegaard Floer homology is implicitly being used, [29, Section 11].)

\section{Computation of $\widehat{\operatorname{HFK}}\left(D_{+}(K, t), 1\right)$ for $|t| \gg 0$}

In this section we compute $\widehat{H F K}\left(D_{+}(K, t), 1\right)$ for all sufficiently large values of the twisting parameter, $t$. This is done by showing that $\bigoplus \widehat{H F K}\left(S_{t}^{3}(K), \mu_{K}, \mathfrak{s}_{i}\right)-$ which was identified with $\widehat{H F K}\left(D_{+}(K, t), 1\right)$ in the previous section - is determined by the filtered chain homotopy type of $C F K^{\infty}(K)$ in much the same way that $\widehat{H F}\left(S_{t}^{3}(K), \mathfrak{s}_{i}\right)$ is. (Recall that Ozsváth and Szabó present a formula for $\widehat{H F}\left(S_{t}^{3}(K), \mathfrak{s}_{i}\right)$ in terms of the filtered chain homotopy type of $C F K^{\infty}(K)$. This is summarized below but see [27] and also Ozsváth-Szabó [30; 31] for further details.)

Before stating the first result of the section, let us establish some notation regarding cobordisms and $\mathrm{Spin}^{c}$ structures. Let $W_{t}$ denote the cobordism from $S^{3}$ to $S_{t}^{3}(K)$ associated to the two-handle addition along $K$ with framing $t$ and $-W_{t}$ denote the same cobordism with reversed orientation. We can think of $-W_{t}$ as either a cobordism from $-S^{3}$ to $-S_{t}^{3}(K)$ or from $S_{t}^{3}(K)$ to $S^{3}$ - when we adopt the latter perspective 
we indicate it with a prime, $-W_{t}^{\prime}$. With these conventions, $\operatorname{Spin}^{c}$ structures on $S_{t}^{3}(K)$ $\left(\right.$ resp. $\left.S_{-t}^{3}(K)\right)$ can be labeled as follows: $\mathfrak{s}_{m} \in \operatorname{Spin}^{c}\left(S_{t}^{3}(K)\right)\left(\operatorname{resp} . \operatorname{Spin}^{c}\left(S_{-t}^{3}(K)\right)\right)$ is determined by the condition that it can be extended over the cobordism $-W_{t}^{\prime}$ (resp. $W_{-t}$ ) to yield a Spin ${ }^{c}$ structure $\mathfrak{r}_{m}$ satisfying:

$$
\left\langle c_{1}\left(\mathfrak{r}_{m}\right),[S]\right\rangle+t=2 m \quad\left(\operatorname{resp} .\left\langle c_{1}\left(\mathfrak{r}_{m}\right),[S]\right\rangle-t=2 m\right) .
$$

In terms of this labeling, we have the following Theorem.

Theorem 4.1 Let $K \subset S^{3}$ be a knot, and fix $m \in \mathbb{Z}$. Then there exists $T=T(m)>0$ such that for all $t>T$ the following holds:

$$
\begin{aligned}
\widehat{H F K}_{*}\left(S_{t}^{3}(K), \mu_{K}, \mathfrak{s}_{m}\right) \cong & H_{*+d_{-}(m)}(\mathcal{F}(K, m)) \oplus H_{*-2 m+d_{-}(m)}(\mathcal{F}(K,-m-1)) \\
\widehat{H F K}_{*}\left(S_{-t}^{3}(K), \mu_{K}, \mathfrak{s}_{m}\right) \cong & H_{*-d_{+}(m)}\left(\frac{\widehat{C F}\left(S^{3}\right)}{\mathcal{F}(K, m)}\right) \oplus \\
& H_{*-2 m-d_{+}(m)}\left(\frac{\widehat{C F}\left(S^{3}\right)}{\mathcal{F}(K,-m-1)}\right),
\end{aligned}
$$

where

$$
d_{ \pm}(m)=\left(\frac{t-(2 m \pm t)^{2}}{4 t}\right)
$$

Proof The theorem follows from an examination of [27, proof of Theorems 4.1 and 4.4]. Recall that these theorems yield isomorphisms for $t>T(m)$ :

$$
\begin{aligned}
\widehat{H F}_{*}\left(S_{t}^{3}(K), \mathfrak{s}_{m}\right) & \cong H_{*+d_{-}(m)}(C\{\max (i, j-m)=0\}) \\
\widehat{H F}_{*}\left(S_{-t}^{3}(K), \mu_{K}, \mathfrak{s}_{m}\right) & \cong H_{*-d_{+}(m)}(C\{\min (i, j-m)=0\}),
\end{aligned}
$$

where $C\{\max (i, j-m)=0\}$ denotes the subquotient complex of the $\mathbb{Z} \oplus \mathbb{Z}$ filtered chain complex, $C F K^{\infty}(K)$, generated by triples $[\mathbf{x}, i, j]$ with $i$ and $j$ filtration indices satisfying the specified constraint. The first isomorphism is induced by a chain map:

$$
\Phi_{\mathfrak{r}_{m}}: \widehat{C F}\left(S_{t}^{3}, \mathfrak{s}_{m}\right) \longrightarrow C\{\max (i, j-m)=0\},
$$

defined by counting pseudo-holomorphic triangles with appropriate boundary conditions in the $g$-fold symmetric product of $\Sigma_{g}$. The boundary conditions are determined by a doubly-pointed Heegaard triple diagram $(\Sigma, \boldsymbol{\alpha}, \boldsymbol{\beta}, \boldsymbol{\gamma}, w, z)$ specifying the 2handle cobordism $-W_{t}^{\prime}$. The three three-manifolds specified by the triple diagram are $Y_{\alpha, \beta}=S_{t}^{3}(K), Y_{\alpha, \gamma}=S^{3}, Y_{\beta, \gamma}=\#^{g-1} S^{1} \times S^{2}$ (see Figure 13). The map is defined by:

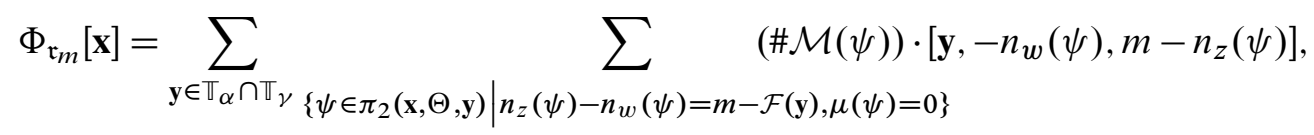


where $\Theta$ is a top-dimensional generator for $\widehat{H F}\left(\#^{g-1} S^{1} \times S^{2}\right)$. The second isomorphism is induced by a similar map which goes in the opposite direction. Since $n_{w}(\psi), n_{z}(\psi) \geq 0$ for holomorphic $\psi$, and the constraint on triples $[\mathbf{y}, i, j]$ generating $\operatorname{CFK}^{\infty}(K)$ is that

$$
\mathcal{F}(\mathbf{y})+(i-j)=0,
$$

it is clear that $\Phi_{\mathfrak{r}_{m}}$ maps into $C\{\max (i, j-m)=0\}$. Furthermore, the condition on the homotopy classes of triangles, $\psi$, in the above map ensures that the map indeed corresponds to $\mathfrak{r}_{m}$. To see this, recall from [29, Section 8] that a choice of basepoint determines a map:

$$
\mathfrak{s}_{w}: \pi_{2}(\mathbf{x}, \mathbf{y}, \mathbf{z}) \rightarrow \operatorname{Spin}^{c}\left(W_{\alpha, \beta, \gamma}\right),
$$

from homotopy classes of Whitney triangles $\psi \in \pi_{2}(\mathbf{x}, \mathbf{y}, \mathbf{z})$ to $\operatorname{Spin}^{c}$ structures on the four-manifold specified by the triple diagram. Further recall that the knot Floer homology filtration, $\mathcal{F}$, can be thought of as a filtration by $\operatorname{Spin}^{c}$ structures on $S_{0}^{3}(K)$. To make this correspondence, Ozsváth and Szabó assign to each intersection point $\mathbf{x}$ generating $\widehat{C F K}(K)$, an intersection point $\widetilde{\mathbf{x}}$ generating a chain complex, $\widehat{C F}\left(S_{0}^{3}(K)\right)$. The intersection point $\widetilde{\mathbf{x}}$ has an associated $\operatorname{Spin}^{c}$ structure, $\mathfrak{s}(\widetilde{\mathbf{x}}) \in \operatorname{Spin}^{c}\left(S_{0}^{3}(K)\right)$, which we denote $\mathfrak{s}(\mathbf{x}):=\mathfrak{s}(\widetilde{\mathbf{x}})$. Now the filtration of $\mathbf{x}$ is given by:

$$
\left\langle c_{1}(\underline{\mathfrak{g}}(\mathbf{x})),[\widehat{F}]\right\rangle:=2 \mathcal{F}(\mathbf{x}) .
$$

where $c_{1}(\underline{\mathfrak{s}}(\mathbf{x})) \in H^{2}\left(S_{0}^{3}(K), \mathbb{Z}\right) \cong \mathbb{Z}$ is the first Chern class of $\mathfrak{s}(\mathbf{x})$ and $[\widehat{F}] \in$ $H_{2}\left(S_{0}^{3}(K), \mathbb{Z}\right)$ is the homology class corresponding to a fixed Seifert surface of $K$, capped off by the meridian disk of the torus used in the zero-surgery. If we let $[S]$ denote the generator of $H_{2}\left(-W_{t}^{\prime}, \mathbb{Z}\right)$ we have the following analogue of [27, Equation 14]:

$$
\left\langle c_{1}(\underline{\mathfrak{s}}(\mathbf{x})),[\widehat{F}]\right\rangle+2\left(n_{z}(\psi)-n_{w}(\psi)\right)-t=\left\langle c_{1}\left(\mathfrak{s}_{w}(\psi)\right),[S]\right\rangle .
$$

With the definition of the filtration given above, it follows from Equation (6) that a homotopy class of triangles $\psi$ satisfying $n_{z}(\psi)-n_{w}(\psi)=m-\mathcal{F}(\mathbf{y})$ must also satisfy

$$
2 m-t=\left\langle c_{1}\left(\mathfrak{s}_{w}(\psi)\right),[S]\right\rangle,
$$

showing that $\Phi_{\mathfrak{r}_{m}}$ corresponds to $\mathfrak{r}_{m}$, as claimed.

We would like to refine Ozsváth and Szabó's theorem to determine the knot Floer homology of $\left(S_{t}^{3}(K), \mu_{K}\right)$. In fact, there is a natural sequence of subcomplexes:

$$
0 \subseteq C\{i<0, j=m\} \subseteq C\{\max (i, j-m)=0\} .
$$

Viewing this sequence as a 2-step filtration, we claim that its filtered chain homotopy type is equivalent to that of the filtration of $\widehat{C F}\left(S_{t}^{3}(K), \mathfrak{s}_{m}\right)$ induced by $\left(S_{t}^{3}(K), \mu_{K}\right)$. 
Theorem 4.1 will follow immediately, since

$$
\begin{aligned}
& C_{*}\{i<0, j=m\} \simeq \mathcal{F}_{*-2 m}(K,-m-1) \\
& \frac{C_{*}\{\max (i, j-m)=0\}}{C_{*}\{i<0, j=m\}} \simeq C_{*}\{i=0, j \leq m\} \simeq \mathcal{F}_{*}(K, m),
\end{aligned}
$$

by the discussion in [27, Section 3.5]. (Here $\simeq$ indicates chain homotopy equivalence.) The case when $t<0$ is similar.

The key observation in proving the claim is that the triple diagram $(\Sigma, \boldsymbol{\alpha}, \boldsymbol{\beta}, \boldsymbol{\gamma}, w, z)$ used to define $\Phi_{\mathfrak{r}_{m}}$ not only specifies a Heegaard diagram for the knot $\left(S^{3}, K\right)$ but also a diagram for $\left(S_{t}^{3}(K), \mu_{K}\right)$, with the addition of a basepoint, $z^{\prime}$. See Figure 13 , which illustrates the local picture of the Heegaard triple diagram near the basepoints Indeed, $\left(\Sigma, \boldsymbol{\alpha}, \boldsymbol{\beta}, w, z^{\prime}\right)$ is a diagram for $\left(S_{t}^{3}(K), \mu_{K}\right)$ as was noted in the discussion of the Heegaard diagram for the companion in Section 2.

Note Since this subsection deals exclusively with the surgery cobordism for the companion knot, $-W_{t}^{\prime}$, the notation here is independent from the previous two sections. In particular, to translate between the triple diagrams for $-W_{t}^{\prime}$ shown in Figure 13, Figure 14 and the similar-looking Heegaard diagrams for the companion, Figure 4 and Figure 11 we must first switch the roles of the $\alpha$ and $\beta$ curves on the triple diagram. Then we remove all $\gamma$ curves on the triple diagram except $\gamma_{g}$, which becomes the $\alpha$ curve $\mu_{K}$. Finally, we add a prime to each basepoint, so that $z \rightarrow z^{\prime}, w \rightarrow w^{\prime}, z^{\prime} \rightarrow z^{\prime \prime}$.

An intersection point $\mathbf{x} \in \mathbb{T}_{\alpha} \cap \mathbb{\mathbb { T }}_{\beta}$ is said to be supported in the winding region if the component of $\mathbf{x}$ in $\alpha_{g}$ lies in the local picture of Figure 13. Intersection points in the winding region are in $t$ to 1 correspondence with intersection points $\mathbf{y} \in \mathbb{T}_{\alpha} \cap \mathbb{T}_{\gamma}$. For $t$ sufficiently large, the pigeonhole principle shows that there exist an entire $\operatorname{Spin}^{c}$ equivalence class of intersection points for $\widehat{C F}\left(S_{t}^{3}(K)\right)$ supported in the winding region. Moreover, for fixed $m$ the equivalence class of intersection points corresponding to $\mathfrak{s}_{m}$ is supported in the winding region, provided that $t$ is large enough. This follows from Equation (6), which implies that the distance from $x_{0} \in \alpha_{g} \cap \gamma_{g}$ to the $\beta_{g}$ component of a point $\mathbf{x}$ supported in the winding region is proportional to $|m|$ for the $\operatorname{Spin}^{c}$ structure, $\mathfrak{s}_{m}$, which it represents.

Now the generators of the equivalence class corresponding to $\mathfrak{s}_{m}$ are in bijection with generators of $C\{\max (i, j-m)=0\}$. As shown by Ozsváth and Szabó, this bijection is induced by canonical "small" triangles supported entirely in the winding region and which connect generators of the two complexes, see Figure 13. In fact the pseudo-holomorphic representatives of these triangles constitute the highest order 


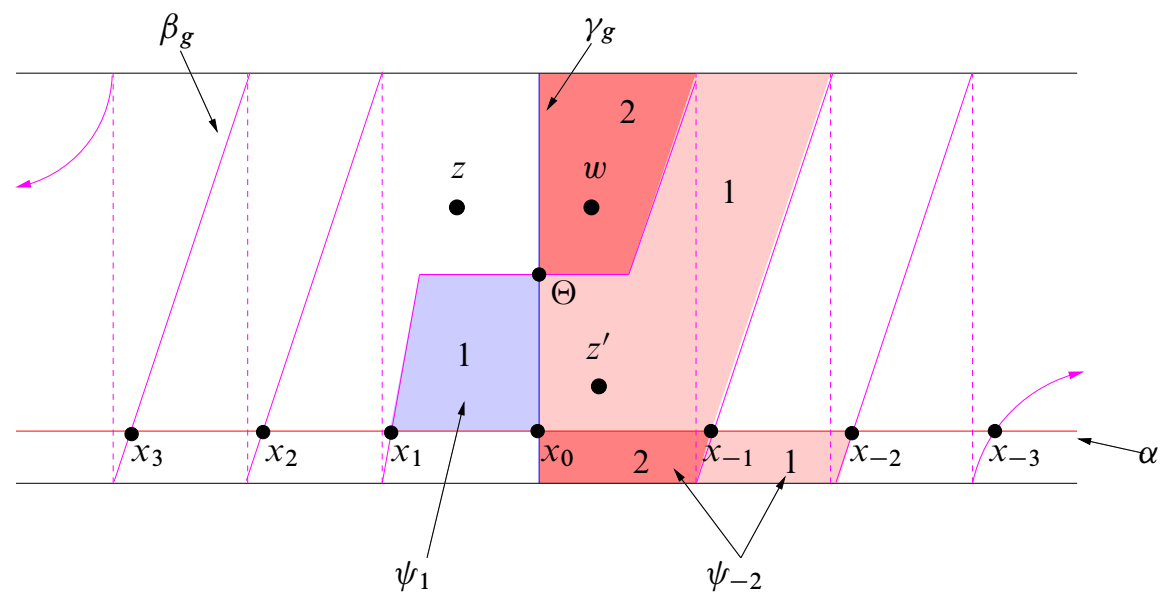

Figure 13: Heegaard triple for $-W_{t}^{\prime}$ near the basepoints (here $t=6$ ). We refer to this part of the Heegaard diagram as the "winding region". To the left of $\gamma_{g}$, the domain of a small triangle $\psi_{1}$ connecting $x_{1}$ to $x_{0}$ is shaded (in blue). A small triangle $\psi_{-2}$ connecting $x_{-2}$ to $x_{0}$, with $n_{w}\left(\psi_{-2}\right)=2$ is shaded (in red) to the right of $\gamma_{g}$. Note that while the picture looks like the diagram for hd $\left(D_{+}(K, t)\right)$ near the connect sum region, this is a Heegaard triple diagram, and for the moment we make no reference to the diagram for the Whitehead doubles. Thus the notation here is independent of the previous two sections.

terms of $\Phi_{\mathfrak{r}_{m}}$ (with respect to the filtration given by negative area of triangles), and subsequently induce the isomorphism on homology given by Equation (3).

From Figure 13 we see that there is a unique intersection point $x_{0} \in \alpha_{g} \cap \gamma_{g}$. The multiplicity of each small triangle at the basepoints measures how far to the right or left of $x_{0}$ the $\alpha_{g}$ component of the corresponding generator of $\widehat{C F K}\left(S_{t}^{3}(K), \mu_{K}, \mathfrak{s}_{m}\right)$ lies. Suppose $\psi \in \pi_{2}(\mathbf{x}, \Theta, \mathbf{y})$ is a small triangle. If $k=n_{z}(\psi) \geq 0$ then the $\alpha_{g}$ component of $\mathbf{x}$ is $x_{k}$, and $[\mathbf{x}]$ is mapped by $\Phi_{\mathfrak{r}_{m}}$ to the quotient complex, $C\{0, j \leq m\}$, of $C\{\max (i, j-m)=0\}$. If, on the other hand, $l=n_{w}(\psi)>0$ the $\alpha_{g}$ component of $\mathbf{x}$ is $x_{-l}$ and $[\mathbf{x}]$ is mapped to the subcomplex, $C\{i<0, j=m\}$.

Lemma 4.2 Let $\mathbf{p}, \mathbf{q} \in \widehat{C F K}\left(S_{t}^{3}(K), \mu_{K}, \mathfrak{s}_{m}\right)$ be two generators supported in the winding region, and let $x_{i}, x_{j}$ denote the $\alpha_{g}$ components of the corresponding intersection points in $\mathbb{T}_{\alpha} \cap \mathbb{\mathbb { T }}_{\beta}$. Then

$$
\mathcal{F}(\mathbf{p})-\mathcal{F}(\mathbf{q})= \begin{cases} \pm 1 & \text { if } x_{i}, x_{j} \text { lie on opposite sides of } x_{0} \\ 0 & \text { otherwise }\end{cases}
$$


Proof Note that in terms of Figure 13, $x_{i}, x_{j}$ lie on opposite sides of $x_{0}$ precisely when $i$ and $j$ have opposite signs. We will construct a Whitney disk, $\phi_{\mathbf{p}, \mathbf{q}} \in \pi_{2}(\mathbf{p}, \mathbf{q})$, which has the following two properties:

(1) The boundary of the domain of $\phi_{\mathbf{p}, \mathbf{q}}$ contains the arc, $\delta$, connecting $x_{1}$ and $x_{-1}$ on $\beta_{g}$ if and only if $x_{i}, x_{j}$ lie on opposite sides of $x_{0}$

(2) $\# \delta \subset \partial \phi_{\mathbf{p}, \mathbf{q}}=1$ if $x_{i}$ and $x_{j}$ are on opposite sides of $x_{0}$, and is 0 otherwise.

See Figure 14. Assuming the existence of such $\phi_{\mathbf{p}, \mathbf{q}}$, the lemma follows immediately from the definition of the filtration of $\widehat{C F K}\left(S_{t}^{3}(K), \mu_{K}, \mathfrak{s}_{m}\right)$.

To construct $\phi_{\mathbf{p}, \mathbf{q}}$, note first that since $\mathbf{p}, \mathbf{q}$ lie in the winding region, they correspond uniquely to intersection points $\widetilde{\mathbf{p}}, \widetilde{\mathbf{q}} \in \mathbb{T}_{\alpha} \cap \mathbb{T}_{\gamma}$. These intersection points can be connected by a Whitney disk $\phi$ with $n_{w}(\phi)=0$ and $n_{z}(\phi)=k$ for some $k \in \mathbb{Z}$. This means that $\partial \phi$ contains $\gamma_{g}$ with multiplicity $k$, which further implies that the distance between $x_{i}, x_{j}$ is $k$, ie $i-j=k$. The domain of $\phi_{\mathbf{p}, \mathbf{q}}$ can then be obtained from the domain of $\phi$ by a simple modification in the winding region. This modification is shown in Figure 14. It replaces the boundary component $k \cdot \gamma_{g} \subset \partial \phi$ by a simple closed curve formed from an arc connecting $x_{i}$ to $x_{j}$ along $\alpha_{g}$ followed by an arc connecting $x_{j}$ to $x_{i}$ along $\beta_{g}$, and which wraps $k$ times around the neck of the winding region. The enumerated properties of $\partial \phi_{\mathbf{p}, \mathbf{q}}$ are immediate.

The identification of the 2-step filtration of $C\{\max (i, j-m)=0\}$ with the filtration induced by $\left(S_{t}^{3}(K), \mu_{K}\right)$ follows from the lemma and the paragraph preceding it. Explicitly, under the bijection between generators induced by the small triangles, we have the following identifications (here $\mathbf{x} \in \widehat{C F K}\left(S_{t}^{3}(K), \mu_{K}, \mathfrak{s}_{m}\right)$ is a point supported in the winding region):

$\mathbf{x}$ with $\alpha_{g}$ component lying to the left of $x_{0} \leftrightarrow C\{i=0, j \leq m\}$

$\mathbf{x}$ with $\alpha_{g}$ component lying to the right of $x_{0} \leftrightarrow C\{i<0, j=m\}$.

The lemma identifies generators to the left of $x_{0}$ with the top filtration level of $\widehat{C F K}\left(S_{t}^{3}(K), \mu_{K}, \mathfrak{s}_{m}\right)$ and those to the right of $x_{0}$ with the bottom filtration level. This completes the proof of Theorem 4.1.

We have only shown that for fixed $m$, there exists a framing large enough so that $\widehat{H F K}_{*}\left(S_{t}^{3}(K), \mu_{K}, \mathfrak{s}_{m}\right)$ is given by the formula of Theorem 4.1. Since the Floer homology of the Whitehead double involves the sum $\bigoplus_{m} \widehat{H F K} \widehat{H}_{*}\left(S_{t}^{3}(K), \mu_{K}, \mathfrak{s}_{m}\right)$, it will be necessary to prove the following refinement. 

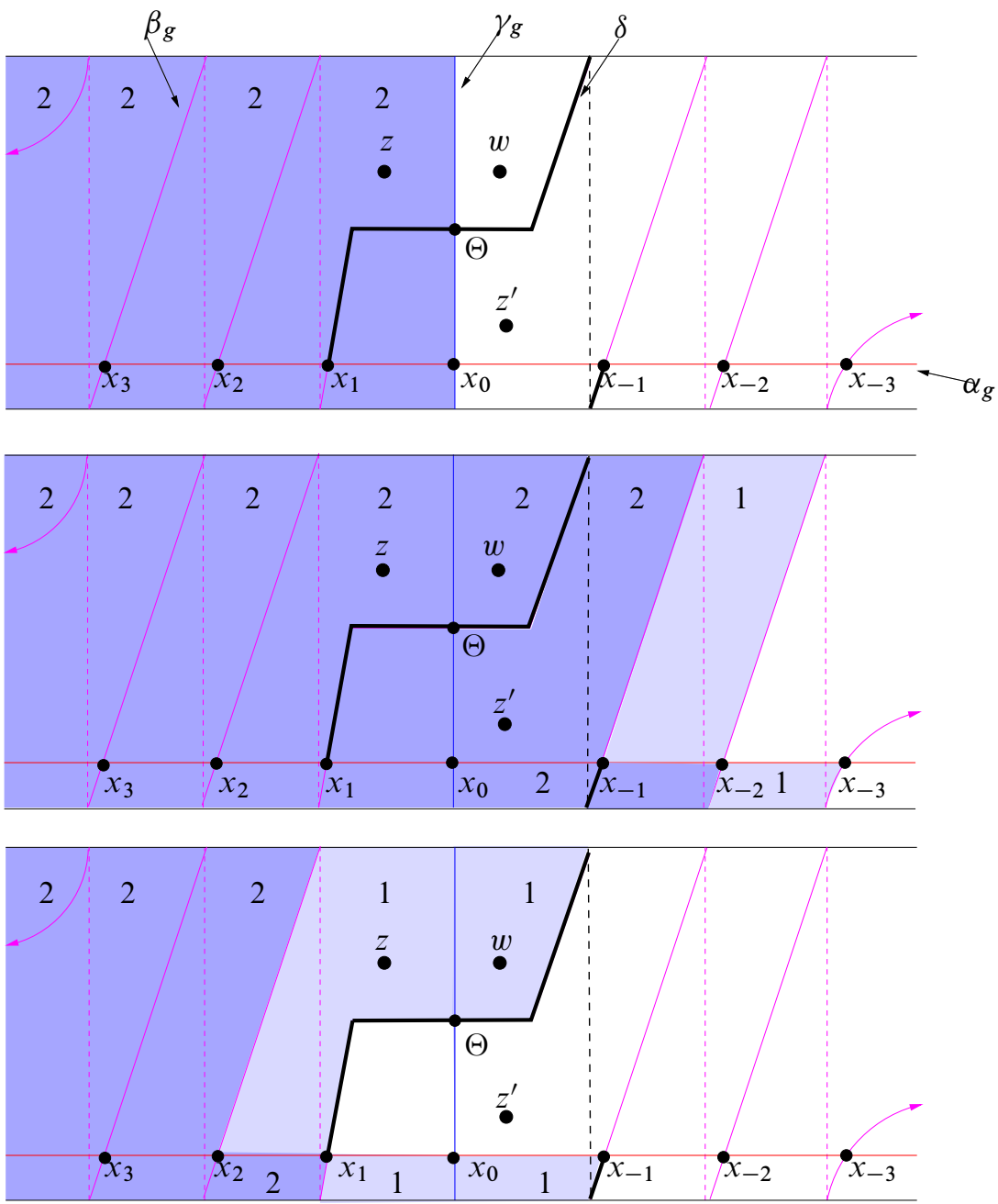

Figure 14: Figure illustrating the process by which the domain of a disk $\phi_{\mathbf{p}, \mathbf{q}} \in \pi_{2}(\mathbf{p}, \mathbf{q})$, for $\mathbf{p}, \mathbf{q} \in \mathbb{T}_{\alpha} \cap \mathbb{T}_{\beta}$ in the winding region can be identified with the domain of a disk, $\phi \in \pi_{2}(\widetilde{\mathbf{p}}, \widetilde{\mathbf{q}})$. Here $\widetilde{\mathbf{p}}, \widetilde{\mathbf{q}}$ are the intersection points in $\mathbb{T}_{\alpha} \cap \mathbb{T}_{\gamma}$ corresponding to $\mathbf{p}, \mathbf{q}$ under the $t$ to 1 identification of points supported in the winding region with points in $\mathbb{T}_{\alpha} \cap \mathbb{T}_{\gamma}$. Note that the multiplicity with which the arc, $\delta$, occurs in $\partial \phi_{\mathbf{p}, \mathbf{q}}$ is \pm 1 if the $\alpha_{g}$ components of $\mathbf{p}, \mathbf{q}$ lie on opposite sides of $x_{0}$, and 0 otherwise. 

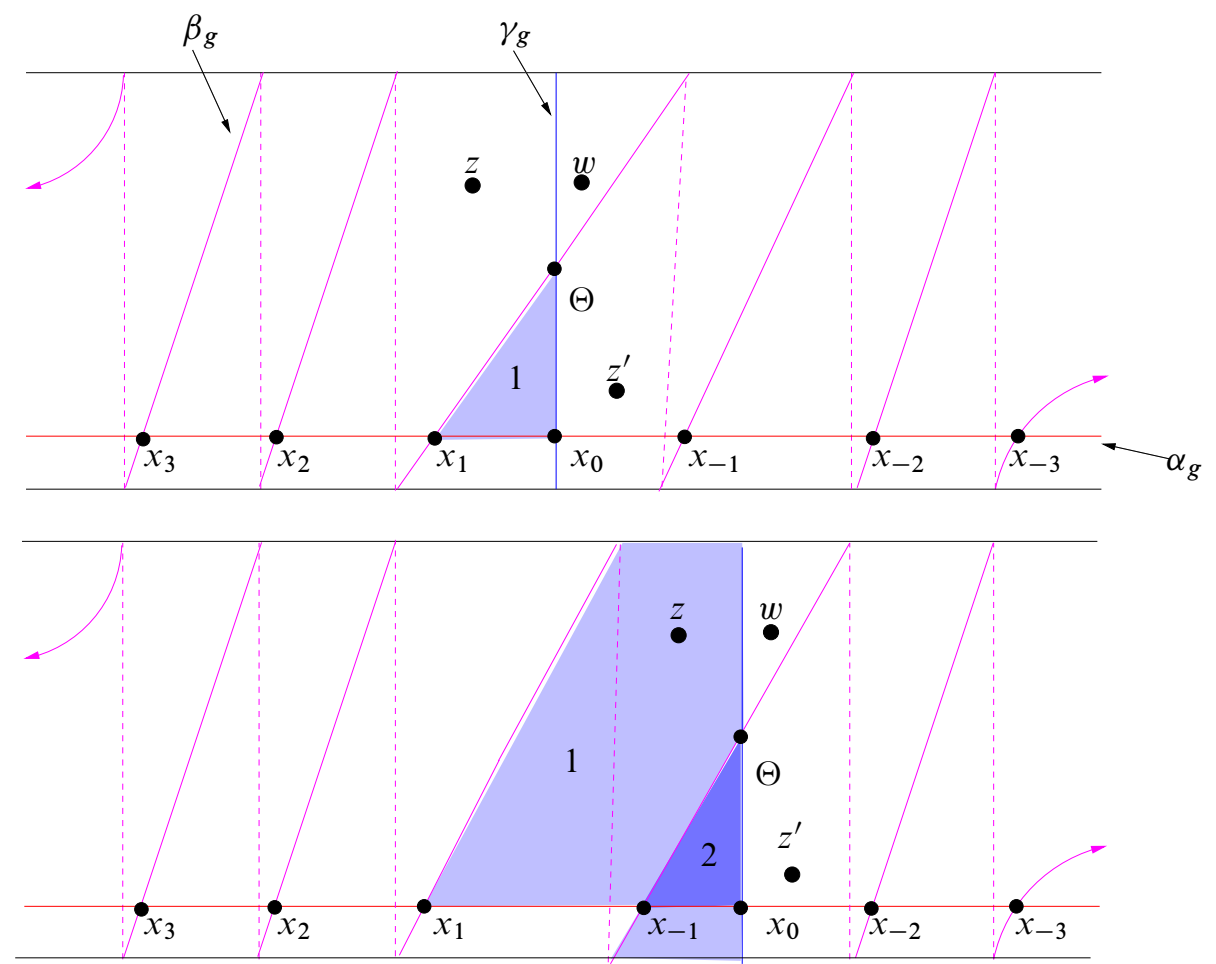

Figure 15: Procedure by which we vary the placement of the quadruple $\left(\gamma_{g}, z, w, z^{\prime}\right)$ to arrange that a single $\operatorname{Spin}^{c}$ equivalence class of intersection points supported in the winding region can be used to calculate $\widehat{H F K}_{*}\left(S_{ \pm t}^{3}(K), \mu_{K}, \mathfrak{s}_{m}\right)$ for all $\mathfrak{s}_{m}$. Note that the small triangle connecting an intersection point, eg $\left\{x_{1}, \mathbf{x}\right\}$, in our equivalence class is modified upon moving the basepoint. This implies, by Equation (6) and [24, Proposition 6.3], that the $\operatorname{Spin}^{c}$ structure represented by our equivalence class changes as described in the text.

Theorem 4.3 Let $K \subset S^{3}$ be a knot. Then there exists $T>0$ such that for all $t>T$ the following holds for all $\mathrm{m}$ :

$\widehat{H F K}_{*}\left(S_{t}^{3}(K), \mu_{K}, \mathfrak{s}_{m}\right) \cong H_{*+d_{-}(m)}(\mathcal{F}(K, m)) \oplus H_{*-2 m+d_{-}(m)}(\mathcal{F}(K,-m-1))$

$\widehat{H F K}_{*}\left(S_{-t}^{3}(K), \mu_{K}, \mathfrak{s}_{m}\right) \cong H_{*-d_{+}(m)}\left(\frac{\widehat{C F}\left(S^{3}\right)}{\mathcal{F}(K, m)}\right) \oplus H_{*-2 m-d_{+}(m)}\left(\frac{\widehat{C F}\left(S^{3}\right)}{\mathcal{F}(K,-m-1)}\right)$.

Proof The refinement is proved by the somewhat standard technique of "moving the basepoint," see Ozsváth-Szabó [28] and Rasmussen [32]. In the present context, this has the following meaning: in the proof of Theorem 4.1, the framing parameter was 
increased so that the $\operatorname{Spin}^{c}$-equivalence class of intersection points corresponding to $\mathfrak{s}_{m} \in \operatorname{Spin}^{c}\left(S_{t}^{3}(K)\right)$ was represented by intersection points supported in the winding region. In the Heegaard diagram, however, there were a number of $\operatorname{Spin}^{c}$-equivalence classes which contained intersection points outside the winding region. By moving the quadruple of data $\left(\gamma_{g}, z, w, z^{\prime}\right)$ one intersection point to the right of $x_{-1}$ (see Figure 15), the $\operatorname{Spin}^{c}$-equivalence class of intersection points with which we started no longer corresponds to $\mathfrak{s}_{m}$. Instead, it represents $\mathfrak{s}_{m+1}$, as can be seen (for instance) from Equation (6), together with the formula for the Chern class of the $\operatorname{Spin}^{c}$ structure associated to a Whitney triangle, Ozsváth-Szabó [24, Proposition 6.3]. Moving $\left(\gamma_{g}, z, w, z^{\prime}\right)$ to the left of $x_{1}$ changes the $\operatorname{Spin}^{c}$ structure to $\mathfrak{s}_{m-1}$. Despite the change in location of $\gamma_{g}$, there are still small triangles in the winding region that connect each intersection point in our equivalence class to the corresponding intersection point in $\mathbb{T}_{\alpha} \cap \mathbb{\mathbb { V }}_{\gamma}$. Thus Theorem 4.1 applies, with the same $T(m)$, to yield the result for $\mathfrak{s}_{m-1}, \mathfrak{s}_{m+1}$. Varying the placement of the meridian and basepoints throughout the winding region proves the theorem for all $\mathfrak{s} \in \operatorname{Spin}^{c}\left(S_{t}^{3}(K)\right)$.

\subsection{Gradings}

We conclude this section by determining, when $|t| \gg 0$, the absolute Maslov grading of $\widehat{H F K}\left(D_{+}(K, t), 1\right)$. We show the following Theorem.

Theorem 4.4 With notation as above, there are isomorphisms of absolutely $\mathbb{Z}$-graded abelian groups for all $t>T$ :

$$
\begin{gathered}
\widehat{H F K}_{*}\left(D_{+}(K, t), 1\right) \cong \bigoplus_{\substack{m=\left\lfloor-\frac{t}{2}\right\rfloor+1 \\
m=\left\lfloor\frac{t}{2}\right\rfloor}}^{m=\left\lfloor\frac{t}{2}\right\rfloor}\left[H_{*-1}(\mathcal{F}(K, m)) \oplus H_{*-1}(\mathcal{F}(K,-m-1))\right] \\
\left.\widehat{H F K}_{*}\left(D_{+}(K,-t), 1\right) \cong \bigoplus_{m=\left\lfloor-\frac{t}{2}\right\rfloor+1}\left(\frac{\widehat{C F}\left(S^{3}\right)}{\mathcal{F}(K, m)}\right) \oplus H_{*}\left(\frac{\widehat{C F}\left(S^{3}\right)}{\mathcal{F}(K,-m-1)}\right)\right] .
\end{gathered}
$$

Proof By a sequence of Heegaard moves, each of which occur in the complement of the basepoint $w$, we can convert hd $\left(D_{+}(K, t)\right)$ to hd $(K)$. See Figure 7. Since these moves occur in the complement of $w$, the absolute grading of any generators unaffected by the Heegaard moves is unchanged throughout the process. It follows that intersection points of the form:

$$
\{y\} \times \mathbf{p}_{i}, \in \widehat{C F K}(P) \times \widehat{C F K}(K)
$$

inherit the grading which $\mathbf{p}_{i}$ has, thought of as a generator of $\widehat{C F K}(K)$. As mentioned in the proof of Theorem 4.1 above, intersection points $\mathbf{p}_{i} \in \widehat{C F K}(K)$ are in 1 to 
$t$ correspondence with intersection points in the winding region, and hence with intersection points of the form:

$$
\left\{a_{j}\right\} \times \mathbf{q}_{i} \in\left\{a_{j}\right\} \times \widehat{C F}\left(S_{t}^{3}(K)\right),
$$

where $\mathbf{q}_{i}$ is supported in the winding region. More explicitly, each point of $\{y\} \times$ $\widehat{C F K}(K)$ is of the form $\{y\} \times\left\{x_{0}, \mathbf{p}\right\}$, for some $(g-1)$-tuple of intersection points, $\mathbf{p}$, while each point of $\left\{a_{j}\right\} \times \widehat{C F}\left(S_{t}^{3}(K)\right)$ in the winding region is of the form $\left\{a_{j}\right\} \times\left\{x_{k}, \mathbf{p}\right\}$ for some $x_{k} \in \beta_{g} \cap \lambda_{K} \# \lambda_{P}$, where $k=\left\lfloor-\frac{t}{2}\right\rfloor+1, \ldots,\left\lfloor\frac{t}{2}\right\rfloor$. In order to determine the absolute gradings for the Floer homology of the Whitehead double, we first understand the grading on the intersection points supported in the winding region. These points are partitioned into two groups - those points whose $\beta_{g}$ component is to the left of $x_{0}$ (those with $k>0$ ), and those whose $\beta_{g}$ component is to the right $(k<0)$. We first handle points to the left of $x_{0}$.

Lemma 4.5 Let $k>0$. Then for $t \gg 0$ we have

$$
\operatorname{gr}\left(\left\{a_{1}\right\} \times\left\{x_{k}, \mathbf{p}\right\}\right)=\operatorname{gr}\left(\{y\} \times\left\{x_{0}, \mathbf{p}\right\}\right)+1,
$$

while for $t \ll 0$,

$$
\operatorname{gr}\left(\left\{a_{1}\right\} \times\left\{x_{k}, \mathbf{p}\right\}\right)=\operatorname{gr}\left(\{y\} \times\left\{x_{0}, \mathbf{p}\right\}\right) .
$$

Proof For $k>0$, we can complete the small triangles connecting $x_{k}$ to $x_{0}$ used in the proof of Theorem 4.1 with domains on the diagram for $P$ to obtain domains of Whitney disks. This is shown in Figure 11 for $t<0$ and Figure 12 for $t>0$. For $t>0$ the Whitney disk $\phi_{a_{1}, y}$ connects $\left\{a_{1}\right\} \times\left\{x_{k}, \mathbf{p}\right\}$ to $\{y\} \times\left\{x_{0}, \mathbf{p}\right\}$. For $t<0$ we have a Whitney disk $\phi_{y, a_{4}}$ connecting $\{y\} \times\left\{x_{0}, \mathbf{p}\right\}$ to $\left\{a_{4}\right\} \times\left\{x_{k}, \mathbf{p}\right\}$. Section 3.1 calculated $\mu\left(\phi_{a_{1}, y}\right)=\mu\left(\phi_{y, a_{4}}\right)=1$. For $t \gg 0$, the Lemma follows from the formula for the absolute grading:

$$
\operatorname{gr}\left(\left\{a_{1}\right\} \times\left\{x_{k}, \mathbf{p}\right\}\right)-\operatorname{gr}\left(\{y\} \times\left\{x_{0}, \mathbf{p}\right\}\right)=\mu\left(\phi_{a_{1}, y}\right)-2 n_{w}\left(\phi_{a_{1}, y}\right)=1 .
$$

For $t \ll 0$, we have

$$
\operatorname{gr}\left(\{y\} \times\left\{x_{0}, \mathbf{p}\right\}\right)-\operatorname{gr}\left(\left\{a_{4}\right\} \times\left\{x_{k}, \mathbf{p}\right\}\right)=\mu\left(\phi_{y, a_{4}}\right)-2 n_{w}\left(\phi_{a_{1}, y}\right)=1,
$$

and

$$
\operatorname{gr}\left(\left\{a_{1}\right\} \times\left\{x_{k}, \mathbf{p}\right\}\right)-\operatorname{gr}\left(\left\{a_{4}\right\} \times\left\{x_{k}, \mathbf{p}\right\}\right)=\mu\left(\phi_{a_{1}, a_{4}}\right)-2 n_{w}\left(\phi_{a_{1}, a_{4}}\right)=1,
$$

where $\phi_{a_{1}, a_{4}}$ is one of the four Whitney disks with annular domains discussed in the proof of Lemma 3.4. The lemma follows from the additivity of the grading. 
Next we deal with the points to the right of $x_{0}$. Before stating the lemma, we remark that each generator of $\widehat{C F K}(K)$ is of the form $x_{0} \times \mathbf{p}$, and hence we can think of the $(g-1)$-tuple $\mathbf{p}$ as having a filtration, $\mathcal{F}(\mathbf{p})$ inherited from the filtration of $\widehat{C F K}(K)$. Note also that each point in the winding region $x_{k} \times \mathbf{p}$ uniquely corresponds to an intersection point $x_{0} \times \mathbf{p} \in \widehat{C F K}(K)$.

Lemma 4.6 Let $-k<0$. Then for $t \gg 0$ we have

$$
\operatorname{gr}\left(\left\{a_{1}\right\} \times\left\{x_{-k}, \mathbf{p}\right\}\right)=\operatorname{gr}\left(\{y\} \times\left\{x_{0}, \mathbf{p}\right\}\right)+2 \mathcal{F}(\mathbf{p})+1,
$$

while for $t \ll 0$,

$$
\operatorname{gr}\left(\left\{a_{1}\right\} \times\left\{x_{-k}, \mathbf{p}\right\}\right)=\operatorname{gr}\left(\{y\} \times\left\{x_{0}, \mathbf{p}\right\}\right)+2 \mathcal{F}(\mathbf{p}) .
$$

Proof We handle only the second case, as the first is similar. We would like to complete the small triangle $\psi_{-k}$ connecting $\left\{x_{-k}, \mathbf{p}\right\}$ to $\left\{x_{0}, \mathbf{p}\right\}$ by a domain on $h d(P)$, as in Figure 11. However, since the small triangle is now supported to the right of $x_{0}$, the multiplicities of the domain of the triangle near the connect sum region (the black hole) are not suitable for completion, see Figure 16. Thus we pick a homotopy class of triangles connecting $\left\{x_{-k}, \mathbf{p}\right\}$ to $\left\{x_{0}, \mathbf{p}\right\}$ which has multiplicity 0 in the two domains on the right of the connect sum tube. This homotopy class is given by

$$
\psi_{-k}^{\prime}=\psi_{-k}-k \cdot[\Sigma]-\mathcal{P}^{3},
$$

where $[\Sigma]$ is the domain corresponding to the homology class of the Heegaard surface and $\mathcal{P}^{3}$ is the generator of the space of triply periodic domains. The domain of $\psi_{-k}^{\prime}$ is illustrated in Figure 16. Since the multiplicities of $\psi_{-k}^{\prime}$ are the same near the connect sum region as the small triangles used in the previous lemma, it can be completed on hd $(P)$ to the domain of a Whitney disk $\phi \in \pi_{2}\left(\{y\} \times\left\{x_{0}, \mathbf{p}\right\},\left\{a_{4}\right\} \times\left\{x_{k}, \mathbf{p}\right\}\right)$, as in Figure 11. As with the previous lemma, the Maslov index of $\phi$ will be one higher than that of the triangle used to construct the domain:

$$
\mu(\phi)=\mu\left(\psi_{-k}^{\prime}\right)+1 .
$$

(This calculation follows in the same spirit as the calculation of the Maslov indices in Section 3.1). Assuming, for the moment, that $\mu\left(\psi_{-k}^{\prime}\right)=-2 \mathcal{F}(\mathbf{p})$, the second part of the lemma follows. For we have:

$$
\begin{gathered}
\operatorname{gr}\left(\{y\} \times\left\{x_{0}, \mathbf{p}\right\}\right)-\operatorname{gr}\left(\left\{a_{4}\right\} \times\left\{x_{k}, \mathbf{p}\right\}\right)=\mu(\phi)-2 n_{w}(\phi)=-2 \mathcal{F}(\mathbf{p})+1, \\
\operatorname{gr}\left(\left\{a_{1}\right\} \times\left\{x_{k}, \mathbf{p}\right\}\right)-\operatorname{gr}\left(\left\{a_{4}\right\} \times\left\{x_{k}, \mathbf{p}\right\}\right)=\mu\left(\phi_{a_{1}, a_{4}}\right)-2 n_{w}\left(\phi_{a_{1}, a_{4}}\right)=1,
\end{gathered}
$$

which together yield the second part of the lemma. Since both the procedure for constructing a disk $\phi$ and the calculation of $\mu(\phi)$ are the same for $t \gg 0$ we leave the first part of the lemma for the reader. Thus it will suffice to verify $\mu\left(\psi_{-k}^{\prime}\right)=-2 \mathcal{F}(\mathbf{p})$. 
First note the effect of adding the domain of the Heegaard surface:

$$
\mu\left(\psi_{-k}-k \cdot[\Sigma]\right)=\mu\left(\psi_{-k}\right)-k \cdot \mu([\Sigma])=\mu\left(\psi_{-k}\right)-2 k .
$$

The effect of subtracting $\mathcal{P}^{3}$ can be determined from the definition of the absolute grading of Floer homology for torsion $\operatorname{Spin}^{c}$ structures, [24, Equation 12]:

$$
\mu\left(\psi-\mathcal{P}^{3}\right)-\mu(\psi)=\frac{1}{4}\left(\left(c_{1}\left(\mathfrak{s}_{w}\left(\psi-\mathcal{P}^{3}\right)\right)^{2}-\left(c_{1}\left(\mathfrak{s}_{w}(\psi)\right)^{2}\right) .\right.\right.
$$

In the above equation, $\mathfrak{s}_{w}(\psi)$ denotes the $\operatorname{Spin}^{c}$ structure on the two-handle cobordism $-W_{t}^{\prime}$ associated to the triangle $\psi$ via the basepoint $w$, and $c_{1}$ denotes its first Chern class. Now for an arbitrary homotopy class of triangles, $\psi$, Equations (5) and (6) together imply:

$$
2 \mathcal{F}(\mathbf{p})+2\left(n_{z}(\psi)-n_{w}(\psi)\right)-t=\left\langle c_{1}\left(\mathfrak{s}_{w}(\psi)\right),[S]\right\rangle,
$$

and hence for the small triangle $\psi_{-k}$ this becomes:

$$
2 \mathcal{F}(\mathbf{p})-2 k-t=\left\langle c_{1}\left(\mathfrak{s}_{w}\left(\psi_{-k}\right)\right),[S]\right\rangle .
$$

Subtracting $\mathcal{P}^{3}$ from $\psi_{-k}$ changes the $\operatorname{Spin}^{c}$ structure associated to the triangle:

$\left\langle c_{1}\left(\mathfrak{s}_{w}\left(\psi_{-k}-\mathcal{P}^{3}\right)\right),[S]\right\rangle=\left\langle c_{1}\left(\mathfrak{s}_{w}\left(\psi_{-k}\right)\right)-2 \operatorname{PD}[S],[S]\right\rangle=\left\langle c_{1}\left(\mathfrak{s}_{w}\left(\psi_{-k}\right)\right),[S]\right\rangle+2 t$.

We can now compute $c_{1}^{2}$ for the $\operatorname{Spin}^{c}$ structures associated to $\psi_{-k}$ and $\psi_{-k}-\mathcal{P}^{3}$ and determine the difference in their Maslov indices using Equation (7):

$\mu\left(\psi_{-k}-\mathcal{P}^{3}\right)-\mu\left(\psi_{-k}\right)=\frac{1}{4}\left[\frac{(2 \mathcal{F}(\mathbf{p})-2 k+t)^{2}}{-t}-\frac{(2 \mathcal{F}(\mathbf{p})-2 k-t)^{2}}{-t}\right]=2(k-\mathcal{F}(\mathbf{p}))$.

Thus, the Maslov index of $\psi_{-k}^{\prime}$ is given by:

$$
\mu\left(\psi_{-k}-k \cdot[\Sigma]-\mathcal{P}^{3}\right)=\mu\left(\psi_{-k}-\mathcal{P}^{3}\right)-2 k=2(k-\mathcal{F}(\mathbf{p}))-2 k=-2 \mathcal{F}(\mathbf{p}),
$$

as claimed.

For intersection points generating $\widehat{H F K}\left(D_{+}(K, t), 1\right)$ which are supported in the winding region, the two lemmas above are enough to complete the proof of Theorem 4.4. More explicitly, the isomorphism in Theorem 4.1,

$\widehat{H F K}_{*}\left(S_{t}^{3}(K), \mu_{K}, \mathfrak{s}_{m}\right) \cong H_{*+d_{-}(m)}(\mathcal{F}(K, m)) \oplus H_{*-2 m+d_{-}(m)}(\mathcal{F}(K,-m-1))$,

was proved by looking at intersection points in the winding region, with the $\mathcal{F}(K, m)$ summand corresponding to those $\left\{x_{k}, \mathbf{p}\right\} \in \widehat{C F K}\left(S_{t}^{3}(K)\right)$ with $x_{k}$ to the left of $x_{0}$, and $\mathcal{F}(\mathbf{p}) \leq m$. By Lemma 4.5 and the discussion at the beginning of the proof of this theorem, the grading of the corresponding intersection points $\left\{a_{1}\right\} \times\left\{x_{k}, \mathbf{p}\right\} \in$ $\widehat{C F K}\left(D_{+}(K, t), 1\right)$ is the same as the grading of $\left\{x_{0}, \mathbf{p}\right\}$ (or shifted up by 1 , depending 

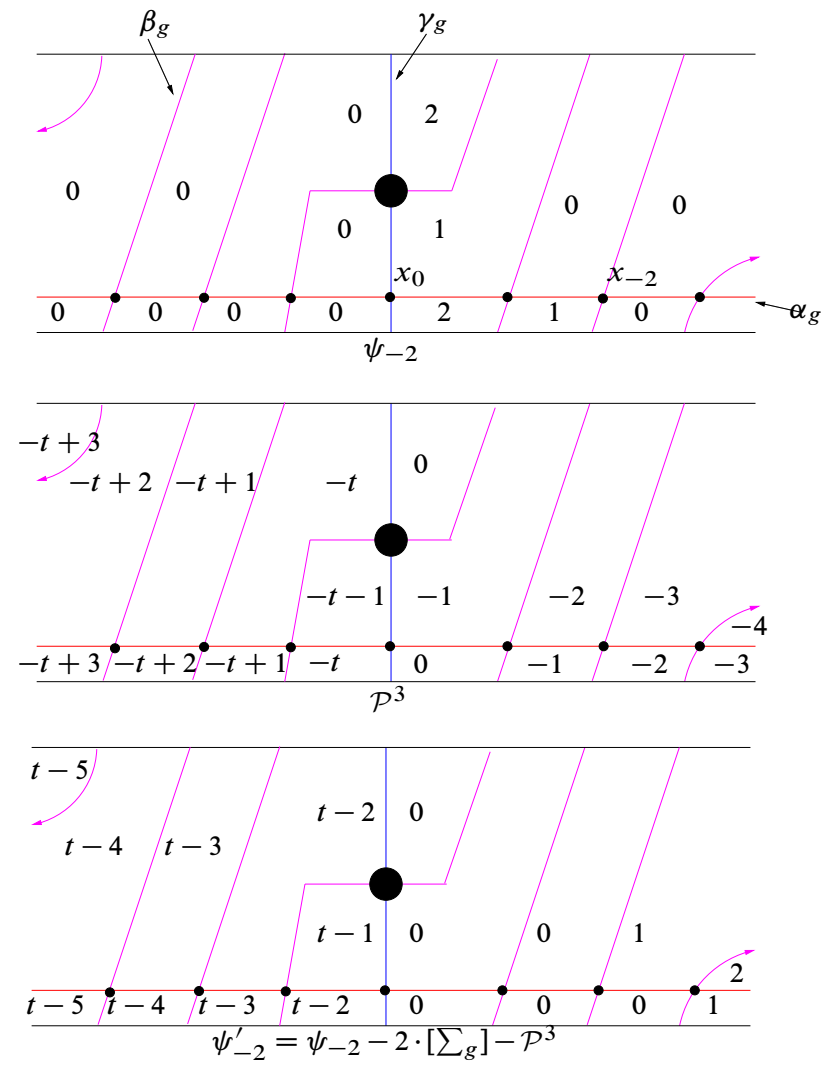

Figure 16: Illustration for Lemma 4.6. The first part of the Figure depicts multiplicities of the small triangle $\psi_{-2}$. The second shows multiplicities of the generator of the space of triply-periodic domains, $\mathcal{P}^{3}$. The last part shows multiplicities of $\psi_{-2}^{\prime}=\psi_{-2}-2 \cdot\left[\Sigma_{g}\right]-\mathcal{P}^{3}$. The domain of $\psi_{-2}^{\prime}$ can be completed to the domain of a Whitney disk on the Heegaard diagram for the Whitehead double, as in Figure 11. The case with $t>0$ is analogous.

on whether $t$ is positive or negative). Hence the homology class $\left[\left\{a_{1}\right\} \times\left\{x_{k}, \mathbf{p}\right\}\right] \in$ $\widehat{C F K}\left(D_{+}(K, t), 1\right)$ inherits its grading from $H_{*}(\mathcal{F}(K, m))$. The correction factor $d_{-}(m)$ is missing since the Whitehead double is a knot in $S^{3}$ and $d_{-}(m)$ came from the grading of $S_{t}^{3}(K)$.

On the other hand, the $\mathcal{F}(K,-m-1)$ summand in Theorem 4.4 corresponds to those $\left\{a_{1}\right\} \times\left\{x_{-k}, \mathbf{p}\right\} \in \widehat{C F K}($ Hopf $) \times \widehat{C F K}\left(S_{t}^{3}(K)\right)$ with $x_{-k}$ to the right of $x_{0}$, and $\mathcal{F}(\mathbf{p})>m$. Pick $\left\{a_{1}\right\} \times\left\{x_{-k}, \mathbf{p}\right\}$. We wish to understand the induced grading of $\left[\left\{a_{1}\right\} \times\left\{x_{-k}, \mathbf{p}\right\}\right] \in \widehat{H F K}\left(D_{+}(K, t), 1\right)$. By Lemma 4.6 and the discussion at the 
beginning of the proof,

$$
\operatorname{gr}\left(\left\{a_{1}\right\} \times\left\{x_{-k}, \mathbf{p}\right\}\right)=\operatorname{gr}\left(\{y\} \times\left\{x_{0}, \mathbf{p}\right\}\right)+2 \mathcal{F}(\mathbf{p})=\operatorname{gr}\left(\left\{x_{0}, \mathbf{p}\right\}\right)+2 \mathcal{F}(\mathbf{p}),
$$

where the distinction between $\{y\} \times\left\{x_{0}, \mathbf{p}\right\}$ and $\left\{x_{0}, \mathbf{p}\right\}$ is that the former intersection point generates in $\widehat{C F K}\left(D_{+}(K, t), 1\right)$ and the latter generates in $\widehat{C F K}(K)$ (here we treat the case $t<0$ - the case $t>0$ differs only by the addition of 1 to the right-hand side). The proof of Theorem 4.1 made use of the bijection, $B$, between generators of $\widehat{C F K}(K)=C\{i=0\}$ and $C\{\max (i, j-m)=0\}$. Restricted to $C\{i=0, j>m\}$ we have the bijection

$$
B: C\{i=0, j>m\} \rightarrow C\{i<0, j=m\} .
$$

Under $B$ the gradings satisfy,

$$
\operatorname{gr}\left(B\left(\left\{x_{0}, \mathbf{p}\right\}\right)\right)=\operatorname{gr}\left(\left\{x_{0}, \mathbf{p}\right)+2 \mathcal{F}(\mathbf{p}),\right.
$$

By the discussion in [27, Section 3]. Thus, the grading of $\left\{a_{1}\right\} \times\left\{x_{-k}, \mathbf{p}\right\}$ in $C\{i<$ $0, j=m\}$ is given by:

$$
\begin{aligned}
& \operatorname{gr}\left(\left\{a_{1}\right\} \times\left\{x_{-k}, \mathbf{p}\right\}\right)=\operatorname{gr}\left(\left\{x_{0}, \mathbf{p}\right\}\right)+ 2 \mathcal{F}(\mathbf{p})= \\
& \operatorname{gr}\left(B\left(\left\{x_{0}, \mathbf{p}\right\}\right)\right)-2 \mathcal{F}(\mathbf{p})+2 \mathcal{F}(\mathbf{p})=\operatorname{gr}\left(B\left(\left\{x_{0}, \mathbf{p}\right\}\right),\right.
\end{aligned}
$$

ie the grading of $\left[\left\{a_{1}\right\} \times\left\{x_{-k}, \mathbf{p}\right\}\right] \in \widehat{H F K}\left(D_{+}(K, t), 1\right)$ is the same as the grading it inherits as a homology class in $H_{*}(C\{i<0, j=m\})$.

Finally, we relied on the existence of a chain homotopy equivalence:

$$
\Psi: C_{*}\{i<0, j=m\} \rightarrow \mathcal{F}_{*-2 m}(K,-m-1) .
$$

This is the chain homotopy equivalence found in [27, Proposition 3.8], used to identify the Floer homology of a knot $K$, and its reverse, $-K$. The factor of $2 m$ appearing in the grading shift for $\Psi$ arises from the existence of the basepoint, $w^{\prime}$, used for the companion knot $K$. Since this basepoint is absent in hd $\left(D_{+}(K, t)\right)$, we see that the grading of $\left[\left\{a_{1}\right\} \times\left\{x_{-k}, \mathbf{p}\right\}\right] \in \widehat{H F K}\left(D_{+}(K, t), 1\right)$ is the same as the grading it inherits as a homology class in $H_{*}(\mathcal{F}(K,-m-1))$.

This takes care of the absolute gradings for generators of $\widehat{H F K}\left(D_{+}(K, t), 1\right)$ supported in the winding region. In the proof of Theorem 4.1, however, $\widehat{H F K}_{*}\left(S_{t}^{3}(K), \mu_{K}, \mathfrak{s}_{m}\right)$ was calculated for each $\operatorname{Spin}^{c}$ structure separately, each time looking only at points in the winding region. In the case of the Whitehead double all the $\operatorname{Spin}^{c}$ structures on $S_{t}^{3}(K)$ are grouped into $\widehat{H F K}\left(D_{+}(K, t), 1\right)$, and hence we must determine the absolute gradings of the generators of $\widehat{H F K}\left(D_{+}(K, t), 1\right)$ lying outside the winding region. 
To handle these generators we argue as follows: by letting $|t| \gg 0$ be sufficiently large, we can ensure that every $\operatorname{Spin}^{c}$ structure on $S_{t}^{3}(K)$ with

$$
\widehat{H F K}\left(S_{t}^{3}(K), \mu_{K}, \mathfrak{s}_{m}\right) \neq \mathbb{Z}
$$

is generated by intersection points supported in the winding region. In other words, we make the framing large enough so that the intersection points outside of the winding region only lie in $\operatorname{Spin}^{c}$ structures on $S_{t}^{3}(K)$ for which the groups $\widehat{H F K}_{*}\left(S_{t}^{3}(K), \mu_{K}, \mathfrak{s}_{m}\right)$ have stabilized (we know that these groups must eventually all be $\mathbb{Z}$, by the adjunction inequality). For these $\operatorname{Spin}^{c}$ structures, the map $\Phi_{\mathfrak{r}_{m}}$ induces an isomorphism

$$
\widehat{H F K}{ }_{*}\left(S_{t}^{3}(K), \mu_{K}, \mathfrak{s}_{m}\right) \cong \widehat{H F}\left(S^{3}\right)=\mathbb{Z} .
$$

Now since the maps $\Phi_{\mathfrak{r}_{m}}$ are invariants of the cobordism $-W_{t}^{\prime}$ and $\operatorname{Spin}^{c}$ structure $\mathfrak{r}_{m}$, it follows that we can calculate them with an arbitrary Heegaard triple diagram. Since $\Phi_{\mathfrak{r}_{m}}$ is an isomorphism for the $\mathfrak{s}_{m}$ in the stable range, it follows that there exists a pseudo-holomorphic Whitney triangle $\psi$ with $\mu(\psi)=0$ connecting the generator of $\widehat{H F K}\left(S_{t}^{3}(K), \mu_{K}, \mathfrak{s}_{m}\right)$ to the generator of $\widehat{H F}\left(S^{3}\right)$. This implies that the multiplicities of the domain of $\psi$ must all be positive and it follows that the multiplicities of $\psi$ in the domains to the right of the connect sum region must all be zero (otherwise there would be negative multiplicity somewhere in the winding region, see Figure 17). We can complete this $\psi$ to a Whitney disk $\phi$ with $\mu(\phi)=1$ as in Lemma 4.5, thus completing the proof of Theorem 4.4.

\section{Computation of $\widehat{H F K}\left(D_{+}(K, t), 1\right)$ and $\tau\left(D_{+}(K, t)\right)$ for all $t$}

In this section the skein exact sequence for knot Floer homology is used to interpolate between the case when $t \gg 0$ and the case when $t \ll 0$. This enables a calculation of $\widehat{H F K}\left(D_{+}(K, t), 1\right)$ for all values of $t$. We will also determine $\tau\left(D_{+}(K, t)\right)$. The analysis of the skein sequence will be similar to the technique used in Hedden-Ording [12] in the special case of Whitehead doubles of the $(2,2 n+1)$ torus knots. The main result of the section is the following Proposition.

Proposition 5.1 Let $K \subset S^{3}$ be a knot with Seifert genus $g(K)=g$. Then for $t \geq 2 \tau(K)$ we have:

$$
\widehat{H F K}_{*}\left(D_{+}(K, t), 1\right) \cong \mathbb{F}_{(1)}^{t-2 g-2} \bigoplus_{i=-g}^{g}\left[H_{*-1}(\mathcal{F}(K, i))\right]^{2},
$$




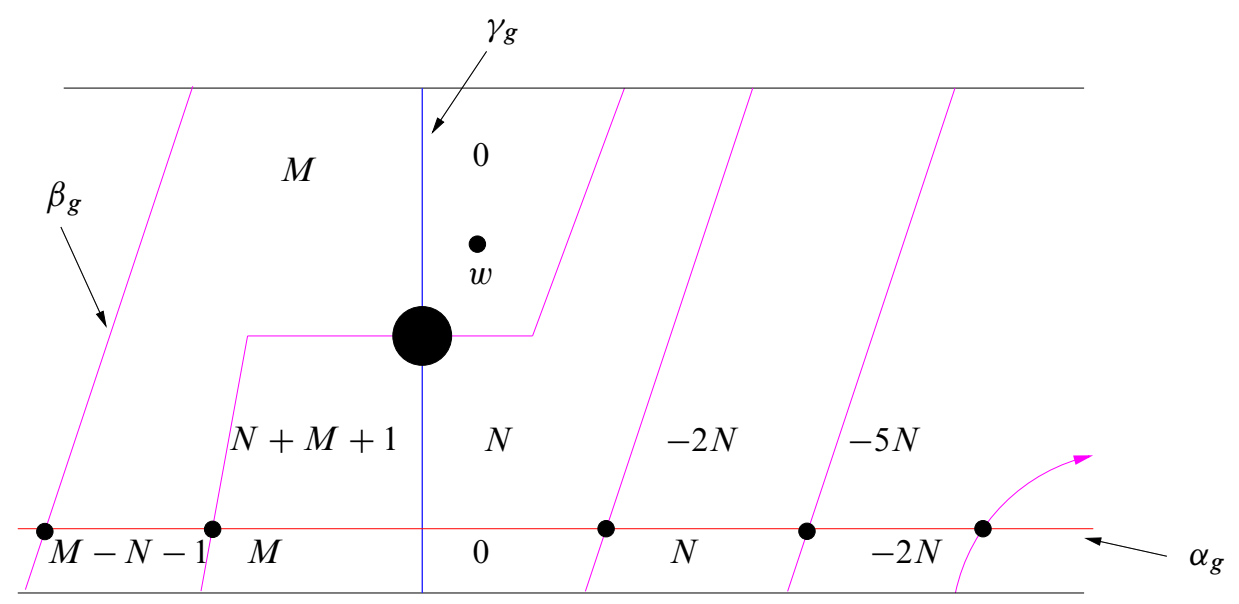

Figure 17: Illustration of the domain of an arbitrary triangle, $\psi$, connecting a generator supported away from the winding region to a generator of $\widehat{H F}\left(S^{3}\right)$. Since $n_{w}(\psi)=0$, we see that in order for $\psi$ to be holomorphic, $N=0$. Otherwise the domain of $\psi$ would have negative multiplicity. With $N=0$ we can complete $\psi$ to a Whitney disk as in Lemma 4.5 and Lemma 4.6.

and $\tau\left(D_{+}(K, t)\right)=0$. For $t<2 \tau(K)$ the following holds:

$$
\widehat{H F K}_{*}\left(D_{+}(K, t), 1\right) \cong \mathbb{F}_{(1)}^{2 \tau(K)-2 g-2} \oplus \mathbb{F}_{(0)}^{2 \tau(K)-t} \bigoplus_{i=-g}^{g}\left[H_{*-1}(\mathcal{F}(K, i))\right]^{2},
$$

and $\tau\left(D_{+}(K, t)\right)=1$.

Remark 5.2 This takes care of the top and bottom group for the Whitehead double, by the symmetry of knot Floer homology about $\mathcal{F}=0$. It also proves Theorem 1.5 stated in the introduction.

\subsection{Algebraic preliminaries}

The present and succeeding sections rely heavily on some elementary, but perhaps non-standard, homological algebra. For this reason, we find it convenient to recall some definitions and results regarding filtered chain complexes and filtered chain maps. First recall that a $\mathbb{Z}$-filtered vector space is a vector space $V$ (which for our purposes will be finite dimensional), together with an exhausting family of subspaces, $\left\{V_{i}\right\}_{i \in \mathbb{Z}}$, indexed by the integers and such that $V_{i} \subset V_{j}$ if $i \leq j$. This endows vectors in $V$ with an ordering: $v \leq v^{\prime}$ if $v^{\prime} \in V_{i}$ implies $v \in V_{i}$ for all $i$. 
A filtered linear map between filtered vector spaces, $V, W$, is a linear transformation $F: V \rightarrow W$ which respects the filtrations of $V$ and $W$ in the sense that $F\left(V_{i}\right) \subset W_{i}$ for all $i$.

Let $V$ be a filtered vector space of dimension $n$. Given a basis $\left\{e_{k}\right\}_{k=1}^{k=n}$ for $V$, a filtered change of basis is a filtered linear map, $I: V \rightarrow V$ which is invertible as a linear transformation. The collection $\left\{I\left(e_{k}\right)\right\}_{k=1}^{k=n}$ provides a new basis for $V$. Note the requirement that $I$ is filtered implies that if $e_{k} \in V_{i}$ then $I\left(e_{k}\right) \in V_{i}$. Thus a basis vector is sent to vectors with equal or lower order with respect to the filtration of $V$. By ordering any given basis compatibly with the ordering on vectors from the filtration, filtered changes of basis are equivalent to $n \times n$ invertible lower triangular matrices. (To do this, we order the basis so that $e_{i} \geq e_{j}$ if $i<j-$ thus $e_{1}$ is of highest order with respect to the filtration and $e_{n}$ is of lowest order).

A filtered chain complex is a chain complex $(C, d)$ where $C$ is a filtered (graded) vector space and the differential $d$ is a filtered linear map. Concretely, a filtered chain complex is a chain complex $C$, together with an exhausting sequence of subcomplexes:

$$
C_{1} \subset C_{2} \subset \cdots \subset C_{m}=C .
$$

The notions of a filtered chain map, filtered chain homotopy, and filtered chain homotopy equivalence are exactly the same as for ordinary chain complexes, with the added requirement that every map involved is required to be filtered linear. The results of the current and succeeding sections are about filtered chain complexes up to filtered chain homotopy equivalence. The equivalence class of a filtered chain complex $(C, d)$ up to filtered chain homotopy equivalence will be referred to as the filtered chain homotopy type of $(C, d)$. A key observation is that a filtered change of basis of $(C, d)$ does not change the filtered chain homotopy type (though it may change the appearance of the differential).

It is well known that a filtered chain complex induces a spectral sequence, and each term in this spectral sequence is itself a filtered chain complex, see McCleary [21] for an introduction. Further, $\left(E_{1}, d_{1}\right)=\left(C_{i} / C_{i-1}, d_{\text {induced }}^{i}\right)$ where $d_{\text {induced }}^{i}$ are the differentials induced on the quotient complexes $C^{i}=C_{i} / C_{i-1}$. Then the homology groups $H_{*}\left(C^{i}\right)$ generate the $E_{2}$ stage of the spectral sequence.

Given a filtered chain complex, $C$, it would often be convenient to replace $C$ with a filtered chain complex $C^{\prime}$ whose $d_{1}$ differential is trivial and which is isomorphic as a filtered graded vector space to the associated graded groups of $C$. Indeed this can be done uniquely, up to filtered chain homotopy equivalence, using a concept called the reduction of filtered chain complexes. This concept is explained in detail in Rasmussen's thesis [33, Sections 4 and 5]. More precisely we have the following lemma. 
Lemma 5.3 (Rasmussen [33, Lemma 4.5]) Let $C$ be a filtered complex with filtration

$$
C_{1} \subset C_{2} \subset \cdots \subset C_{m},
$$

and let $C^{i}=C_{i} / C_{i-1}$ be the filtered quotients, so that the homology groups $H_{*}\left(C^{i}\right)$ are the $E_{2}$ terms of the spectral sequence associated to the filtration. Then, up to filtered chain homotopy equivalence, there is a unique filtered complex $C^{\prime}$ with the following properties.

(1) $C^{\prime}$ is filtered chain homotopy equivalent to $C$.

(2) $\left(C^{\prime}\right)^{i} \cong H_{*}\left(C^{i}\right)$.

(3) The spectral sequence of the filtration on $C^{\prime}$ has trivial first differential. Its higher terms are the same as the higher terms of the spectral sequence of the filtration on $C$.

Following Rasmussen, we will refer to the chain complex $C^{\prime}$ as the reduction of $C$.

The above lemma is very useful for our purposes. It allows us to treat the knot Floer homology groups, which are the $E_{2}$ term in the spectral sequence associated to the knot filtration, as a filtered chain complex in their own right. As such, they have a differential, $d^{\prime}$, which strictly lowers the filtration (since, by Property (3) the spectral sequence of the reduction has trivial first differential). Note that while $d^{\prime}$ determines the differentials in the spectral sequence of the reduction (and hence by Property (3) the higher differentials for the original complex), in general it is not a differential in the spectral sequence for the reduction. Instead, it is the differential on a filtered chain complex which is filtered chain homotopy equivalent to the one we started with.

Recall that a filtered chain map induces a morphism of spectral sequences. The next lemma shows that this morphism is well-behaved with respect to reduction.

Lemma 5.4 (See Rasmussen [33, Lemma 5.2]) Let $C, D$ be filtered chain complexes, and $F: C \rightarrow D$ a filtered chain map. Then there is an induced filtered chain map between the reductions $F^{\prime}: C^{\prime} \rightarrow D^{\prime}$. The maps $F, F^{\prime}$ induce the same morphism of spectral sequences. $F^{\prime}$ is unique up to pre- and post-composition with filtered chain homotopy equivalences.

\subsection{Proof of Proposition 5.1}

It will be helpful to first rephrase Theorem 4.4. 
Theorem 5.5 Let $K \subset S^{3}$ be a knot, and suppose $g(K)=g$ denotes the Seifert genus of $K$. Then for all $t>T>0$ there are isomorphisms of absolutely $\mathbb{Z}$-graded abelian groups:

$$
\begin{gathered}
\widehat{H F K}_{*}\left(D_{+}(K, t), 1\right) \cong \mathbb{F}_{(1)}^{t-2 g-2} \bigoplus_{i=-g}^{g}\left[H_{*-1}(\mathcal{F}(K, i))\right]^{2} \\
\widehat{H F K}_{*}\left(D_{+}(K,-t), 1\right) \cong \mathbb{F}_{(0)}^{t-2 g} \bigoplus_{i=-g}^{g}\left[H_{*}\left(\frac{\widehat{C F}\left(S^{3}\right)}{\mathcal{F}(K, i)}\right)\right]^{2}
\end{gathered}
$$

Proof This follows from the adjunction inequality for knot Floer homology, which implies that $H_{*}(\mathcal{F}(K, i)) \cong 0$ for $i<g$ and $H_{*}(\mathcal{F}(K, i)) \cong F_{(0)}$ for $i \geq g$.

By performing $2 t$ successive crossing changes to the twisting region of the knot diagram shown in Figure 1 , we can change $D_{+}(K, t)$ into $D_{+}(K,-t)$. Each of these operations changes a negative crossing to a positive crossing. Recall that [27, Theorem 10.2] (see also Rasmussen [33]) asserts that associated to a crossing change there are skein exact sequences for knot Floer homology (for each $i$ ):

$$
\cdots \longrightarrow \widehat{H F K}\left(K_{-}, i\right) \stackrel{f_{1}}{\longrightarrow} \widehat{H F K}\left(S^{1} \times S^{2}, \kappa\left(K_{0}\right), i\right) \stackrel{f_{2}}{\longrightarrow} \widehat{H F K}\left(K_{+}, i\right) \stackrel{f_{3}}{\longrightarrow} \cdots .
$$

where the maps $f_{1}$ and $f_{2}$ lower homological grading by one-half and $f_{3}$ is nonincreasing in the homological grading. Here $K_{-}$is the knot with negative crossing, $K_{+}$is the knot with positive crossing, and $K_{0}$ is the two-component link obtained by resolving the crossing. More precisely, [27, Section 2] describes a well-defined way to associate a knot $\left(S^{1} \times S^{2}, \kappa(L)\right)$ to a two-component link $\left(S^{3}, L\right)$ and $\left(S^{1} \times\right.$ $\left.S^{2}, \kappa\left(K_{0}\right)\right)$ is this "knotification" of the link obtained from resolving the crossing.

In the case at hand, these exact sequences relate the Floer homology groups of $D_{+}(K, t)$, $D_{+}(K, t-1)$ and the two-component link obtained from the oriented resolution of the crossing which we change. Regardless of $t$ or $K$, this link is the positive Hopf link, which we denote by $H$. The knotification of $H$ is shown in Figure 3, and its Floer homology is given by the following Proposition.

Proposition 5.6 (Ozsváth-Szabó [27, Proposition 9.2])

$$
\widehat{H F K}\left(S^{1} \times S^{2}, \kappa(H), i\right) \cong \begin{cases}\mathbb{F}_{\left(\frac{1}{2}\right)} & \text { if } i=1 \\ \mathbb{F}^{2}\left(-\frac{1}{2}\right) & \text { if } i=0 \\ \mathbb{F}_{\left(-\frac{3}{2}\right)} & \text { if } i=-1 \\ 0 & \text { otherwise }\end{cases}
$$


In light of the above, we see that the exact sequence for the top filtration level takes the following form:

$$
\cdots \longrightarrow \widehat{H F K}\left(D_{+}(K, j), 1\right) \stackrel{f_{1}}{\longrightarrow} \mathbb{F}_{\left(\frac{1}{2}\right)} \stackrel{f_{2}}{\longrightarrow} \widehat{H F K}\left(D_{+}(K, j-1), 1\right) \stackrel{f_{3}}{\longrightarrow} \cdots .
$$

We wish to understand the maps in this sequence for each $j=-t+1, \ldots, t$. This is achieved through several algebraic lemmas. To simplify notation, it will be useful to define:

$$
r_{*}(t)=\operatorname{rk} \widehat{H F K}_{*}\left(D_{+}(K, t), 1\right) .
$$

Our first lemma determines how the ranks of the groups in each homological dimension differ between the cases when $t>T>0$ and $t<-T<0$.

Lemma 5.7 Let $K \subset S^{3}$ be a knot with genus $g$, and let $t>T>0$ be an integer so that Theorem 5.5 holds. Then

$$
\begin{aligned}
r_{*}(t) & =r_{*}(-t) \text { if } * \neq 0,1 \\
r_{*}(t+1) & =r_{*}(t) \text { if } * \neq 0,1 \\
r_{1}(t) & =r_{1}(-t)+t-2 \tau(K) \\
r_{0}(t) & =r_{0}(-t)-t-2 \tau(K) .
\end{aligned}
$$

Proof The lemma follows from Theorem 5.5, the definition of $\tau(K)$, and the long exact sequence in homology coming from the short exact sequence of chain complexes,

$$
0 \longrightarrow \mathcal{F}(K, j) \stackrel{i}{\longrightarrow} \widehat{C F}\left(S^{3}\right) \stackrel{p}{\longrightarrow} \frac{\widehat{C F}\left(S^{3}\right)}{\mathcal{F}(K, j)} \longrightarrow 0 .
$$

Since $\widehat{H F}\left(S^{3}\right) \cong \mathbb{F}_{(0)}$, the long exact sequence shows that

$$
H_{*-1}(\mathcal{F}(K, j)) \cong H_{*}\left(\frac{\widehat{C F}\left(S^{3}\right)}{\mathcal{F}(K, j)}\right)
$$

if $* \neq 0,1$, from which the first part of the lemma follows (taking into account the grading shift in the first part of Theorem 5.5). For the second two parts, recall that $\tau(K)$ is defined as:

$$
\tau(K)=\min \left\{j \in \mathbb{Z} \mid i_{*}: H_{*}(\mathcal{F}(K, j)) \longrightarrow \widehat{H F}\left(S^{3}\right) \text { is non-trivial }\right\} .
$$

In the long exact sequence we have:

$$
\begin{aligned}
0 \longrightarrow H_{1}\left(\frac{\widehat{C F}\left(S^{3}\right)}{\mathcal{F}(K, j)}\right) \stackrel{\delta}{\longrightarrow} H_{0}(\mathcal{F}(K, j)) \stackrel{i_{*}}{\longrightarrow} \mathbb{F}_{(0)} \stackrel{p_{*}}{\longrightarrow} \\
H_{0}\left(\frac{\widehat{C F}\left(S^{3}\right)}{\mathcal{F}(K, j)}\right) \stackrel{\delta}{\longrightarrow} H_{-1}(\mathcal{F}(K, j)) \longrightarrow 0,
\end{aligned}
$$


and the map $i_{*}$ is trivial precisely when $j<\tau(K)$ and non-trivial otherwise. Taking the sum over each $j$ from $-g, \ldots, g$, and examining ranks yields the second two parts of the lemma.

Next, we observe that the map $f_{3}$ in the Skein exact sequence, which a priori is non-increasing in the absolute degree, in fact preserves degree.

Proposition 5.8 In the exact sequence relating $\widehat{H F K}(H, 1), \widehat{H F K}\left(D_{+}(K, t), 1\right)$ and $\widehat{H F K}\left(D_{+}(K, t-1), 1\right)$, the map $f_{3}$ preserves degree.

Proof We first show that $f_{3}$ preserves degree, when restricted to $\widehat{H F K}_{*}\left(D_{+}(K, j), 1\right)$ with $* \leq 0$. Define

$$
m_{1}(t)=\min \left\{* \in \mathbb{Z} \mid *<0 \text {, and } \widehat{H F K}_{*}\left(D_{+}(K, t), 1\right) \neq 0\right\} .
$$

(This number is not well-defined if $\widehat{H F K}\left(D_{+}(K, t)\right)$ is supported in non-negative homological grading. If this is the case, proceed to the latter section of the argument.) By the preceding lemma, for all $t>T$ we see that $m_{1}(t)$ stabilizes:

$$
m_{1}(t)=m_{1}(-t):=\mathbf{m}_{1} .
$$

Further, the ranks stabilize

$$
r_{\mathbf{m}_{1}}(t)=r_{\mathbf{m}_{1}}(-t):=\mathbf{r}_{\mathbf{m}_{1}} .
$$

Begin with $-t<-T$ and the exact sequence:

$$
\cdots \longrightarrow \widehat{H F K}\left(D_{+}(K,-t+1), 1\right) \stackrel{f_{1}}{\longrightarrow} \mathbb{F}_{\left(\frac{1}{2}\right)} \stackrel{f_{2}}{\longrightarrow} \widehat{H F K}\left(D_{+}(K,-t), 1\right) \stackrel{f_{3}}{\longrightarrow} \cdots .
$$

If $f_{3}$ lowers degree when restricted to $\widehat{H F K}_{\mathbf{m}_{1}}\left(D_{+}(K,-t), 1\right)$, then $r_{*}(-t+1)>0$ for some $*<\mathbf{m}_{\mathbf{1}}$. Since $f_{3}$ is non-increasing in degree and $\mathbb{F}_{\left(\frac{1}{2}\right)}$ doesn't interact with gradings $*<0$ (by the degree shifts of $f_{1}, f_{2}$ ), we see that after $2 t$ applications of the skein sequence used to pass to $D_{+}(K, t), r_{*}(t)>0$ for some $*<\mathbf{m}_{\mathbf{1}}$. This contradicts the fact that $m_{1}(t)=\mathbf{m}_{\mathbf{1}}$. Thus $f_{3}$, restricted to $\widehat{H F K}_{\mathbf{m}_{1}}\left(D_{+}(K,-t), 1\right)$, preserves degree. Inductively, this shows that $f_{3}$ preserves degree for $*=\mathbf{m}_{1}$ for all $t$. Furthermore, it shows that $m_{1}(t)=\mathbf{m}_{1}$ for all $t$. Next define

$$
m_{2}(t)=\min \left\{* \in \mathbb{Z} \mid \mathbf{m}_{1}<*<0, \text { and } \widehat{H F K}_{*}\left(D_{+}(K, t), 1\right) \neq 0\right\} .
$$

Lemma 5.7 again shows that for all $t>T$,

$$
m_{2}(t)=m_{2}(-t):=\mathbf{m}_{2}
$$


and also that

$$
r_{*}(t)=r_{*}(-t)=\mathbf{r}_{*} \text { for all } * \leq \mathbf{m}_{\mathbf{2}} .
$$

Begin with $-t<-T$. If $f_{3}$ restricted to $\widehat{H F K}_{\mathbf{m}_{2}}\left(D_{+}(K,-t), 1\right)$ lowers degree, then $r_{*}(-t+1)>r_{*}(-t)$ for some $*<\mathbf{m}_{\mathbf{2}}$. As above, after $2 t$ applications of the skein sequence $r_{*}(t)>r_{*}(-t)$ for some $*<\mathbf{m}_{\mathbf{2}}$, contradicting Equation (8). Inductively, we see that $f_{3}$ preserves degree for $* \leq \mathbf{m}_{\mathbf{2}}$. Repeating the whole argument with $m_{3}(t), m_{4}(t), \ldots$ shows that $f_{3}$ preserves degree for homological gradings $*<0$. Moreover, if $f_{3}$ lowered degree when restricted to $*=0$, then the above argument would imply that $r_{*}(t)>r_{*}(-t)$ for some $*<0$ and $t>T$. Again, this contradicts the fact that $r_{*}(|t|)$ is independent of $t$ for large enough $|t|$ and $* \neq 0,1$. Thus $f_{3}$ preserves degree for all gradings $* \leq 0$.

Next we show that $f_{3}$ preserves degree for $*>1$. Define

$$
M_{1}(t)=\max \left\{* \in \mathbb{Z} \mid *>1 \text { and } \widehat{H F K}_{*}\left(D_{+}(K, t), 1\right) \neq 0\right\} .
$$

By Lemma 5.7, for $t>T$ we have

$$
M_{1}(t)=M_{1}(-t):=\mathbf{M}_{1}
$$

and

$$
r_{\mathbf{M}_{1}}(t)=r_{\mathbf{M}_{1}}(-t):=\mathbf{r}_{\mathbf{M}_{\mathbf{1}}} .
$$

Begin with $-t<-T$. If $f_{3}$ lowers degree when restricted to $\widehat{H F K}_{\mathbf{M}_{1}}\left(D_{+}(K,-t), 1\right)$ then $r_{\mathbf{M}_{\mathbf{1}}}(-t+1)<r_{\mathbf{M}_{\mathbf{1}}}(-t)$. Since $f_{3}$ is non-increasing in degree and $\mathbb{F}_{\left(\frac{1}{2}\right)}$ doesn't interact with gradings $*>1$, we see $r_{\mathbf{M}_{\mathbf{1}}}(t)<r_{\mathbf{M}_{\mathbf{1}}}(-t)$. This is a contradiction. Inductively, we see that $f_{3}$, restricted to $*=\mathbf{M}_{1}$, preserves degree. Then, as above, repeat with

$$
M_{i}(t)=\max \left\{* \in \mathbb{Z} \mid \mathbf{M}_{\mathbf{i}-1}>*>1, \text { and } \widehat{H F K}_{*}\left(D_{+}(K, t), 1\right) \neq 0\right\}
$$

in place of $M_{i-1}(t)$ for $i=2,3, \ldots$ to show that $f_{3}$ preserves degree for $*>1$.

Thus $f_{3}$ preserves degree provided $* \neq 1$. Suppose now that $f_{3}$ lowers degree for $*=1$. Then $f_{3}$ maps non-trivially from $*=1$ to either $*<0$ or $*=0$. In the first case, the argument above shows that $r_{*}(t)>r_{*}(-t)$ for some $*<0$, contradicting Lemma 5.7. For the second case, note that in each application of the skein sequence $f_{2}$ is either trivial or non-trivial. If $f_{2}=0$, then

$$
\left[r_{1}(j)-r_{1}(j-1)\right] \leq 1 \quad \text { and } \quad\left[r_{0}(j-1)-r_{0}(j)\right] \leq 0,
$$

while if $f_{2} \neq 0$,

$$
\left[r_{1}(j)-r_{1}(j-1)\right] \leq 0 \quad \text { and } \quad\left[r_{0}(j-1)-r_{0}(j)\right] \leq 1 .
$$


In either case, we have

$$
\left[r_{1}(j)-r_{1}(j-1)\right]+\left[r_{0}(j-1)-r_{0}(j)\right] \leq 1,
$$

with equality if and only if $f_{3}$ preserves degree for $*=1$ (these inequalities implicitly use the knowledge that $f_{3}$ preserves degree for all $* \neq 1$ ). Taking the sum,

$$
\begin{aligned}
\Sigma_{j=-t+1}^{j=t}\left\{\left[r_{1}(j)-r_{1}(j-1)\right]+\left[r_{0}(j-1)-r_{0}(j)\right]\right\} & = \\
& {\left[r_{1}(t)-r_{1}(-t)\right]+\left[r_{0}(-t)-r_{0}(t)\right] \leq 2 t, }
\end{aligned}
$$

with equality if and only if $f_{3}$ preserves degree for $*=1$, and for all $j$. However, Lemma 5.7 shows that

$$
\left[r_{1}(t)-r_{1}(-t)\right]+\left[r_{0}(-t)-r_{0}(t)\right]=2 t,
$$

completing the proof.

Lemma 5.9 In the $2 t$ applications of the skein sequence connecting $D_{+}(K, t)$ and $D_{+}(K,-t)$, the map

$$
f_{2}: \widehat{H F K}(H, 1) \cong \mathbb{F}_{\left(\frac{1}{2}\right)} \rightarrow \widehat{H F K}\left(D_{+}(K, j-1), 1\right),
$$

is trivial exactly $t-2 \tau(K)$ times.

Proof Proposition 5.8 indicates that $f_{3}$ preserves degree, so $\widehat{H F K}\left(D_{+}(K, j-1), 1\right)$ is determined by $\widehat{H F K}\left(D_{+}(K, j), 1\right)$ and whether or not $f_{2}$ is trivial:

$$
\begin{array}{ll}
\widehat{H F K}\left(D_{+}(K, j-1), 1\right) \cong \widehat{H F K}\left(D_{+}(K, j), 1\right) / \mathbb{F}_{(1)} & \text { if } f_{2}=0 \\
\widehat{H F K}\left(D_{+}(K, j-1), 1\right) \cong \widehat{H F K}\left(D_{+}(K, j), 1\right) \oplus \mathbb{F}_{(0)} & \text { if } f_{2} \neq 0
\end{array}
$$

In particular, we have

$$
\begin{aligned}
r_{1}(j)=r_{1}(j-1)+1 & \text { if } f_{2}=0 \\
r_{1}(j)=r_{1}(j-1) & \text { if } f_{2} \neq 0 .
\end{aligned}
$$

Thus, on the one hand

$$
\Sigma_{j=-t+1}^{j=t}\left[r_{1}(j)-r_{1}(j-1)\right]=r_{1}(t)-r_{1}(-t)=\#\left\{\text { times } f_{2} \text { is trivial }\right\} .
$$

While on the other hand, Lemma 5.7 shows that for $t>T>0$ and $-t<-T<0$,

$$
r_{1}(t)-r_{1}(-t)=t-2 \tau(K) .
$$

Next, recall Hedden-Ording [12, Proposition 2.4]. 
Proposition 5.10 In the exact sequence for filtration grading 1 above, $f_{2} \neq 0$ if and only if $\tau\left(D_{+}(K, t-1)\right)=1$. Otherwise $\tau\left(D_{+}(K, t-1)\right)=0$.

Proof The proposition will follow from the fact that $f_{2}$ is the lowest order term in a filtered chain map, $\tilde{f}_{2}$, between filtered chain complexes which are chain homotopy equivalent to $\widehat{C F}\left(S^{1} \times S^{2}\right)$ and $\widehat{C F}\left(S^{3}\right)$, respectively.

To begin, recall from Lemma 5.3 that the Floer homology groups for $H$ (respectively, $\left.D_{+}(K, t-1)\right)$ can be endowed with a differential, $d^{\prime}$, which gives them the structure of a filtered chain complex. Moreover, $d^{\prime}$ strictly lowers the filtration index, and the filtered chain homotopy type of $\left(\widehat{H F K}(H), d^{\prime}\right)\left(\operatorname{resp} .\left(\widehat{H F K}\left(D_{+}(K, t-1)\right), d^{\prime}\right)\right)$ is the same as that of $(\widehat{C F K}(H), d)$ (resp. $\left.\left(\widehat{C F K}\left(D_{+}(K, t-1)\right), d\right)\right)$. In the language of Section 5.1, $\left(\widehat{H F K}(H), d^{\prime}\right)$ and $\left(\widehat{H F K}\left(D_{+}(K, t-1)\right), d^{\prime}\right)$ are the reductions of the filtered chain complexes $(\widehat{C F K}(H), d)$ and $\left(\widehat{C F K}\left(D_{+}(K, t-1)\right), d\right)$ coming from the Heegaard diagrams. Since the knot Floer homology of $H$ is the Floer homology for the knotification, $\kappa(H) \subset S^{1} \times S^{2}$, we have

$$
H_{*}\left(\widehat{H F K}(H), d^{\prime}\right) \cong \widehat{H F}\left(S^{1} \times S^{2}\right) \cong \mathbb{F}_{\left(-\frac{1}{2}\right)} \oplus \mathbb{F}_{\left(\frac{1}{2}\right)} .
$$

In the case of $D_{+}(K, t-1)$, we have

$$
H_{*}\left(\widehat{H F K}\left(D_{+}(K, t-1)\right), d^{\prime}\right) \cong \widehat{H F}\left(S^{3}\right) \cong \mathbb{F}_{(0)} .
$$

The filtration on the knot Floer homology of $D_{+}(K, t-1)$ induces a filtration grading on $\widehat{H F}\left(S^{3}\right)$ in the standard way, ie the filtration of any cycle, $z=\Sigma n_{x} x$, is by definition the maximum filtration of any chain $x$ which comprises $z$. Now $\tau\left(D_{+}(K, t-1)\right)$ can be equivalently defined as the minimum filtration grading of any cycle $z \in \widehat{H F K}\left(D_{+}(K, t-\right.$ 1)) which is homologous to a generator of $\widehat{H F}\left(S^{3}\right)$.

The proof of the skein sequence [27, Theorem 8.2] relies, in part, on the fact that there is a filtered chain map

$$
\tilde{f}_{2}:(\widehat{C F K}(H), d) \rightarrow\left(\widehat{C F K}\left(D_{+}(K, t-1)\right), d\right) .
$$

This filtered chain map induces maps on the $E_{2}$ terms of the spectral sequence (ie on $\widehat{H F K}(K, i))$, and these are the $f_{2}$ maps in the skein sequence. Lemma 5.4 indicates that there is an induced filtered chain map between the reduction complexes:

$$
\tilde{f}_{2}^{\prime}:\left(\widehat{H F K}(H), d^{\prime}\right) \rightarrow\left(\widehat{H F K}\left(D_{+}(K, t-1)\right), d^{\prime}\right),
$$

and further that $\tilde{f}_{2}^{\prime}$ induces the same morphism of spectral sequences as $\tilde{f}_{2}$, provided we start with the $E_{2}$ stage of the spectral sequences for $\widehat{C F K}$. From this point on, we work exclusively with the reduced complexes and with $\tilde{f}_{2}^{\prime}$. We can do this since working with the reduced objects is the same as working with the original chain 
complexes and chain maps, up to filtered chain homotopy equivalence (by Lemma 5.3 and Lemma 5.4).

The discussion above indicates that $\tilde{f}_{2}^{\prime}$ decomposes as a sum of homogeneous pieces, each of which lower the filtration by some fixed integer. Further, the $f_{2}$ map in question is the part of ${\widetilde{f_{2}^{\prime}}}_{2}$ which (a) preserves the filtration and (b) has domain $\widehat{H F K}(H, 1)$.

It follows from Proposition 5.6 that the unique chain generating $\widehat{H F K}(H, 1) \cong \mathbb{F}_{\left(\frac{1}{2}\right)}$ is a cycle under $d^{\prime}$, indeed $\mathrm{rk} \widehat{H F}_{\frac{1}{2}}\left(S^{1} \times S^{2}\right)=1$ and $\operatorname{rk} \widehat{H F K} \widehat{1}_{\frac{1}{2}}(H, i)=0$ unless $i=1$. Since $\widetilde{f_{2}^{\prime}}$ is a chain map, it must map this chain to a cycle $z$ in $\widehat{H F K}\left(D_{+}(K, t-1)\right)$. Now if $f_{2} \neq 0, z$ contains non-trivial chains with filtration index 1 . Since $\widehat{H F K}\left(D_{+}(K, t-\right.$ $1), i) \cong 0$ for $i>1, z$ cannot be the boundary of any chain. This is because in the reduced complex there are no chains in filtration levels with $\widehat{H F K}\left(D_{+}(K, t-1), i\right)=0$. Thus $z$ is a generator for $\widehat{H F}\left(S^{3}\right)$ and contains non-trivial chains with filtration index 1 . If $z^{\prime}$ were a cycle homologous to $z$ which only contained chains of filtration index $-1,0$, then there would have to exist a chain $w$, such that $d^{\prime}(w)=z+z^{\prime}$. However, any such chain must have filtration greater than 1 , since $d^{\prime}$ strictly lowers filtration index. This contradicts the fact that $\widehat{H F K}\left(D_{+}(K, t-1), i\right)=0$ for $i>1$. Thus, the filtration grading of $z$-which is 1 - is the minimum over all chains homologous to the generator of $\widehat{H F}\left(S^{3}\right)$ ie $\tau\left(D_{+}(K, t-1)\right)=1$.

Having shown that $f_{2} \neq 0$ implies $\tau\left(D_{+}(K, t-1)\right)=1$, we wish to show that $\tau\left(D_{+}(K, t-1)\right)=1$ implies $f_{2} \neq 0$. To show this, observe that since $\tilde{f}_{2}{ }^{\prime}$ is a chain map between the reductions, it induces a map on homology:

$$
\widehat{H F}\left(S^{1} \times S^{2}\right) \cong \mathbb{F}_{\left(-\frac{1}{2}\right)} \oplus \mathbb{F}_{\left(\frac{1}{2}\right)} \stackrel{\left(\tilde{f}_{2}^{\prime}\right)_{*}}{\longrightarrow} \widehat{H F}\left(S^{3}\right) \cong \mathbb{F}_{(0)} .
$$

and this map sends the space supported in grading $\frac{1}{2}$ to the generator. If $\tau(D(t-1))=1$, any cycle representing the generator of $\widehat{H F}\left(S^{3}\right)$ contains non-trivial chains in filtration level 1. Since the grading one-half space of $\widehat{H F}\left(S^{1} \times S^{2}\right)$ is supported entirely in filtration level equal to 1 , it follows that $f_{2}$ - the part of $\tilde{f}_{2}^{\prime}$ which preserves the filtration - maps non-trivially from $\widehat{H F K}(H, 1)$ to $\widehat{H F K}\left(D_{+}(K, t-1), 1\right)$.

Finally, if $\tau\left(D_{+}(K, t)\right)=-1$ for some $t$, then any cycle generating $\widehat{H F}\left(S^{3}\right)$ is supported entirely in $\widehat{H F K}\left(D_{+}(K, t),-1\right)$. As above, there is a chain map

$$
\widetilde{f}_{1}^{\prime}:\left(\widehat{H F K}\left(D_{+}(K, t)\right), d^{\prime}\right) \rightarrow\left(\widehat{H F K}(H), d^{\prime}\right),
$$

whose filtration-preserving pieces induce the $f_{1}$ maps in the skein sequence. Further, the induced map on homology

$$
\widehat{H F}\left(S^{3}\right) \cong \mathbb{F}_{(0)} \stackrel{\left(\tilde{f}_{1}^{\prime}\right)_{*}}{\longrightarrow} \widehat{H F}\left(S^{1} \times S^{2}\right) \cong \mathbb{F}_{\left(-\frac{1}{2}\right)} \oplus \mathbb{F}_{\left(\frac{1}{2}\right)}
$$


sends a generator of $\widehat{H F}\left(S^{3}\right)$ to the space supported in grading $-\frac{1}{2}$. However, this latter space is supported entirely in filtration level zero by Proposition 5.6, and thus $\widetilde{f}_{1}^{\prime}$ must raise the filtration grading, contradicting the fact that it is a filtered chain map. $\square$

To complete the proof of Proposition 5.1, note that Equations (9) and (10) indicate that $\widehat{H F K}\left(D_{+}(K, j-1), 1\right)$ is determined by $\widehat{H F K}\left(D_{+}(K, j), 1\right)$ and whether $f_{2} \neq 0$. Further, the previous proposition shows that $\tau\left(D_{+}(K, j-1)\right)$ is also determined by whether $f_{2} \neq 0$. Thus it remains to understand the behavior of $f_{2}$. Indeed, Lemma 5.9 showed that in the $2 t$ applications of the skein sequence used to pass from $D_{+}(K, t)$ to $D_{+}(K,-t), f_{2}=0$ exactly $t-2 \tau(K)$ times - hence we need only determine the $j$ for which $f_{2}=0$. Proposition 5.1 will follow if we can show that $f_{2}=0$ for $j=t, t-1, \ldots, 2 \tau(K)+1$.

To this end, recall that Livingston [18] and Ozsváth-Szabó [26] prove that $\tau(K)$ satisfies the following inequality under the operation of changing a crossing in a projection of $K$ :

$$
\tau\left(K_{+}\right)-1 \leq \tau\left(K_{-}\right) \leq \tau\left(K_{+}\right)
$$

Since each application of the skein sequence arose from changing a single negative crossing to a positive crossing, the above inequality becomes (for $k>0$ ):

$$
\tau\left(D_{+}(K, j-k)\right)-k \leq \tau\left(D_{+}(K, j)\right) \leq \tau\left(D_{+}(K, j-k)\right) .
$$

If $f_{2}$ were non-trivial for some $j$ and trivial for $j-k$, then Proposition 5.10 would imply $\tau\left(D_{+}(K, j-1)\right)=1$ and $\tau\left(D_{+}(K, j-k-1)\right)=0$, violating the inequality. Thus $f_{2}=0$ for the first $t-2 \tau(K)$ applications of the skein sequence and $f_{2} \neq 0$ thereafter ie $f_{2}=0$ for $j=t, t-1, \ldots, 2 \tau(K)+1$. This completes the proof of Proposition 5.1.

\section{Computation of $\widehat{H F K}\left(D_{+}(K, t), 0\right)$ and higher differen- tials}

In this section, we complete the calculation of the filtered chain homotopy type of $\widehat{H F K}\left(D_{+}(K, t)\right)$. Throughout the discussion, we will be making use of Lemma 5.3 which allows us to replace the knot Floer homology chain complex $(\widehat{C F K}(K), d)$ coming from the Heegaard diagram with a filtered chain complex $\left(\widehat{H F K}(K), d^{\prime}\right)$ called the reduction of $(\widehat{C F K}(K), d)$. The key properties we will use of the reduction complex are as follows.

(1) It is filtered chain homotopy equivalent to $(\widehat{C F K}(K), d)$. 
(2) Its chain groups are isomorphic, as filtered graded vector spaces, to the knot Floer homology groups, ie to the associated graded groups of $(\widehat{C F K}(K), d)$.

(3) The $d_{0}^{\prime}$ differential is trivial for $\left(\widehat{H F K}(K), d^{\prime}\right)$ ie the associate graded groups of $\left(\widehat{H F K}(K), d^{\prime}\right)$ are the same as the $E_{0}$ groups.

Thus we replace the knot Floer homology chain complex with a filtered chain complex whose generators (chains) are in one-to-one correspondence with a basis for the knot Floer homology groups (which in the present case are filtered graded vector spaces since we use field coefficients).

In the present situation, the adjunction inequality [27, Theorem 5.1] implies that $\widehat{H F K}\left(D_{+}(K, t), i\right) \cong 0$ when $|i|>1$, since $D_{+}(K, t)$ has a genus one Seifert surface. Hence Lemma 5.3 shows that the groups $\widehat{H F K}\left(D_{+}(K, t)\right)$ can be viewed as a filtered chain complex whose differential is composed of three distinct homomorphisms:

$$
\begin{aligned}
& d_{2}: \widehat{H F K}_{*}\left(D_{+}(K, t), 1\right) \longrightarrow \widehat{H F K}_{*-1}\left(D_{+}(K, t),-1\right) \\
& d_{1}^{i}: \widehat{H F K}_{*}\left(D_{+}(K, t), i\right) \longrightarrow \widehat{H F K}_{*-1}\left(D_{+}(K, t), i-1\right), \quad i=1,0 .
\end{aligned}
$$

Furthermore, the maps $d_{1}^{i}$ are induced by chain maps

$$
\partial_{1}^{i}: \widehat{C F K}_{*}\left(D_{+}(K, t), i\right) \longrightarrow \widehat{C F K}_{*-1}\left(D_{+}(K, t), i-1\right),
$$

defined by counting holomorphic disks which satisfy $n_{z}(\phi)=1, n_{w}(\phi)=0$. Under the differential $d^{\prime}=d_{2}+d_{1}^{1}+d_{1}^{0}$, the homology of $\left(\widehat{H F K}\left(D_{+}(K, t)\right), d^{\prime}\right)$ is isomorphic to $\widehat{H F}\left(S^{3}\right) \cong \mathbb{F}_{(0)}$.

The following is a useful algebraic lemma for the case at hand:

Lemma 6.1 Suppose $g(K)=1$ and $\tau(K) \neq-1$. Then the following are equivalent up to filtered chain homotopy equivalence:

(1) $d_{2}=0$

(2) $d_{1}^{0}$ is surjective.

Proof To see that (2) implies (1), assume that $d_{1}^{0}$ is surjective and that $\operatorname{dim}(\operatorname{Im}$ $\left.d_{2}\right)>0$. Thus, there exists a one-dimensional vector subspace, $V$, of $\widehat{H F K}(K, 1)$ mapped isomorphically to a one-dimensional subspace of $\widehat{H F K}(K,-1)$. Let $a$ be a generator of $V$ and $c$ be a generator of its image, $c=d_{2}(a)$. Since $d_{1}^{0}$ is assumed to be surjective, let $b$ be a generator of a subspace $V^{\prime} \subset \widehat{H F K}(K, 0)$ such that $d_{1}^{0}(b)=c$. There are now two possibilities:

(1) $d_{1}^{1}(a)=0$, 
(2) $d_{1}^{1}(a)=d \neq 0$.

(See Figure 18 for an illustration). We claim that in both cases, the filtered subcomplex consisting of the generators $a, b, c$ (resp. $a, b, c, d$ ) is filtered chain homotopy equivalent to a chain complex for which $d_{2}=0$. To see this, recall from Section 5.1 that a filtered change of basis of a filtered vector space is a change of basis which maps each basis element to a sum of basis elements of equal or lower order with respect to the filtration. If a filtered vector space, $C$, has the structure of a filtered chain complex (ie there exists a morphism, $d: C \rightarrow C$ such that $\mathcal{F}(d(x)) \leq \mathcal{F}(x)$ and such that $d \circ d=0$ ) then any filtered change of basis is a filtered chain homotopy equivalence between the original chain complex and the "new" chain complex with alternative basis. Indeed both the change of basis transformation and its inverse are chain maps, and the fact that the inverse map is is a filtered chain map (ie respects the filtration) follows from the fact that the inverse of a lower diagonal matrix is lower diagonal. Their composite is clearly filtered chain homotopic, in fact equal, to the identity. Thus, for Case (1), we use the change of basis:

$$
\begin{aligned}
& a \rightarrow a^{\prime}=a+b \\
& b \rightarrow b^{\prime}=b \\
& c \rightarrow c^{\prime}=c,
\end{aligned}
$$

while for Case (2) we use the same basis change and simply let $d \rightarrow d^{\prime}=d$. In each case, the differential in the new basis satisfies $d_{2}(a)=0$. See Figure 18 for an illustration. Proceeding inductively, we arrive at a basis for which $d_{2}=0$.

To see that (1) implies (2) assume that $d_{2}=0$. The fact that $\tau(K) \neq-1$ implies that any generator of $\widehat{H F}\left(S^{3}\right)$ contains chains in either the middle or top filtration level. This further implies that all the chains in $\widehat{H F K}(K,-1)$ are the boundary of chains in either $\widehat{H F K}(K, 0)$ or $\widehat{H F K}(K, 1)$ - if not, some cycle in $\widehat{H F K}(K,-1)$ would generate a non-trivial class in $\widehat{H F}\left(S^{3}\right)$. Since, $d_{2}=0$, chains in $\widehat{H F K}(K,-1)$ can only be the boundary of chains in $\widehat{H F K}(K, 0)$ under $d_{1}^{0}$, and hence $d_{1}^{0}$ is surjective. Thus the lemma is proved.

If we can show that $d_{1}^{0}$ is surjective, then Theorem 1.2 will follow from Lemma 6.1 and the following proposition holds.

Proposition 6.2 Suppose $g(K)=1$ and $\tau(K)=0$ (resp. $\tau(K)=1$ ). Further, suppose $d_{2}=0$. Then

$$
\begin{aligned}
\widehat{H F K}_{*}(K, 0) & \cong \mathbb{F}_{(0)} \oplus \widehat{H F K}_{*+1}(K, 1) \oplus \widehat{H F K}_{*-1}(K,-1) \\
\quad(\text { resp. } & \left.\cong \widehat{H F K}_{*+1}(K, 1) \oplus \widehat{H F K_{*-1}}(K,-1) / \mathbb{F}_{(0)}\right)
\end{aligned}
$$



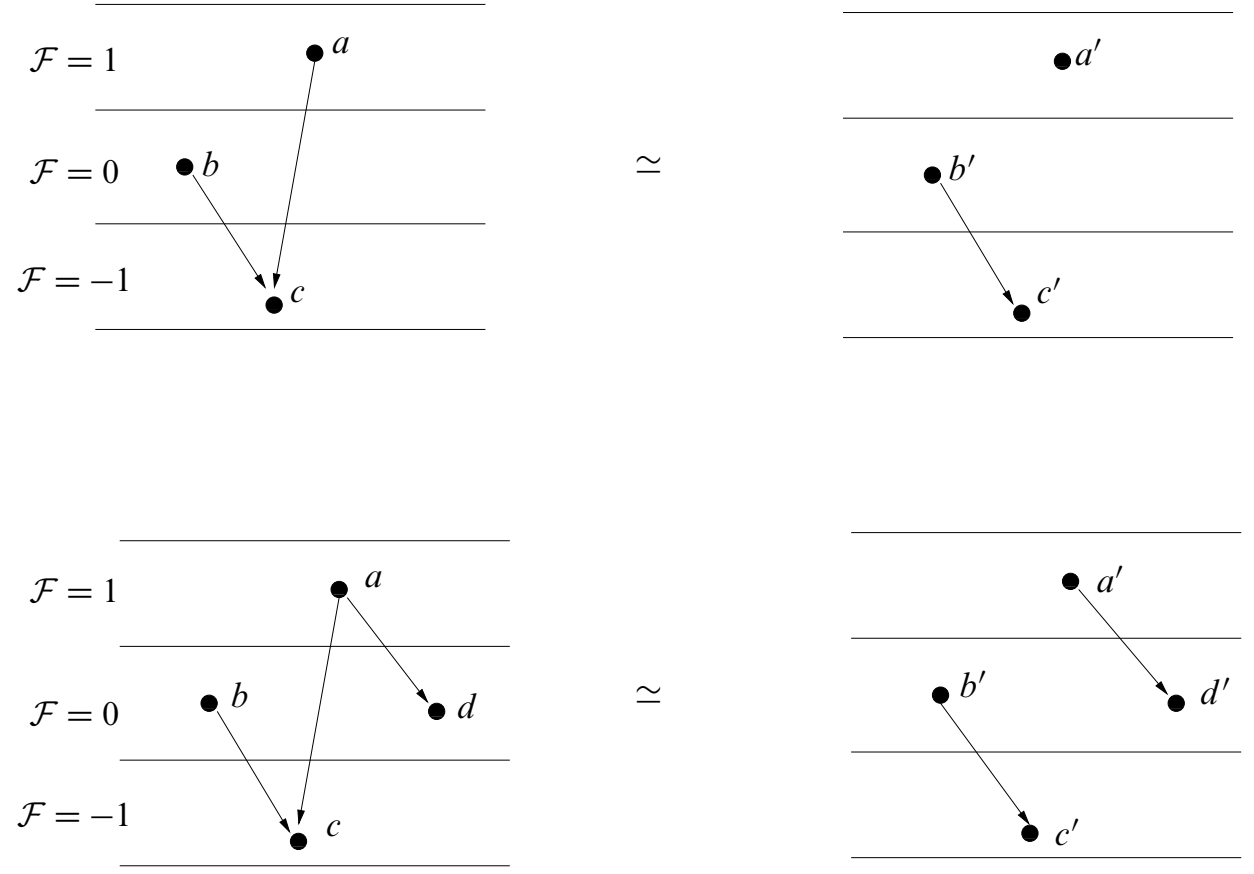

Figure 18: Illustration of filtered chain homotopy equivalent chain complexes discussed in the proof of Lemma 6.1. A dot represents a basis element for a one-dimensional vector space (a chain), and an arrow represents an isomorphism between one-dimensional vector spaces (a non-trivial differential). The filtration of chains is indicated by vertical height and the index $\mathcal{F}=i, i=1,0,-1$. The chain complexes differ by the filtered change of basis described in the text.

Remark 6.3 To avoid confusion, we note that in the above formula the $\mathbb{F}_{(0)}$ summand only occurs when $*=0$.

Proof The proposition follows from the fact that that the homology of $\left(\widehat{H F K}(K), d^{\prime}\right)$ is isomorphic to $\mathbb{F}_{(0)}$, with $\tau(K)$ equal to the filtration grading of the generator of this homology. Suppose that $\tau(K)=0$. Then none of the generators in $\widehat{H F K}_{*}(K, 1)$ can be cycles (since $\widehat{H F}\left(S^{3}\right)$ is one-dimensional). Further, since $d_{2}=0, d_{1}^{1}$ must map $\widehat{H F K}_{*}(K, 1)$ injectively into a summand $V \subset \widehat{H F K}_{*-1}(K, 0)$ isomorphic to $\widehat{H F K}(K, 1)$. Similarly, since $\tau(K) \neq-1$, every generator of $\widehat{H F K} *(K,-1)$ must be the boundary of a chain in $\widehat{H F K}_{*+1}(K, 0)$ or $\widehat{H F K}_{*+1}(K, 1)$. Since $d_{2}=0$, $\widehat{H F K}_{*}(K,-1)$ must be the image of a summand $W \subset \widehat{H F K} \widehat{H+1}_{*}(K, 0)$ isomorphic to $\widehat{H F K}(K,-1)$. The case for $\tau(K)=1$ follows from similar considerations. 
Hence it remains to show that $d_{1}^{0}$ is surjective. Recall from Section 3, that the chain complex for the middle filtration level took the following form:

$$
\widehat{C F K}\left(D_{+}(K, t), 0\right) \sim\left[\left\{a_{2}\right\} \times \widehat{C F}\left(S_{t}^{3}(K)\right)\right] \oplus\left[\left\{a_{4}\right\} \times \widehat{C F}\left(S_{t}^{3}(K)\right)\right] \oplus[\{y\} \times \widehat{C F K}(K)] .
$$

We first prove the following Proposition.

Proposition 6.4 Under the splitting of the generators of $\widehat{C F K}\left(D_{+}(K, t), 0\right)$ given above, the generators $\left[\left\{a_{2}\right\} \times \widehat{C F}\left(S_{t}^{3}(K)\right)\right]$ form a subcomplex.

Proof We must show that the boundary of any generator of the form $\left\{a_{2}\right\} \times \mathbf{p}$ consists of generators of the same form. Assume otherwise, that there exists a holomorphic Whitney disk, $\phi$ connecting $\left\{a_{2}\right\} \times \mathbf{p}$ to a generator of the form $\left\{a_{4}\right\} \times \mathbf{q}$ or $\{y\} \times \mathbf{q}^{\prime}$ satisfying $n_{z}(\phi)=n_{w}(\phi)=0$. In order for $\phi$ to satisfy $n_{z}(\phi)=n_{w}(\phi)=0$ and simultaneously be a disk oriented from $\left\{a_{2}\right\} \times \mathbf{q}$, it must have negative multiplicity in one or both of the regions illustrated in Figure 19. This contradicts the fact that $\phi$ is holomorphic.

Since $\left[\left\{a_{2}\right\} \times \widehat{C F}\left(S_{t}^{3}(K)\right)\right]$ is a subcomplex, the restriction of the chain map $\partial_{1}^{0}$ will be a chain map:

$$
\left.\left(\partial_{1}^{0}\right)\right|_{\left[\left\{a_{2}\right\} \times \widehat{C F}\left(S_{t}^{3}(K)\right)\right]}:\left[\left\{a_{2}\right\} \times \widehat{C F}\left(S_{t}^{3}(K)\right)\right] \longrightarrow \widehat{C F K}\left(D_{+}(K, t),-1\right) .
$$

Proposition 6.5 The restriction of $\partial_{1}^{0}$ to $\left[\left\{a_{2}\right\} \times \widehat{C F}\left(S_{t}^{3}(K)\right)\right]$ induces an isomorphism on homology.

Proof There is a canonical "small" Whitney disk connecting $\left\{a_{2}\right\} \times \mathbf{p}$ to $\left\{a_{3}\right\} \times \mathbf{p}$ for any $(g+1)$-tuple, $\mathbf{p}$, and which satisfies $n_{z}(\phi)=1, n_{w}(\phi)=0$. See Figure 9 . The domain of this disk is topologically an annulus, and can be seen to admit a unique holomorphic representative for a suitably generic choice of almost complex structure on $\operatorname{Sym}^{g+2}\left(\Sigma_{g+2}\right)$, see $[29 ; 28]$. In the standard way (see $\left.[27 ; 28]\right)$, we can filter the chain map $\partial_{1}^{0}$ with respect to negative area of domains of disks. With respect to this filtration, we have:

$$
\left.\left(\partial_{1}^{0}\right)\right|_{\left[\left\{a_{2}\right\} \times \widehat{C F}\left(S_{t}^{3}(K)\right)\right]}=I+\Phi,
$$

where $I$ is an isomorphism induced by the aforementioned small disks and $\operatorname{Im}(\Phi)$ consists of terms with lower order filtration. Hence $\left.\left(\partial_{1}^{0}\right)\right|_{\left[\left\{a_{2}\right\} \times \widehat{C F}\left(S_{t}^{3}(K)\right)\right]}$ induces an isomorphism on homology. 


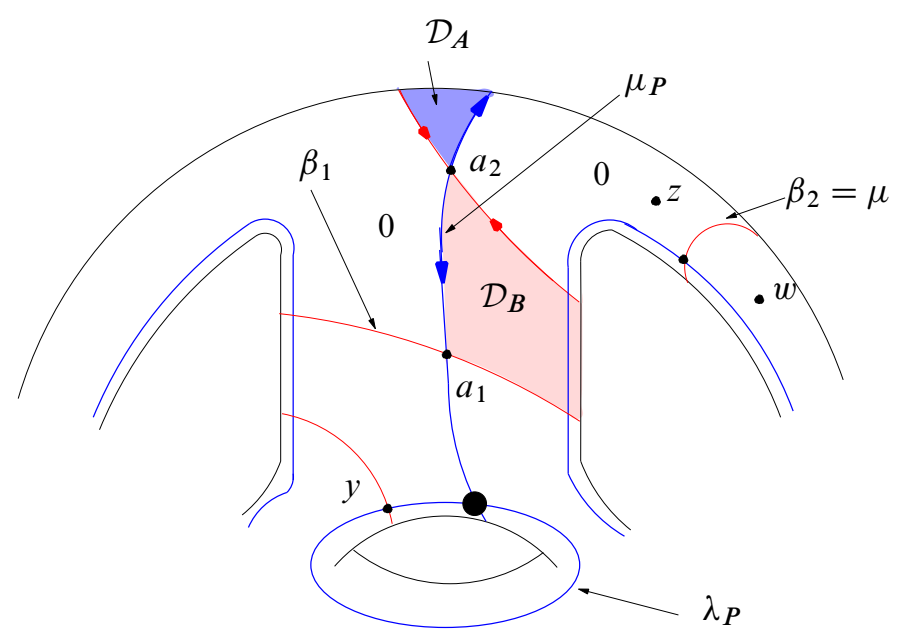

Figure 19: Depiction of possible domains of Whitney disks, $\phi$, connecting a generator $\left\{a_{2}\right\} \times \mathbf{p}$ to generators $\left\{a_{4}\right\} \times \mathbf{q}$ or $\{y\} \times \mathbf{q}^{\prime}$, restricted to the Heegaard surface for the pattern. Since $\phi$ is oriented to go from $a_{2}$, the boundary of the domain of $\phi$ must be oriented as shown by the arrows in the figure. The requirement that $\phi$ satisfies $n_{z}(\phi)=n_{w}(\phi)=0$ implies the domain of $\phi$ must have non-zero multiplicity in one, or both, of the domains, $\mathcal{D}_{A}, \mathcal{D}_{B}$. The orientation of the boundary of $\phi$ and the inward normal orientation of the Heegaard surface imply the multiplicity of $\phi$ in $\mathcal{D}_{A}$ or $\mathcal{D}_{B}$ is negative.

This completes our proof of Theorem 1.2: the restriction of $\partial_{1}^{0}$ to $\left[\left\{a_{2}\right\} \times \widehat{C F}\left(S_{t}^{3}(K)\right)\right]$ can be factored as $\partial_{1}^{0} \circ i$, where $i$ is the inclusion:

$$
i:\left[\left\{a_{2}\right\} \times \widehat{C F}\left(S_{t}^{3}(K)\right)\right] \longrightarrow \widehat{C F K}\left(D_{+}(K, t), 0\right) .
$$

Since the restriction induces an isomorphism on homology, the map induced by $\partial_{1}^{0}$, ie $d_{1}^{0}$, is surjective.

\section{Preliminary Applications}

We conclude with two simple applications of Theorem 1.2 and some qualitative remarks.

\subsection{Iterated Doubles of the figure eight knot}

We have the following closed formula for the Floer homology of the iterated untwisted Whitehead doubles of the figure eight knot. 
Proposition 7.1 Let $4_{1}$ be the figure eight knot and let $D^{n}$ denote the $n$th iterated untwisted double of $4_{1}$ ie $D^{0}=4_{1}, D^{n}=D_{+}\left(D^{n-1}, 0\right)$ Then we have:

$$
\widehat{H F K}_{*}\left(D^{n}, i\right) \cong \begin{cases}\bigoplus_{k=0}^{n} \mathbb{F}_{(1-k)}^{2^{n}\left(\begin{array}{c}
n \\
k
\end{array}\right)} & i=1 \\
\mathbb{F}_{(0)} \bigoplus_{k=0}^{n} \mathbb{F}_{(-k)}^{2^{n+1}\left(\begin{array}{l}
n \\
k
\end{array}\right)} & i=0 \\
\bigoplus_{k=0}^{n} \mathbb{F}_{(-1-k)}^{2^{n}\left(\begin{array}{l}
n \\
k
\end{array}\right)} & i=-1 \\
0 & \text { otherwise. }\end{cases}
$$

Furthermore, the induced differential $d_{1}^{1}$ is injective, $d_{1}^{0}$ is surjective, and $d_{2}$ is zero. Hence $\tau\left(D^{n}\right)=0$.

Proof In order to apply Theorem 1.2 iteratively, we use our knowledge of the induced differentials $d_{1}^{1}, d_{1}^{0}, d_{2}$ acting on $\widehat{H F K}\left(D_{+}(K, t)\right)$ which was determined in the preceding section. We proceed by induction on $n$. For $n=0$, we have $D^{0}=4_{1}=D_{+}(U, 1)$. $\widehat{H F K}\left(4_{1}\right)$ can be determined using various methods (see, for example $[33 ; 25]$ ) but we choose to use Theorem 1.2. Recall that the unknot has Floer homology

$$
\widehat{H F K}_{*}(U, 0) \cong \mathbb{F}_{(0)}
$$

and that $\widehat{H F K}_{*}(U, i) \cong 0$ for $i \neq 0$. This immediately implies that $H_{*}(\mathcal{F}(U, 0)) \cong \mathbb{F}_{(0)}$ and that $\tau(U)=0$. Hence we see that $\bigoplus_{i=-0}^{0}\left[H_{*}(\mathcal{F}(U, i))\right] \cong F_{(0)}$. Plugging this result into the formula of Theorem 1.2 for $\widehat{H F K}\left(D_{+}(U, 1)\right.$ ) (where we use the parameters $t=1, \tau(U)=0$, and $g(U)=0$ ), we see that

$$
\widehat{H F K}_{*}\left(D_{+}(U, 1), i\right) \cong \begin{cases}\mathbb{F}_{(1)}^{-1} \oplus \mathbb{F}_{(1)}^{2}=\mathbb{F}_{(1)} & i=1 \\ \mathbb{F}_{(0)}^{-1} \oplus \mathbb{F}_{(0)}^{4}=\mathbb{F}_{(0)}^{3} & i=0 \\ \mathbb{F}_{(-1)}^{-1} \oplus \mathbb{F}_{(-1)}^{2}=\mathbb{F}_{(-1)} & i=-1 \\ 0 & \text { otherwise. }\end{cases}
$$

Furthermore, we know from the previous section and the fact that we are in the case when $t=1>2 \tau(U)$ that $d_{1}^{1}$ is injective, $d_{1}^{0}$ is surjective, and $d_{2}$ is zero. This completes the base case.

Assume that the proposition holds for $n$. This implies that

$$
\left.H_{*}\left(\mathcal{F}\left(D^{n},-1\right)\right)\right) \cong \widehat{H F K}\left(D^{n},-1\right) \cong \bigoplus_{k=0}^{n} \mathbb{F}_{(-1-k)}^{2^{n}\left(\begin{array}{c}
n \\
k
\end{array}\right)}
$$


As for $\left.H_{*}\left(\mathcal{F}\left(D^{n}, 0\right)\right)\right)$ we have,

$$
\begin{aligned}
\left.H_{*}\left(\mathcal{F}\left(D^{n}, 0\right)\right)\right) & \cong H_{*}\left(\widehat{H F K}\left(D^{n},-1\right) \oplus \widehat{H F K}\left(D^{n}, 0\right), d_{1}^{0}\right) \\
& \cong H_{*}\left(\bigoplus_{k=0}^{n} \mathbb{F}_{(-1-k)}^{2^{n}\left(\begin{array}{l}
n \\
k
\end{array}\right)} \oplus \mathbb{F}_{(0)} \bigoplus_{k=0}^{n} \mathbb{F}_{(-k)}^{\left.2^{n+1}\left(\begin{array}{c}
n \\
k
\end{array}\right), d_{1}^{0}\right)}\right. \\
& \cong \mathbb{F}_{(0)} \bigoplus_{k=0}^{n} \mathbb{F}_{(-k)}^{2^{n}\left(\begin{array}{l}
n \\
k
\end{array}\right)},
\end{aligned}
$$

where the first congruence is the definition of $\mathcal{F}(K, 0)$ for a genus one knot, the second follows from our inductive hypothesis, and the final follows from the fact that $d_{1}^{0}$ is assumed to be surjective.

For the final filtration, we clearly have $H_{*}\left(\mathcal{F}\left(D^{n}, 1\right)\right) \cong \mathbb{F}_{(0)}$. Thus, we see that

$$
\bigoplus_{i=-1}^{1}\left[H_{*}\left(\mathcal{F}\left(D^{n}, i\right)\right)\right] \cong\left[\bigoplus_{k=0}^{n} \mathbb{F}_{(-1-k)}^{2^{n}\left(\begin{array}{c}
n \\
k
\end{array}\right)}\right] \oplus\left[\mathbb{F}_{(0)} \bigoplus_{k=0}^{n} \mathbb{F}_{(-k)}^{2^{n}\left(\begin{array}{c}
n \\
k
\end{array}\right)}\right] \oplus\left[F_{(0)}\right]=\mathbb{F}_{(0)}^{2} \bigoplus_{k=0}^{n+1} \mathbb{F}_{(-k)}^{2^{n}\left(\begin{array}{c}
n+1 \\
k
\end{array}\right)}
$$

Applying Theorem 1.2 with parameters $t=0, \tau\left(D^{n}\right)=0, g\left(D^{n}\right)=1$, we have that:

$$
\widehat{H F K}_{*}\left(D^{n+1}, i\right) \cong\left\{\begin{array}{cl}
\mathbb{F}_{(1)}^{-4} \bigoplus_{i=-1}^{1}\left[H_{*-1}\left(\mathcal{F}\left(D^{n}, i\right)\right)\right]^{2} & \\
=\mathbb{F}_{(1)}^{-4} \oplus \mathbb{F}_{(1)}^{4} \bigoplus_{k=0}^{n+1} \mathbb{F}_{(-k)}^{2^{n+1}\left(\begin{array}{c}
n+1 \\
k
\end{array}\right)} & i=1 \\
\mathbb{F}_{(0)}^{-7} \bigoplus_{i=-1}^{1}\left[H_{*}\left(\mathcal{F}\left(D^{n}, i\right)\right)\right]^{4} & \\
=\mathbb{F}_{(0)}^{-7} \oplus \mathbb{F}_{(0)}^{8} \bigoplus_{k=0}^{n+1} \mathbb{F}_{(-k)}^{2^{n+2}\left(\begin{array}{c}
n+1 \\
k
\end{array}\right)} & i=0 \\
\mathbb{F}_{(-1)}^{-4} \bigoplus_{i=-1}^{1}\left[H_{*+1}\left(\mathcal{F}\left(D^{n}, i\right)\right)\right]^{2} & \\
=\mathbb{F}_{(-1)}^{-4} \oplus \mathbb{F}_{(-1)}^{4} \bigoplus_{k=0}^{n+1} \mathbb{F}_{(-k)}^{2^{n+1}\left(\begin{array}{c}
n+1 \\
k
\end{array}\right)} & i=-1 . \\
0 & \text { otherwise. }
\end{array}\right.
$$

Quotienting by the negative exponents in the above equation yields the formula given by Proposition 7.1 for $D^{n+1}$, thus completing the inductive step. Theorem 1.2 and Lemma 6.1 show that $d_{1}^{1}$ is injective, $d_{1}^{0}$ is surjective and $d_{2}=0$.

We found it notable that while the figure eight knot is an alternating knot and has particularly simple Floer homology, by forming its iterated untwisted doubles we obtain knot Floer homology groups which become incredibly complicated. In particular, the width of the Floer homology (the number of diagonals on which knot Floer homology is supported, plotted on a grid whose axes are the homological and filtration grading) can be made arbitrarily large. Indeed, the width grows linearly with the number of times we double. Also, the total rank of the Floer homology grows exponentially with the number of times we double. 


\subsection{Surgery on Whitehead doubles and gluing knot complements}

As a final application, we determine the Floer homology of +1 -surgery on the Whitehead double of a knot, $K$. As mentioned in the introduction, some of these manifolds can also be seen to arise from a gluing of knot complements. In particular, if we let $T$ denote the right-handed trefoil, then +1 surgery on $D_{+}(K,-1)$ can alternately be obtained from $S^{3}-K$ and $S^{3}-T$ by identifying the boundary tori through the identification:

$$
\mu_{K} \leftrightarrow \lambda_{T} \quad \lambda_{K} \leftrightarrow \mu_{T}
$$

For a proof of this, we refer the interested reader to Auckly [3, Figure 6].

Proposition 7.2 Let $S_{+1}^{3}\left(D_{+}(K, t)\right)$ denote the manifold obtained by +1 -surgery on $D_{+}(K, t)$. Then for $t \geq 2 \tau(K)$ we have:

$$
\widehat{H F}_{*}\left(S_{+1}^{3}\left(D_{+}(K, t)\right)\right) \cong\left(\mathbb{F}_{(-1)}^{t-2 g-2} \oplus \mathbb{F}_{(0)}^{t-2 g-1} \bigoplus_{i=-g}^{g}\left[H_{*+1}(\mathcal{F}(K, i))\right]^{2} \bigoplus_{i=-g}^{g}\left[H_{*}(\mathcal{F}(K, i))\right]^{2} .\right.
$$

While for $t<2 \tau(K)$ we have:

$$
\begin{aligned}
\widehat{H F}_{*}\left(S_{+1}^{3}\left(D_{+}(K, t)\right)\right) & \cong \mathbb{F}_{(-1)}^{4 \tau(K)-t-2 g-2} \oplus \mathbb{F}_{(-2)}^{2 \tau(K)-t} \\
& \oplus \mathbb{F}_{(0)}^{2 \tau(K)-2 g-2} \bigoplus_{i=-g}^{g}\left[H_{*+1}(\mathcal{F}(K, i))\right]^{2} \bigoplus_{i=-g}^{g}\left[H_{*}(\mathcal{F}(K, i))\right]^{2} .
\end{aligned}
$$

Proof This is a straightforward application of [27, Theorem 4.4], together with Theorem 1.2. For a genus one knot, $K$, [27, Theorem 4.4] identifies

$$
\widehat{H F}_{*}\left(S_{+1}^{3}(K)\right) \cong H_{*}(C\{\max (i, j)=0\}) .
$$

And this latter group is equal to:

$$
H_{*}\left(\widehat{H F K}_{*}(K, 1)\{-2\} \oplus \widehat{H F K}_{*}(K, 0) \oplus \widehat{H F K}_{*}(K,-1), d_{1}^{0}+\widetilde{d}_{1}^{0}\right),
$$

where $d_{1}^{0}: \widehat{H F K}_{*}(K, 0) \rightarrow \widehat{H F K}_{*-1}(K,-1)$ is the map induced by the chain map $\partial_{1}^{0}$ discussed in Section 6. The map $\widetilde{d}_{1}^{0}: \widehat{H F K}_{*}(K, 0) \rightarrow \widehat{H F K}_{*+1}(K, 1)$ is induced by the chain map $\widetilde{\partial}_{1}^{0}: \widehat{H F K}_{*}(K, 0) \rightarrow \widehat{H F K}_{*+1}(K, 1)$ which counts pseudoholomorphic Whitney disks satisfying $n_{w}(\phi)=1, n_{z}(\phi)=0$. The $\{-2\}$ indicates that we shift the grading of $\widehat{H F K}_{*}(K, 1)$ down by 2 (this is induced by the action of $U$ on $\left.C F K^{\infty}(K)\right)$. In the present situation, Theorem 1.2 informs us of the groups $\widehat{H F K}_{*}\left(D_{+}(K, t), 1\right)\{-2\} \oplus \widehat{H F K} \widehat{H}_{*}\left(D_{+}(K, t), 0\right) \oplus \widehat{H F K_{*}}\left(D_{+}(K, t),-1\right)$, and furthermore that $d_{1}^{0}$ is surjective. It follows from algebraic properties of $C F K^{\infty}(K)$ that 
$\tilde{d}_{1}^{0}$ will also be surjective. Alternatively, this can be seen by the same method used in Section 6 to show that $d_{1}^{0}$ is surjective. The proposition follows.

\section{References}

[1] S Akbulut, R Matveyev, Exotic structures and adjunction inequality, Turkish J. Math. 21 (1997) 47-53 MR1456158

[2] S Akbulut, R Matveyev, A convex decomposition theorem for 4-manifolds, Internat. Math. Res. Notices (1998) 371-381 MR1623402

[3] D Auckly, Surgery numbers of 3-manifolds: a hyperbolic example, from: "Geometric topology (Athens, GA, 1993)", AMS/IP Stud. Adv. Math. 2, Amer. Math. Soc., Providence, RI (1997) 21-34 MR1470719

[4] T D Cochran, R E Gompf, Applications of Donaldson's theorems to classical knot concordance, homology 3-spheres and property P, Topology 27 (1988) 495-512 MR976591

[5] E Eftekhary, Filtration of Heegaard Floer homology and gluing formulas (2004) arXiv:math/0410356

[6] E Eftekhary, Longitude Floer homology and the Whitehead double, Algebr. Geom. Topol. 5 (2005) 1389-1418 MR2171814

[7] M H Freedman, A geometric reformulation of 4-dimensional surgery, Topology Appl. 24 (1986) 133-141 MR872483

[8] M H Freedman, F Quinn, Topology of 4-manifolds, Princeton Mathematical Series 39, Princeton University Press, Princeton, NJ (1990) MR1201584

[9] R E Gompf, A I Stipsicz, 4-manifolds and Kirby calculus, Graduate Studies in Mathematics 20, American Mathematical Society, Providence, RI (1999) MR1707327

[10] M Hedden, On knot Floer homology and cabling, Algebr. Geom. Topol. 5 (2005) 1197-1222 MR2171808

[11] M Hedden, On knot Floer homology and cabling, $\mathrm{PhD}$ thesis, Columbia University (2005) Available at http://math.mit.edu/ mhedden/thesis.ps

[12] M Hedden, P Ording, The Ozsváth-Szabó and Rasmussen concordance invariants are not equal to appear, Amer. J. Math.

[13] M Khovanov, A categorification of the Jones polynomial, Duke Math. J. 101 (2000) 359-426 MR1740682

[14] R Kirby, Problems in Low-Dimensional Topology (1995)

[15] W B R Lickorish, An introduction to knot theory, Graduate Texts in Mathematics 175, Springer, New York (1997) MR1472978 
[16] R Lipshitz, A cylindrical reformulation of Heegaard Floer homology, Geom. Topol. 10 (2006) 955-1097 MR2240908

[17] R Lipshitz, Heegaard-Floer invariants of bordered 3-manifolds, $\mathrm{PhD}$ thesis, Stanford University (2006)

[18] C Livingston, Computations of the Ozsváth-Szabó knot concordance invariant, Geom. Topol. 8 (2004) 735-742 MR2057779

[19] C Livingston, S Naik, Ozsváth-Szabó and Rasmussen invariants of doubled knots, Algebr. Geom. Topol. 6 (2006) 651-657 MR2240910

[20] C Manolescu, B Owens, A concordance invariant from the Floer homology of double branched covers (2005) arXiv:math.GT/0508065

[21] J McCleary, User's guide to spectral sequences, Mathematics Lecture Series 12, Publish or Perish, Wilmington, DE (1985) MR820463

[22] Y Ni, Sutured Heegaard diagrams for knots, Algebr. Geom. Topol. 6 (2006) 513-537 MR2220687

[23] P Ording, The knot Floer homology of satellite $(1,1)$ knots, PhD thesis, Columbia University (2006)

[24] PS Ozsváth, Z Szabó, Holomorphic triangles and invariants for smooth four-manifolds (2001) arXiv:math.SG/0110169

[25] P Ozsváth, Z Szabó, Heegaard Floer homology and alternating knots, Geom. Topol. 7 (2003) 225-254 MR1988285

[26] P Ozsváth, Z Szabó, Knot Floer homology and the four-ball genus, Geom. Topol. 7 (2003) 615-639 MR2026543

[27] P Ozsváth, Z Szabó, Holomorphic disks and knot invariants, Adv. Math. 186 (2004) 58-116 MR2065507

[28] P Ozsváth, Z Szabó, Holomorphic disks and three-manifold invariants: properties and applications, Ann. of Math. (2) 159 (2004) 1159-1245 MR2113020

[29] P Ozsváth, Z Szabó, Holomorphic disks and topological invariants for closed threemanifolds, Ann. of Math. (2) 159 (2004) 1027-1158 MR2113019

[30] P Ozsváth, Z Szabó, Knot Floer homology and integer surgeries (2005) arXiv: math.GT/0410300

[31] P Ozsváth, Z Szabó, Knot Floer homology and rational surgeries (2005) arXiv: math.GT/0504404

[32] J Rasmussen, Floer homology of surgeries on two-bridge knots, Algebr. Geom. Topol. 2 (2002) 757-789 MR1928176

[33] J Rasmussen, Floer homology and knot complements, $\mathrm{PhD}$ thesis, Harvard University (2003) 
[34] J Rasmussen, Khovanov homology and the slice genus (2004) arXiv: math.GT/0402131

[35] L Rudolph, Quasipositivity as an obstruction to sliceness, Bull. Amer. Math. Soc. (N.S.) 29 (1993) 51-59 MR1193540

Department of Mathematics, Massachusetts Institute of Technology,

77 Massachusetts Avenue, Cambridge MA 02139-4307, USA

mhedden@math.mit .edu

http://math.mit.edu/ mhedden

Proposed: Ron Fintushel

Received: 12 October 2006

Seconded: Peter Teichner, Ron Stern

Accepted: 20 August 2007 